JOURNAL OF THE

AMERICAN MATHEMATICAL SOCIETY

Volume 24, Number 2, April 2011, Pages 411-469

S 0894-0347(2010)00689-3

Article electronically published on December 28, 2010

\title{
THE SATO-TATE CONJECTURE FOR HILBERT MODULAR FORMS
}

\author{
THOMAS BARNET-LAMB, TOBY GEE, AND DAVID GERAGHTY
}

\section{CONTEnTs}

1. Introduction 411

2. Notation 415

3. An automorphy lifting theorem 415

4. A character-building exercise 436

5. Twisting and untwisting 450

6. Potential automorphy in weight $0 \quad 454$

7. Hilbert modular forms 457

Acknowledgments $\quad 467$

References

\section{INTRODUCTION}

In this paper we prove the Sato-Tate conjecture for Hilbert modular forms. More precisely, we prove the natural generalisation of the Sato-Tate conjecture for regular algebraic cuspidal automorphic representations of $\mathrm{GL}_{2}\left(\mathbb{A}_{F}\right), F$ a totally real field, which are not of CM type.

Several special cases of this result were proved in the last few years. The papers HSBT10 and Tay08 prove the result for elliptic curves over totally real fields which have potentially multiplicative reduction at some place, and it is straightforward to extend this result to the case of cuspidal automorphic representations of weight 0 (i.e., those corresponding to Hilbert modular forms of parallel weight 2) which are a twist of the Steinberg representation at some finite place. The case of modular forms (over $\mathbb{Q}$ ) of weight 3 whose corresponding automorphic representations are a twist of the Steinberg representation at some finite place was treated in Gee09, via an argument that depends on the existence of infinitely many ordinary places. The case of modular forms (again over $\mathbb{Q}$ ) was proved in [BLGHT09] (with no assumption on the existence of a Steinberg place). The main new features of the arguments of [BLGHT09] were the use of an idea of Harris ([Har09]) to ensure that potential automorphy need only be proved in weight 0 , together with a new potential automorphy theorem for $n$-dimensional Galois representations which are

Received by the editors December 17, 2009 and, in revised form, November 4, 2010.

2010 Mathematics Subject Classification. Primary 11F33.

The second author was partially supported by NSF grant DMS-0841491.

(C)2010 American Mathematical Society Reverts to public domain 28 years from publication 
symmetric powers of those attached to non-ordinary modular forms. Recent developments in the theory of the trace formula remove the need for an assumption of the existence of a Steinberg place in both this theorem and in the case of elliptic curves over totally real fields.

In summary, the Sato-Tate conjecture has been proved for modular forms and for elliptic curves over totally real fields, but is not known in any non-trivial case for Hilbert modular forms not of parallel weight 2 over any field other than $\mathbb{Q}$. It seems to be hard to extend the arguments of either [Gee09] or [BLGHT09] to the general case; in the former case, one has no way of establishing the existence of infinitely many ordinary places (although it is conjectured that the set of such places should be of density one), and in the latter case, one has no control over the mixture of ordinary and supersingular places over any rational prime. In this paper, we adopt a new approach: we combine the approach of Gee09, which is based on taking congruences to representations of $\mathrm{GL}_{2}\left(\mathbb{A}_{F}\right)$ of weight 0 , with the twisting argument of [Har09] (or rather the version of this argument used in [BLGHT09]). These techniques do not in themselves suffice to prove the result, as one has to prove an automorphy lifting theorem for non-ordinary representations over a ramified base field. No such theorems are known for representations of dimension greater than two. The chief innovation of this paper is a new technique for proving such results.

Our new automorphy lifting theorem uses the usual Taylor-Wiles-Kisin patching techniques, but rather than identifying an entire deformation ring with a Hecke algebra, we prove that certain global Galois representations, whose restrictions to decomposition groups lie on certain components of the local lifting rings, are automorphic. That this is the "natural" output of the Taylor-Wiles-Kisin method is at least implicit in the work of Kisin; cf. Section 2.3 of [Kis07. One has to be somewhat careful in making this precise, because it is necessary to use fixed lattices in the global Galois representations one considers, and to work with lifting rings rather than deformation rings. In particular, it is not clear that the set of irreducible components of a local lifting ring containing a particular $\mathcal{O}_{K}$-valued point, $K$ a finite extension of $\mathbb{Q}_{l}$, is determined by the equivalence class of the corresponding $K$-representation. This necessitates a good deal of care to work with $\mathcal{O}_{K}$-liftings throughout the paper.

Effectively (modulo the remarks about lattices in the previous paragraph) the automorphy lifting theorem that we prove tells us that if we are given two congruent $n$-dimensional $l$-adic regular crystalline essentially self-dual representations of $G_{F}$ (the absolute Galois group of a totally real field $F$ ) with the same $l$-adic Hodge types, with "the same ramification properties", and satisfying a standard assumption on the size of the mod $l$ image, then if one of them is automorphic, so is the other. By "the same ramification properties", we mean that they are ramified at the same set of places, and that the points determined by the two representations on the corresponding local lifting rings lie on the same components. For example, we require that the two representations have unipotent ramification at exactly the same set of places; we do not know how to adapt Taylor's techniques for avoiding Ihara's lemma ([Tay08]) to this more general setting.

The local deformation rings for places not dividing $l$ are reasonably well-understood, so that it is possible to verify that this condition holds at such places in concrete examples. On the other hand, the components of the crystalline deformation rings of fixed weight are not at all understood if $l$ ramifies in $F$, unless $n=2$ 
and the representations are Barsotti-Tate, when there are at most two components, corresponding to ordinary and non-ordinary representations. This might appear to prevent us from being able to apply our theorem to any representations at all. We get around this problem by making use of the few cases where the components are known. Specifically, we use the cases where $n=2$ and either the representations are Barsotti-Tate; or $F$ is unramified in $l$, and the representations are crystalline of low weight. To explain how we are able to bootstrap from these two cases, we now explain the main argument.

We begin with a regular algebraic cuspidal representation $\pi$ of $\mathrm{GL}_{2}\left(\mathbb{A}_{F}\right)$, assumed not to be of CM type. By a standard analytic argument, it suffices to prove that for each $n \geq 1$ the $(n-1)$-st symmetric power of $\pi$ is potentially automorphic, in the sense that there is a finite Galois extension $F^{\prime \prime} / F$ of totally real fields and an automorphic representation $\pi_{n}$ of $\mathrm{GL}_{n}\left(\mathbb{A}_{F^{\prime \prime}}\right)$ whose $L$-function is equal to that of the base change to $F^{\prime \prime}$ of the $(n-1)$-st symmetric power $L$-function of $\pi$. Equivalently, if we fix a prime $l$, then it suffices to prove that the $(n-1)$-st symmetric power of an $l$-adic Galois representation corresponding to $\pi$ is potentially automorphic, i.e. that its restriction to $G_{F^{\prime \prime}}$ is automorphic. This is what we prove.

We choose $l$ to be large and to split completely in $F$, and such that $\pi_{v}$ is unramified at all places $v$ of $F$ lying over $l$. We begin by making a preliminary solvable base change to a totally real field $F^{\prime} / F$, such that the base change $\pi_{F^{\prime}}$ of $\pi$ to $F^{\prime}$ is either unramified or an unramified twist of the Steinberg representation at each finite place of $F^{\prime}$. We then choose an automorphic representation $\pi^{\prime}$ of $\mathrm{GL}_{2}\left(\mathbb{A}_{F^{\prime}}\right)$ of weight 0 which is congruent to $\pi$, which for any place $v \nmid l$ is unramified (respectively an unramified twist of the Steinberg representation) if and only if $\pi$ is, and which is a principal series representation (possibly ramified) or a supercuspidal representation for all $v \mid l$. Furthermore we choose $\pi^{\prime}$ so that for places $v \mid l, \pi_{v}$ is ordinary if and only if $\pi_{v}^{\prime}$ is ordinary.

We now prove that the $(n-1)$-st symmetric power of $\pi^{\prime}$ is potentially automorphic over some finite Galois extension $F^{\prime \prime}$ of $F$. This is straightforward, although it is not quite in the literature. This is the only place that we need to make use of a potential automorphy theorem for an $n$-dimensional Galois representation, and the theorems of HSBT10 (or rather the versions of them which are now available thanks to improvements in our knowledge of the trace formula, which remove the need for discrete series hypotheses) would suffice, but for the convenience of the reader we use a theorem from [BLGHT09] (which, for instance, already includes the improvements made possible by our enhanced understanding of the trace formula) instead. This also allows us to avoid having to make an argument with Rankin-Selberg convolutions as in HSBT10. We note that the theorem we use from BLGHT09 is for ordinary representations, rather than the far more technical result for supersingular representations that is also proved in BLGHT09.

We now wish to deduce the potential automorphy of the $(n-1)$-st symmetric power of $\pi$, or rather the automorphy of the corresponding $l$-adic Galois representation $r: G_{F^{\prime \prime}} \rightarrow \mathrm{GL}_{n}\left(\overline{\mathbb{Q}}_{l}\right)$, from the automorphy of the $l$-adic Galois representation $r^{\prime}: G_{F^{\prime \prime}} \rightarrow \mathrm{GL}_{n}\left(\overline{\mathbb{Q}}_{l}\right)$ corresponding to the $(n-1)$-st symmetric power of $\pi^{\prime}$. We cannot directly apply our automorphy lifting theorem, because the Hodge-Tate weights of $r^{\prime}$ and $r$ are different. Instead, we employ an argument of Harris ([Har09]), and tensor both $r$ and $r^{\prime}$ with representations obtained by the automorphic induction of algebraic characters of a certain CM field. The choice of the field and the characters 
is somewhat delicate, in order to preserve various technical assumptions for the automorphy lifting theorem, in particular the assumption of a big residual image. The two characters are chosen so that the resulting Galois representations $r^{\prime \prime}$ and $r^{\prime \prime \prime}$ are potentially crystalline with the same Hodge-Tate weights. The representation $r^{\prime \prime \prime}$ is automorphic, by standard results on automorphic induction. We then apply our automorphy lifting theorem to deduce the automorphy of $r^{\prime \prime}$. The automorphy of $r$ then follows by an argument as in Har09 (although we employ a version of this which is very similar to that used in BLGHT09).

In order to apply our automorphy lifting theorem, we need to check the local hypotheses. At places not dividing $l$, these essentially follow from the construction of $\pi^{\prime}$, together with a path-connectedness argument, and a check (using the Ramanujan conjecture) that a certain point on a local lifting ring is smooth. At the places dividing $l$ the argument is rather more involved. At the places where $\pi$ is ordinary the hypothesis can be verified (after a suitable base change) using the results of [Ger09]. At the non-ordinary places we proceed more indirectly. For each non-ordinary $v \mid l$ we choose two local 2-dimensional $l$-adic representations $\rho$ and $\rho^{\prime}$ of $G_{F_{v}}$ which are induced from characters of quadratic extensions. The representations $\rho$ and $\rho^{\prime}$ are chosen to be congruent to the local Galois representations attached to $\pi, \pi^{\prime}$ respectively, with $\rho$ crystalline of the same Hodge-Tate weights as the local representation attached to $\pi$, and $\rho^{\prime}$ non-ordinary and potentially Barsotti-Tate. Then $\rho$ is on the same component of the local crystalline lifting ring as the local representation attached to $\pi$, and a similar statement is true for $\rho^{\prime}$ and $\pi^{\prime}$ after a base change to make it crystalline (using the knowledge of the components of Barsotti-Tate lifting rings mentioned above). Since the image of an irreducible component under a continuous map is irreducible, a straightforward argument shows that we need only check that the Galois representations corresponding to the $(n-1)$-st symmetric powers of $\rho$ and $\rho^{\prime}$, when tensored with the Galois representations obtained from the characters induced from the CM field, lie on a common component of a crystalline deformation ring (possibly after a base change). We ensure this by choosing our characters so that the two Galois representations are both direct sums of unramified twists of the same crystalline characters, and making a path-connectedness argument.

We should note that we have suppressed some technical details in the above outline of our argument; we need to take considerable care to ensure that the hypotheses relating to residual Galois representations having a big image are satisfied. In addition, as mentioned above, rather than working with Galois representations valued in fields it is essential to work with fixed lattices throughout. We remark that in the forthcoming paper BLGGT10] we remove the need to consider lattices, and generalise the arguments of this paper to prove potential automorphy theorems for a broad class of Galois representations.

We now outline the structure of the paper. In Section 2 we recall some basic notation and definitions from previous papers on automorphy lifting theorems. The automorphy lifting theorem is proved in Section 3, together with some results on the behaviour of local lifting rings under conjugation and functorialities. The most technical section of the paper is Section 4, where we construct the characters of $\mathrm{CM}$ fields that we need in the main argument. In Section 5 we recall various standard results on base change and automorphic induction and give an exposition of Harris's trick in the level of generality we require. In Section 6 we prove a 
potential automorphy theorem in weight 0 ; the precise result we require is not in the literature, and while it is presumably clear to the experts how to prove it, we provide the details. Finally, in Section 7 we carry out the strategy described above and deduce the Sato-Tate conjecture.

\section{Notation}

If $M$ is a field, we let $G_{M}$ denote its absolute Galois group. We write all matrix transposes on the left; so ${ }^{t} A$ is the transpose of $A$. Let $\epsilon$ denote the $l$-adic or mod $l$ cyclotomic character. If $M$ is a finite extension of $\mathbb{Q}_{p}$ for some $p$, we write $I_{M}$ for the inertia subgroup of $G_{M}$. If $R$ is a local ring we write $\mathfrak{m}_{R}$ for the maximal ideal of $R$.

We fix an algebraic closure $\overline{\mathbb{Q}}$ of $\mathbb{Q}$ and regard all algebraic extensions of $\mathbb{Q}$ as subfields of $\overline{\mathbb{Q}}$. For each prime $p$ we fix an algebraic closure $\overline{\mathbb{Q}}_{p}$ of $\mathbb{Q}_{p}$ and we fix an embedding $\overline{\mathbb{Q}}_{\mathbb{Q}_{p}}$. In this way, if $v$ is a finite place of a number field $F$, we have a homomorphism $G_{F_{v}} \hookrightarrow G_{F}$.

We will use some of the notation and definitions of [CHT08] without comment. In particular, we will use the notions of RACSDC (regular, algebraic, conjugate self-dual, cuspidal) and RAESDC (regular, algebraic, essentially self-dual, cuspidal) automorphic representations, for which see Sections 4.2 and 4.3 of [CHT08]. We will also use the notion of a RAECSDC (regular, algebraic, essentially conjugate selfdual, cuspidal) automorphic representation, for which see Section 1 of [BLGHT09]. If $\pi$ is a RAESDC automorphic representation of $\mathrm{GL}_{n}\left(\mathbb{A}_{F}\right), F$ a totally real field,

and $\iota: \overline{\mathbb{Q}}_{l} \stackrel{\sim}{\longrightarrow} \mathbb{C}$, then we let $r_{l, \iota}(\pi): G_{F} \rightarrow \mathrm{GL}_{n}\left(\overline{\mathbb{Q}}_{l}\right)$ denote the corresponding Galois representation. Similarly, if $\pi$ is a RAECSDC or RACSDC automorphic representation of $\mathrm{GL}_{n}\left(\mathbb{A}_{F}\right), F$ a $\mathrm{CM}$ field (in this paper, all CM fields are totally imaginary), and $\iota: \overline{\mathbb{Q}}_{l} \stackrel{\sim}{\longrightarrow} \mathbb{C}$, then we let $r_{l, \iota}(\pi): G_{F} \rightarrow \mathrm{GL}_{n}\left(\overline{\mathbb{Q}}_{l}\right)$ denote the corresponding Galois representation. For the properties of $r_{l, \iota}(\pi)$, see Theorems 1.1 and 1.2 of [BLGHT09]. If $K$ is a finite extension of $\mathbb{Q}_{p}$ for some $p$, we will let $\operatorname{rec}_{K}$ be the local Langlands correspondence of [HT01, so that if $\pi$ is an irreducible complex admissible representation of $\mathrm{GL}_{n}(K)$, then $\operatorname{rec}_{K}(\pi)$ is a Weil-Deligne representation of the Weil group $W_{K}$. If $K$ is an Archimedean local field, we write $\operatorname{rec}_{K}$ for the local Langlands correspondence of [Lan89]. We will write rec for $\operatorname{rec}_{K}$ when the choice of $K$ is clear.

\section{An AUtOMORPhy LIFTING THEOREM}

3.1. The group $\mathcal{G}_{n}$. Let $n$ be a positive integer, and let $\mathcal{G}_{n}$ be the group scheme over $\mathbb{Z}$ which is the semidirect product of $\mathrm{GL}_{n} \times \mathrm{GL}_{1}$ by the group $\{1, j\}$, which acts on $\mathrm{GL}_{n} \times \mathrm{GL}_{1}$ by

$$
j(g, \mu) j^{-1}=\left(\mu^{t} g^{-1}, \mu\right) .
$$

There is a homomorphism $\nu: \mathcal{G}_{n} \rightarrow \mathrm{GL}_{1}$ sending $(g, \mu)$ to $\mu$ and $j$ to -1 . Write $\mathfrak{g}_{n}^{0}$ for the trace zero subspace of the Lie algebra of $\mathrm{GL}_{n}$, regarded as a Lie subalgebra of the Lie algebra of $\mathcal{G}_{n}$.

Suppose that $F$ is an imaginary CM field with totally real subfield $F^{+}$. If $R$ is a ring and $r: G_{F^{+}} \rightarrow \mathcal{G}_{n}(R)$ is a homomorphism with $r^{-1}\left(\mathrm{GL}_{n}(R) \times \mathrm{GL}_{1}(R)\right)=G_{F}$, we will make a slight abuse of notation and write $\left.r\right|_{G_{F}}$ (respectively $\left.r\right|_{G_{F_{w}}}$ for $w$ a place of $F$ ) to mean $\left.r\right|_{G_{F}}$ (respectively $\left.r\right|_{G_{F_{w}}}$ ) composed with the projection $\mathrm{GL}_{n}(R) \times \mathrm{GL}_{1}(R) \rightarrow \mathrm{GL}_{n}(R)$. 
3.2. l-adic automorphic forms on unitary groups. Let $F^{+}$denote a totally real number field and $n$ a positive integer. Let $F / F^{+}$be a totally imaginary quadratic extension of $F^{+}$and let $c$ denote the non-trivial element of $\operatorname{Gal}\left(F / F^{+}\right)$. Suppose that the extension $F / F^{+}$is unramified at all finite places. Assume that $n\left[F^{+}: \mathbb{Q}\right]$ is divisible by 4 . Under this assumption, we can find a reductive algebraic group $G$ over $F^{+}$with the following properties:

- $G$ is an outer form of $\mathrm{GL}_{n}$ with $G_{/ F} \cong \mathrm{GL}_{n / F}$;

- for every finite place $v$ of $F^{+}, G$ is quasi-split at $v$;

- for every infinite place $v$ of $F^{+}, G\left(F_{v}^{+}\right) \cong U_{n}(\mathbb{R})$.

We can and do fix a model for $G$ over the ring of integers $\mathcal{O}_{F^{+}}$of $F^{+}$as in section 2.1 of Ger09. For each place $v$ of $F^{+}$which splits as $w w^{c}$ in $F$ there is a natural isomorphism

$$
\iota_{w}: G\left(F_{v}^{+}\right) \stackrel{\sim}{\longrightarrow} G L_{n}\left(F_{w}\right)
$$

which restricts to an isomorphism between $G\left(\mathcal{O}_{F_{v}^{+}}\right)$and $G L_{n}\left(\mathcal{O}_{F_{w}}\right)$. If $v$ is a place of $F^{+}$split over $F$ and $w$ is a place of $F$ dividing $v$, then we let

- $\operatorname{Iw}(w)$ denote the subgroup of $G L_{n}\left(\mathcal{O}_{F_{w}}\right)$ consisting of matrices which reduce to an upper triangular matrix modulo $w$;

- $U_{0}(w)$ denote the subgroup of $G L_{n}\left(\mathcal{O}_{F_{w}}\right)$ consisting of matrices whose last row is congruent to $(0, \ldots, 0, *)$ modulo $w$;

- $U_{1}(w)$ denote the subgroup of $U_{0}(w)$ consisting of matrices whose last row is congruent to $(0, \ldots, 0,1)$ modulo $w$.

Let $l>n$ be a prime number with the property that every place of $F^{+}$dividing $l$ splits in $F$. Let $S_{l}$ denote the set of places of $F^{+}$dividing $l$. Let $K$ be an algebraic extension of $\mathbb{Q}_{l}$ in $\overline{\mathbb{Q}}_{l}$ such that every embedding $F \hookrightarrow \overline{\mathbb{Q}}_{l}$ has its image contained in $K$. Let $\mathcal{O}$ denote the ring of integers in $K$ and $k$ the residue field. Let $S_{l}$ denote the set of places of $F^{+}$dividing $l$ and for each $v \in S_{l}$, let $\widetilde{v}$ be a place of $F$ over $v$.

Let $W$ be an $\mathcal{O}$-module with an action of $G\left(\mathcal{O}_{F^{+}, l}\right)$. Let $V \subset G\left(\mathbb{A}_{F^{+}}^{\infty}\right)$ be a compact open subgroup with $v_{l} \in G\left(\mathcal{O}_{F^{+}, l}\right)$ for all $v \in V$, where $v_{l}$ denotes the projection of $v$ to $G\left(F_{l}^{+}\right)$. We let $S(V, W)$ denote the space of $l$-adic automorphic forms on $G$ of weight $W$ and level $V$, that is, the space of functions

$$
f: G\left(F^{+}\right) \backslash G\left(\mathbb{A}_{F^{+}}^{\infty}\right) \rightarrow W
$$

with $f(g v)=v_{l}^{-1} f(g)$ for all $v \in V$.

Let $\widetilde{I}_{l}$ denote the set of embeddings $F \hookrightarrow K$ giving rise to one of the places $\widetilde{v}$. Let $\left(\mathbb{Z}_{+}^{n}\right)^{\widetilde{I}_{l}}$ denote the set of $\lambda \in\left(\mathbb{Z}^{n}\right)^{\widetilde{I}_{l}}$ with $\lambda_{\tau, 1} \geq \lambda_{\tau, 2} \geq \cdots \geq \lambda_{\tau, n}$ for all embeddings $\tau \in \widetilde{I}_{l}$. To each $\lambda \in\left(\mathbb{Z}_{+}^{n}\right)^{\widetilde{I}_{l}}$ we associate a finite free $\mathcal{O}$-module $M_{\lambda}$ with a continuous action of $G\left(\mathcal{O}_{F^{+}, l}\right)$ as in Definition 2.2.3 of Ger09. The representation $M_{\lambda}$ is the tensor product over $\tau \in \widetilde{I}_{l}$ of the irreducible algebraic representations of $\mathrm{GL}_{n}$ of highest weights given by the $\lambda_{\tau}$. We write $S_{\lambda}(V, \mathcal{O})$ instead of $S\left(V, M_{\lambda}\right)$ and similarly for any $\mathcal{O}$-module $A$, we write $S_{\lambda}(V, A)$ for $S\left(V, M_{\lambda} \otimes_{\mathcal{O}} A\right)$.

Assume from now on that $K$ is a finite extension of $\mathbb{Q}_{l}$. Let $\mathfrak{l}$ denote the product of all places in $S_{l}$. Let $R$ and $S_{a}$ denote finite sets of finite places of $F^{+}$disjoint from each other and from $S_{l}$ and consisting only of places which split in $F$. Assume that each $v \in S_{a}$ is unramified over a rational prime $p$ with $\left[F\left(\zeta_{p}\right): F\right]>n$. Let $T=S_{l} \sqcup R \sqcup S_{a}$. For each $v \in T$ fix a place $\widetilde{v}$ of $F$ dividing $v$, extending the choice of $\widetilde{v}$ for $v \in S_{l}$. Let $U=\prod_{v} U_{v}$ be a compact open subgroup of $G\left(\mathbb{A}_{F^{+}}^{\infty}\right)$ such that

$$
\text { - } U_{v}=G\left(\mathcal{O}_{F_{v}^{+}}\right) \text {if } v \notin R \cup S_{a} \text { splits in } F \text {; }
$$


- $U_{v}=\iota_{\widetilde{v}}^{-1} \operatorname{ker}\left(G L_{n}\left(\mathcal{O}_{F_{\widetilde{v}}}\right) \rightarrow G L_{n}(k(\widetilde{v}))\right)$ if $v \in S_{a}$;

- $U_{v}$ is a hyperspecial maximal compact subgroup of $G\left(F_{v}^{+}\right)$if $v$ is inert in $F$.

(At this stage we impose no restrictions on $U_{v}$ for places $v \in R$.) We note that if $S_{a}$ is non-empty, then $U$ is sufficiently small (which means that its projection to $G\left(F_{v}^{+}\right)$for some place $v \in F^{+}$contains no finite order elements other than the identity).

For any $\mathcal{O}$-algebra $A$, the space $S_{\lambda}(U, A)$ is acted upon by the Hecke operators

$$
T_{w}^{(j)}:=\iota_{w}^{-1}\left(\left[G L_{n}\left(\mathcal{O}_{F_{w}}\right)\left(\begin{array}{cc}
\varpi_{w} 1_{j} & 0 \\
0 & 1_{n-j}
\end{array}\right) G L_{n}\left(\mathcal{O}_{F_{w}}\right)\right]\right)
$$

for $w$ a place of $F$, split over $F^{+}$and not lying over $T, j=1, \ldots, n$ and $\varpi_{w}$ a uniformizer in $\mathcal{O}_{F_{w}}$. We let $\mathbb{T}_{\lambda}^{T}(U, A)$ be the $A$-subalgebra of $\operatorname{End}_{A}\left(S_{\lambda}(U, A)\right)$ generated by these operators and the operators $\left(T_{w}^{(n)}\right)^{-1}$. tion

To any maximal ideal $\mathfrak{m}$ of $\mathbb{T}_{\lambda}^{T}(U, \mathcal{O})$ one can associate a continuous representa-

characterised by the following properties:

$$
\bar{r}_{\mathfrak{m}}: G_{F} \rightarrow \mathrm{GL}_{n}\left(\mathbb{T}_{\lambda}^{T}(U, \mathcal{O}) / \mathfrak{m}\right)
$$

(1) $\bar{r}_{\mathfrak{m}}^{c} \cong \bar{r}_{\mathfrak{m}}^{\vee} \bar{\epsilon}^{1-n}$.

(2) $\bar{r}_{\mathfrak{m}}$ is unramified outside $T$. If $v \notin T$ is a place of $F^{+}$which splits as $w w^{c}$ in $F$ and Frob $_{w}$ is the geometric Frobenius element of $G_{F_{w}} / I_{F_{w}}$, then $\bar{r}_{\mathfrak{m}}\left(\right.$ Frob $\left._{w}\right)$ has characteristic polynomial

$$
X^{n}+\cdots+(-1)^{j}(\mathbf{N} w)^{j(j-1) / 2} T_{w}^{(j)} X^{n-j}+\cdots+(-1)^{n}(\mathbf{N} w)^{n(n-1) / 2} T_{w}^{(n)} .
$$

The maximal ideal $\mathfrak{m}$ is said to be non-Eisenstein if $\bar{r}_{\mathfrak{m}}$ is absolutely irreducible. In this case, $\bar{r}_{\mathfrak{m}}$ can be extended to a homomorphism $\bar{r}_{\mathfrak{m}}: G_{F^{+}} \rightarrow \mathcal{G}_{n}\left(\mathbb{T}_{\lambda}^{T}(U, \mathcal{O}) / \mathfrak{m}\right)$ (in the sense that $\left.\left.\bar{r}_{\mathfrak{m}}\right|_{G_{F}}=\left(\bar{r}_{\mathfrak{m}}, \bar{\epsilon}^{1-n}\right)\right)$ with $\bar{r}_{\mathfrak{m}}^{-1}\left(\left(\mathrm{GL}_{n} \times \mathrm{GL}_{1}\right)\left(\mathbb{T}_{\lambda}^{T}(U, \mathcal{O}) / \mathfrak{m}\right)\right)=G_{F}$. Also, any such extension has a continuous lifting

$$
r_{\mathfrak{m}}: G_{F^{+}} \rightarrow \mathcal{G}_{n}\left(\mathbb{T}_{\lambda}^{T}(U, \mathcal{O})_{\mathfrak{m}}\right)
$$

with the following properties:

(0) $r_{\mathfrak{m}}^{-1}\left(\left(\mathrm{GL}_{n} \times \mathrm{GL}_{1}\right)\left(\mathbb{T}_{\lambda}^{T}(U, \mathcal{O})_{\mathfrak{m}}\right)\right)=G_{F}$.

(1) $\nu \circ r_{\mathfrak{m}}=\epsilon^{1-n} \delta_{F / F^{+}}^{\mu_{\mathfrak{m}}}$, where $\delta_{F / F^{+}}$is the non-trivial character of $\operatorname{Gal}\left(F / F^{+}\right)$ and $\mu_{\mathfrak{m}} \in \mathbb{Z} / 2 \mathbb{Z}$.

(2) $r_{\mathfrak{m}}$ is unramified outside $T$. If $v \notin T$ is a place of $F^{+}$which splits as $w w^{c}$ in $F$ and Frob $_{w}$ is the geometric Frobenius element of $G_{F_{w}} / I_{F_{w}}$, then $r_{\mathfrak{m}}\left(\operatorname{Frob}_{w}\right)$ has characteristic polynomial

$$
X^{n}+\cdots+(-1)^{j}(\mathbf{N} w)^{j(j-1) / 2} T_{w}^{(j)} X^{n-j}+\cdots+(-1)^{n}(\mathbf{N} w)^{n(n-1) / 2} T_{w}^{(n)} .
$$

(3) If $v \in S_{l}$, and $\zeta: \mathbb{T}_{\lambda}^{T}(U, \mathcal{O}) \rightarrow \overline{\mathbb{Q}}_{l}$ is a homomorphism of $\mathcal{O}$-algebras, then $\left.\zeta \circ r_{\mathfrak{m}}\right|_{G_{\tilde{v}}}$ is crystalline of $l$-adic Hodge type $\mathbf{v}_{\lambda_{\tilde{v}}}$ (in the sense of Definition 3.3 .4 below).

3.3. Local deformation rings. Let $l$ be a prime number and $K$ be a finite extension of $\mathbb{Q}_{l}$ with residue field $k$ and ring of integers $\mathcal{O}$, and write $\mathfrak{m}_{\mathcal{O}}$ for the maximal ideal of $\mathcal{O}$. Let $\mathcal{C}_{\mathcal{O}}$ be the category of complete local Noetherian $\mathcal{O}$-algebras with residue field isomorphic to $k$ via the structural homomorphism. As in section 3 of BLGHT09, we consider an object $R$ of $\mathcal{C}_{\mathcal{O}}$ to be geometrically integral if for all finite extensions $K^{\prime} / K$, the algebra $R \otimes_{\mathcal{O}} \mathcal{O}_{K^{\prime}}$ is an integral domain. 
Let $M$ be a finite extension of $\mathbb{Q}_{p}$ for some prime $p$ possibly equal to $l$ and let $\bar{\rho}: G_{M} \rightarrow \mathrm{GL}_{n}(k)$ be a continuous homomorphism. Then the functor from $\mathcal{C}_{\mathcal{O}}$ to Sets which takes $A \in \mathcal{C}_{\mathcal{O}}$ to the set of continuous liftings $\rho: G_{M} \rightarrow \mathrm{GL}_{n}(A)$ of $\bar{\rho}$ is represented by a complete local Noetherian $\mathcal{O}$-algebra $R_{\bar{\rho}}^{\square}$. We call this ring the universal $\mathcal{O}$-lifting ring of $\bar{\rho}$. We write $\rho^{\square}: G_{M} \rightarrow \mathrm{GL}_{n}\left(R_{\bar{\rho}}^{\square}\right)$ for the universal lifting.

The following definitions will prove to be useful later.

Definition 3.3.1. Suppose $\rho: G_{M} \rightarrow \mathrm{GL}_{2}(\mathcal{O})$ is a representation. We can think of this as putting a $G_{M}$-action on the vector space $K^{2}(=V$, say), in a way that stabilises the lattice generated by the standard basis $\left\{e_{0}, e_{1}\right\}$, where $e_{0}=\langle 1,0\rangle$, $e_{1}=\langle 0,1\rangle$. Considering $\operatorname{Sym}^{n-1} \rho$ as a quotient of $V^{\otimes(n-1)}$, we have an ordered basis $\left\{g_{0}, \ldots, g_{n-1}\right\}$ of $\operatorname{Sym}^{n-1} V$, where $g_{i}$ is the image of $e_{0}^{\otimes(n-1-i)} \otimes e_{1}^{\otimes i}$. We call this the $\mathcal{O}$-basis of $\mathrm{Sym}^{n-1} V$ inherited from our original basis in $\rho$.

Definition 3.3.2. Suppose $\rho: G_{M} \rightarrow \mathrm{GL}_{n}(\mathcal{O}), \rho^{\prime}: G_{M} \rightarrow \mathrm{GL}_{n^{\prime}}(\mathcal{O})$ are representations, which we think of as putting $G_{M}$-actions on the vector spaces $V_{\rho}=K^{n}$, $V_{\rho}^{\prime}=K^{n^{\prime}}$ in a way that stabilises the lattices generated by the standard bases of each. In this situation we have an ordered $\mathcal{O}$-basis on $V_{\rho} \otimes_{\mathcal{O}} V_{\rho}^{\prime}$ given by the vectors $e_{j} \otimes f_{k}$, ordered lexicographically, where the $e_{j}$ are the standard $\mathcal{O}$-basis in $V_{\rho}$ and the $f_{k}$ are the standard basis in $V_{\rho}^{\prime}$. We call this the $\mathcal{O}$-basis of $\rho \otimes_{\mathcal{O}} \rho^{\prime}$ inherited from our original bases.

3.3.1. Local deformations $(p=l$ case). Suppose that $p=l$. In this section we will define an equivalence relation on crystalline lifts of $\bar{\rho}$. For this, we need to consider certain quotients of $R_{\bar{\rho}}^{\square}$. Assume that $K$ contains the image of every embedding $M \hookrightarrow \bar{K}$.

Definition 3.3.3. Let $\left(\mathbb{Z}_{+}^{n}\right)^{\operatorname{Hom}(M, K)}$ denote the subset of $\left(\mathbb{Z}^{n}\right)^{\operatorname{Hom}(M, K)}$ consisting of elements $\lambda$ which satisfy

$$
\lambda_{\tau, 1} \geq \lambda_{\tau, 2} \geq \cdots \geq \lambda_{\tau, n}
$$

for every embedding $\tau$.

Let $\lambda$ be an element of $\left(\mathbb{Z}_{+}^{n}\right)^{\operatorname{Hom}(M, K)}$. We associate to $\lambda$ an $l$-adic Hodge type $\mathbf{v}_{\lambda}$ in the sense of Section 2.6 of [Kis08] as follows. Let $D_{K}$ denote the vector space $K^{n}$. Let $D_{K, M}=D_{K} \otimes_{\mathbb{Q}_{l}} M$. For each embedding $\tau: M \hookrightarrow K$, we let $D_{K, \tau}=$ $D_{K, M} \otimes_{K \otimes M, 1 \otimes \tau} K$ so that $D_{K, M}=\bigoplus_{\tau} D_{K, \tau}$. For each $\tau$ choose a decreasing filtration $\mathrm{Fil}^{i} D_{K, \tau}$ of $D_{K, \tau}$ so that $\operatorname{dim}_{K} \operatorname{gr}^{i} D_{K, \tau}=0$ unless $i=(j-1)+\lambda_{\tau, n-j+1}$ for some $j=1, \ldots, n$ in which case $\operatorname{dim}_{K} \operatorname{gr}^{i} D_{K, \tau}=1$. We define a decreasing filtration of $D_{K, M}$ by $K \otimes_{\mathbb{Q}_{l}} M$-submodules by setting

$$
\mathrm{Fil}^{i} D_{K, M}=\bigoplus_{\tau} \mathrm{Fil}^{i} D_{K, \tau}
$$

Let $\mathbf{v}_{\lambda}=\left\{D_{K}, \mathrm{Fil}^{i} D_{K, M}\right\}$.

We now recall some results of Kisin. Let $\lambda$ be an element of $\left(\mathbb{Z}_{+}^{n}\right)^{\operatorname{Hom}(M, K)}$ and let $\mathbf{v}_{\lambda}$ be the associated $l$-adic Hodge type.

Definition 3.3.4. If $B$ is a finite $K$-algebra and $V_{B}$ is a free $B$-module of rank $n$ with a continuous action of $G_{M}$ that makes $V_{B}$ into a de Rham representation, 
then we say that $V_{B}$ is of l-adic Hodge type $\mathbf{v}_{\lambda}$ if for each $i$ there is an isomorphism of $B \otimes \mathbb{Q}_{l} M$-modules

$$
\operatorname{gr}^{i}\left(V_{B} \otimes_{\mathbb{Q}_{l}} B_{d R}\right)^{G_{M}} \stackrel{\sim}{\rightarrow}\left(\mathrm{gr}^{i} D_{K, M}\right) \otimes_{K} B .
$$

Corollary 2.7.7 of [Kis08] implies that there is a unique $l$-torsion free quotient $R_{\bar{\rho}}^{\mathbf{v}_{\lambda}, c r}$ of $R_{\bar{\rho}}^{\square}$ with the property that for any finite $K$-algebra $B$, a homomorphism of $\mathcal{O}$-algebras $\zeta: R_{\bar{\rho}}^{\square} \rightarrow B$ factors through $R_{\bar{\rho}}^{\mathbf{v}_{\lambda}, c r}$ if and only if $\zeta \circ \rho^{\square}$ is crystalline of $l$-adic Hodge type $\mathbf{v}_{\lambda}$. Moreover, Theorem 3.3.8 of Kis08 implies that $\operatorname{Spec} R_{\bar{\rho}}^{\mathbf{v}_{\lambda}, c r}[1 / l]$ is formally smooth over $K$ and equidimensional of dimension $n^{2}+\frac{1}{2} n(n-1)\left[M: \mathbb{Q}_{l}\right]$.

By Lemma 3.3.3 of Ger09] there is a quotient $R_{\bar{\rho}}^{\triangle_{\lambda}, c r}$ of $R_{\bar{\rho}}^{\mathbf{v}_{\lambda}, c r}$ corresponding to a union of irreducible components such that for any finite extension $E$ of $K$, a homomorphism of $\mathcal{O}$-algebras $\zeta: R_{\bar{\rho}}^{\mathbf{v}_{\lambda}, c r} \rightarrow E$ factors through $R_{\bar{\rho}}^{\triangle_{\lambda}, c r}$ if and only if $\zeta \circ \rho^{\square}$ is crystalline and ordinary of weight $\lambda$.

We now introduce an equivalence relation on continuous representations $G_{M} \rightarrow$ $\mathrm{GL}_{n}(\mathcal{O})$ lifting $\bar{\rho}$.

Definition 3.3.5. Suppose that $\rho_{1}, \rho_{2}: G_{M} \rightarrow \operatorname{GL}_{n}(\mathcal{O})$ are two continuous lifts of $\bar{\rho}$. Then we say that $\rho_{1} \sim \rho_{2}$ if the following hold.

(1) There is a $\lambda \in\left(\mathbb{Z}_{+}^{n}\right)^{\operatorname{Hom}(M, K)}$ such that $\rho_{1}$ and $\rho_{2}$ both correspond to points of $R_{\bar{\rho}}^{\mathbf{v}_{\lambda}, c r}$ (that is, $\rho_{1} \otimes_{\mathcal{O}} K$ and $\rho_{2} \otimes_{\mathcal{O}} K$ are both crystalline of $l$-adic Hodge type $\mathbf{v}_{\lambda}$ ).

(2) For every minimal prime ideal $\wp$ of $R_{\bar{\rho}}^{\mathbf{v}_{\lambda}, c r}$, the quotient $R_{\bar{\rho}}^{\mathbf{v}_{\lambda}, c r} / \wp$ is geometrically integral.

(3) $\rho_{1}$ and $\rho_{2}$ give rise to closed points on a common irreducible component of $\operatorname{Spec} R_{\bar{\rho}}^{\mathbf{v}_{\lambda}, c r}[1 / l]$.

In (3) above, note that the irreducible component is uniquely determined by either of $\rho_{1}, \rho_{2}$ because $\operatorname{Spec} R_{\bar{\rho}}^{\mathbf{v}_{\lambda}, c r}[1 / l]$ is formally smooth. Note also that we can always ensure that (2) holds by replacing $\mathcal{O}$ with the ring of integers in a finite extension of $K$.

Suppose that $\rho_{1} \sim \rho_{2}$ as above and let $M^{\prime} / M$ be a finite extension. Assume that $K$ contains the image of every embedding $M^{\prime} \hookrightarrow \bar{K}$. Then we claim that $\left.\left.\rho_{1}\right|_{G_{M^{\prime}}} \sim \rho_{2}\right|_{G_{M^{\prime}}}$. Indeed, let $\lambda$ be such that $\rho_{1}$ and $\rho_{2}$ have $l$-adic Hodge type $\mathbf{v}_{\lambda}$. Define $\lambda^{\prime} \in\left(\mathbb{Z}_{+}^{n}\right)^{\operatorname{Hom}\left(M^{\prime}, K\right)}$ by $\lambda_{\tau}^{\prime}=\lambda_{\left.\tau\right|_{M}}$ for all $\tau: M^{\prime} \hookrightarrow K$. Then restriction to $G_{M^{\prime}}$ gives rise to an $\mathcal{O}$-algebra homomorphism $R_{\left.\bar{\rho}\right|_{G_{M^{\prime}}} ^{\square}}^{\square} R_{\bar{\rho}}^{\mathbf{v}_{\lambda}, c r}$ which factors

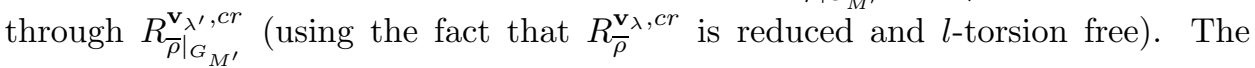
result now follows from the formal smoothness of $\operatorname{Spec} R_{\left.\bar{\rho}\right|_{G_{M}}}^{\mathbf{v}_{\lambda^{\prime}}, c r}[1 / l]$, which implies that the image of any irreducible component of $\operatorname{Spec} R_{\bar{\rho}}^{\mathbf{v}_{\lambda}, c r}[1 / l]$ is contained in a unique irreducible component of $\operatorname{Spec} R_{\left.\bar{\rho}\right|_{G_{M^{\prime}}}}^{\mathbf{v}_{\lambda^{\prime}}, c r}[1 / l]$.

In a similar vein, it follows that if $n=2$ and $\rho_{1} \sim \rho_{2}$, then $\operatorname{Sym}^{k-1} \rho_{1} \sim$ $\operatorname{Sym}^{k-1} \rho_{2}$ for all $k \geq 1$, where we take the $\mathcal{O}$-basis on the $\operatorname{Sym}^{k-1} \rho_{i}$ inherited from the bases we have on the $\rho_{i}$, in the sense of Definition 3.3.1. [The same is true if $n>2$, with an appropriate modification of Definition 3.3.1]

We will make one final variation on this theme. Suppose $\rho^{\prime}: G_{M} \rightarrow \mathrm{GL}_{m}(\mathcal{O})$ is crystalline of $l$-adic Hodge type $\mathbf{v}_{\lambda^{\prime}}$ for some $m$ and some $\lambda^{\prime} \in\left(\mathbb{Z}_{+}^{m}\right)^{\operatorname{Hom}(M, K)}$, and $\rho_{1} \sim \rho_{2}$ are as above. (Note that $n$ need no longer be 2.) Then $\rho_{1} \otimes_{\mathcal{O}} \rho^{\prime} \sim \rho_{2} \otimes_{\mathcal{O}} \rho^{\prime}$, 
where we take as $\mathcal{O}$-basis on the $\rho_{i} \otimes_{\mathcal{O}} \rho^{\prime}$ the inherited bases in the sense of Definition 3.3 .2 .

Lemma 3.3.6. Let $\bar{\rho}: G_{M} \rightarrow \mathrm{GL}_{n}(k)$ be a continuous homomorphism. Suppose $\rho_{1}, \rho_{2}: G_{M} \rightarrow \mathrm{GL}_{n}(\mathcal{O})$ are two lifts of $\bar{\rho}$ with $\rho_{1} \sim \rho_{2}$. If $\mathcal{O}^{\prime}$ denotes the ring of integers in a finite extension of $K$ with residue field $k^{\prime}$, then $\rho_{1} \sim \rho_{2}$, regarded as lifts of $\bar{\rho} \otimes_{k} k^{\prime}$ to $\mathrm{GL}_{n}\left(\mathcal{O}^{\prime}\right)$.

Proof. Let $\lambda \in\left(\mathbb{Z}_{+}^{n}\right)^{\operatorname{Hom}(M, K)}$ be such that $\rho_{1}$ and $\rho_{2}$ have $l$-adic Hodge type $\mathbf{v}_{\lambda}$. Let $R=R_{\bar{\rho}}^{\mathbf{v}_{\lambda}, c r}$ and $R^{\prime}=R \otimes_{\mathcal{O}} \mathcal{O}^{\prime}$. We need to show that $\rho_{1}$ and $\rho_{2}$ give rise to closed points of Spec $R^{\prime}[1 / l]$ lying on a common component. Note that if $\mathcal{C}^{\prime}$ is an irreducible component of $\operatorname{Spec} R^{\prime}[1 / l]$, then the image of $\mathcal{C}^{\prime}$ in $\operatorname{Spec} R[1 / l]$ is an irreducible component. Indeed, the image of $\mathcal{C}^{\prime}$ in Spec $R[1 / l]$ is irreducible and closed (as $R \rightarrow R^{\prime}$ is finite). If $x^{\prime}$ is a closed point of $\operatorname{Spec} R^{\prime}[1 / l]$ lying in $\mathcal{C}^{\prime}$ with image $x$ in Spec $R[1 / l]$, then the completed local rings of Spec $R^{\prime}[1 / l]$ and Spec $R[1 / l]$ at $x^{\prime}$ and $x$ respectively are isomorphic. We deduce that the image of $\mathcal{C}^{\prime}$ has the same dimension as $\mathcal{C}^{\prime}$ and hence is an irreducible component.

Now, let $x_{1}$ and $x_{2}$ denote the closed points of $\operatorname{Spec} R[1 / l]$ corresponding to $\rho_{1}$ and $\rho_{2}$ and let $\mathcal{C}$ denote the irreducible component of Spec $R[1 / l]$ containing $x_{1}$ and $x_{2}$. Then we claim that the preimage of $\mathcal{C}$ in $\operatorname{Spec} R^{\prime}[1 / l]$ is irreducible. Indeed, suppose there are two distinct irreducible components $\mathcal{C}^{\prime}$ and $\mathcal{C}^{\prime \prime}$ of $\operatorname{Spec} R^{\prime}[1 / l]$ mapping to $\mathcal{C}$. Then there are points $x_{1}^{\prime}$ and $x_{1}^{\prime \prime}$ of $\mathcal{C}^{\prime}$ and $\mathcal{C}^{\prime \prime}$ respectively mapping to $x_{1}$. However, the preimage of $x_{1}$ in Spec $R^{\prime}[1 / l]$ consists of a single point. (Let $\mathfrak{m}$ denote the maximal ideal of $R[1 / l]$ corresponding to $x_{1}$. Then the fibre over $x_{1}$ is given by the spectrum of $(R[1 / l] / \mathfrak{m}) \otimes_{\mathcal{O}} \mathcal{O}^{\prime} \cong K \otimes_{\mathcal{O}} \mathcal{O}^{\prime} \cong K^{\prime}$.) Thus $x_{1}^{\prime}=$ $x_{1}^{\prime \prime}$ lies in the intersection of $\mathcal{C}^{\prime}$ and $\mathcal{C}^{\prime \prime}$, contradicting the formal smoothness of $\operatorname{Spec} R^{\prime}[1 / l]$.

3.3.2. Local deformations $(p \neq l$ case). Suppose now that $p \neq l$. By Theorem 2.1.6 of [Gee10], Spec $R_{\bar{\rho}}^{\square}[1 / l]$ is equidimensional of dimension $n^{2}$.

Definition 3.3.7. Let $\rho_{1}, \rho_{2}: G_{M} \rightarrow \mathrm{GL}_{n}(\mathcal{O})$ be two lifts of $\bar{\rho}$. We say that $\rho_{1} \rightsquigarrow \mathcal{O} \rho_{2}$ if the following hold.

(1) For each minimal prime ideal $\wp$ of $R^{\square}$, the quotient $R^{\square} / \wp$ is geometrically irreducible.

(2) $\rho_{1}$ corresponds to a closed point of $\operatorname{Spec} R_{\bar{\rho}}^{\square}[1 / l]$ which is contained in a unique irreducible component and this irreducible component also contains the closed point corresponding to $\rho_{2}$.

We remark that we can always replace $\mathcal{O}$ by the ring of integers in a finite extension of $K$ so that condition (1) above holds. Also, condition (1) ensures that if $\rho_{1} \rightsquigarrow \mathcal{O} \rho_{2}$ and if $\mathcal{O}^{\prime}$ is the ring of integers in a finite extension of $K$, then $\rho_{1} \rightsquigarrow \mathcal{O}^{\prime} \rho_{2}$.

\subsection{Properties of $\rightsquigarrow \mathcal{O}$ and $\sim$.}

Lemma 3.4.1. $\sim$ is an equivalence relation.

Proof. This follows immediately from the definitions.

Lemma 3.4.2. Let $M$ be a finite extension of $\mathbb{Q}_{p}$ for some prime p. Let $\bar{\rho}: G_{M} \rightarrow$ $\mathrm{GL}_{n}(k)$ be a continuous homomorphism. If $p \neq l$, let $R=R_{\bar{\rho}}^{\square}$. If $p=l$, assume that $K$ contains the image of each embedding $M \hookrightarrow \overline{\mathbb{Q}}_{l}$ and let $R=R_{\bar{\rho}}^{\mathbf{v}_{\lambda}, \text { cr }}$ for 
some $\lambda \in\left(\mathbb{Z}_{+}^{n}\right)^{\operatorname{Hom}(M, K)}$. Let $\mathcal{O}^{\prime}$ denote the ring of integers in a finite extension of $K$. Let $\rho$ and $\rho^{\prime}$ be two lifts of $\bar{\rho}$ to $\mathcal{O}^{\prime}$ giving rise to closed points of Spec $R[1 / l]$. Suppose that after conjugation by an element of $\operatorname{ker}\left(G L_{n}\left(\mathcal{O}^{\prime}\right) \rightarrow \operatorname{GL}_{n}\left(\mathcal{O}^{\prime} / \mathfrak{m}_{\mathcal{O}^{\prime}}\right)\right)$ they differ by an unramified twist. Then an irreducible component of $\operatorname{Spec} R[1 / l]$ contains $\rho$ if and only if it contains $\rho^{\prime}$.

Proof. The universal unramified $\mathcal{O}$-lifting ring of the trivial character $G_{M} \rightarrow k^{\times}$is given by $\mathcal{O}[[Y]]$ where the universal lift $\chi^{\square}$ sends Frob ${ }_{M}$ to $1+Y$. Let $R[[Y, \underline{X}]]=$ $R[[Y]]\left[\left[X_{i j}: 1 \leq i, j \leq n\right]\right]$. Let $\rho^{\square}$ denote the universal lift of $\bar{\rho}$ to $R$. Consider the lift $\left(1_{n}+\left(X_{i j}\right)\right) \rho^{\square}\left(1_{n}+\left(X_{i j}\right)\right)^{-1} \otimes \chi^{\square}$ of $\bar{\rho}$ to $R[[Y, \underline{X}]]$. This lift gives rise to a homomorphism $R_{\bar{\rho}}^{\square} \rightarrow R[[Y, \underline{X}]]$ which factors through $R$. Let $\alpha$ denote the resulting $\mathcal{O}$-algebra homomorphism $R \rightarrow R[[Y, \underline{X}]]$. Let $\iota: R \rightarrow R[[Y, \underline{X}]]$ be the standard $R$-algebra structure on $R[[Y, \underline{X}]]$.

The minimal prime ideals of $R[[Y, \underline{X}]]$ and $R$ are in natural bijection (if $\wp$ is a minimal prime of $R$, then $\iota(\wp)$ generates a minimal prime of $R[[Y, \underline{X}]])$. Let $\wp$ be a minimal prime of $R$. We claim that the kernel of the map $\beta: R \rightarrow$ $R[[Y, \underline{X}]] / \iota(\wp)=(R / \wp)[[Y, \underline{X}]]$ induced by $\alpha$ is $\wp$. Indeed, the $R$-algebra homomorphism (with $R[[Y, \underline{X}]]$ considered as an $R$-algebra via $\iota) \gamma: R[[Y, \underline{X}]] \rightarrow R$ which sends $Y$ and each $X_{i j}$ to 0 is a section to the map $\beta$. The composition $R \stackrel{\beta}{\rightarrow}(R / \wp)[[Y, \underline{X}]] \stackrel{\gamma}{\rightarrow} R / \wp$ is thus the natural reduction map. In particular its kernel is $\wp$. Since $\operatorname{ker}(\beta) \subset \operatorname{ker}(\gamma \circ \beta)=\wp$ and $\wp$ is minimal, we deduce that $\operatorname{ker}(\beta)=\wp$. The lemma follows.

Lemma 3.4.3. Let $M$ be a finite extension of $\mathbb{Q}_{l}$. Let $\bar{\rho}: G_{M} \rightarrow \operatorname{GL}_{n}(k)$ be the trivial representation, and let $\rho$ and $\rho^{\prime}: G_{M} \rightarrow \mathrm{GL}_{n}(\mathcal{O})$ be two crystalline lifts of $\bar{\rho}$ of l-adic Hodge type $\mathbf{v}_{\lambda}$ which are $\mathrm{GL}_{n}(\mathcal{O})$-conjugate. Then $\rho \sim \rho^{\prime}$.

Proof. Take $g \in \mathrm{GL}_{n}(\mathcal{O})$ with $\rho^{\prime}=g \rho g^{-1}$. Let $A=\mathcal{O}\left\langle X_{i j}, Y\right\rangle /\left(Y \operatorname{det}\left(X_{i j}\right)-1\right)$, where $\mathcal{O}\left\langle X_{i j}, Y\right\rangle$ is the $\mathfrak{m}_{\mathcal{O}}$-adic completion of $\mathcal{O}\left[X_{i j}, Y\right]$. Let $\rho_{A}: G_{M} \rightarrow \mathrm{GL}_{n}(A)$ be given by $X \rho X^{-1}$, where $X$ is the matrix $\left(X_{i j}\right)$. By Lemma 3.3.1 of Ger09], there is a continuous homomorphism $R_{\bar{\rho}}^{\square} \rightarrow A$ such that $\rho_{A}$ is the push-forward of the universal lifting $\rho^{\square}: G_{M} \rightarrow \mathrm{GL}_{n}\left(R_{\bar{\rho}}^{\square}\right)$. Now, for any $\overline{\mathbb{Q}}_{l}$-point of $A$, the corresponding specialisation of $\rho_{A}$ is a $\overline{\mathbb{Q}}_{l}$-conjugate of $\rho$, and is thus crystalline of $l$-adic Hodge type $\mathbf{v}_{\lambda}$, so corresponds to a $\overline{\mathbb{Q}}_{l}$-point of $R_{\bar{\rho}}^{\mathbf{v}_{\lambda}, c r}$. Since the $\overline{\mathbb{Q}}_{l}$-points of $A$ are dense in $\operatorname{Spec} A$, we conclude that the homomorphism $R_{\bar{\rho}}^{\square} \rightarrow A$ factors through $R_{\bar{\rho}}^{\mathbf{v}_{\lambda}, c r}$.

Now, Spec $A$ is irreducible, and the points $x$ and $x^{\prime}$ of Spec $R_{\bar{\rho}}^{\mathbf{v}_{\lambda}, c r}$ corresponding to $\rho$ and $\rho^{\prime}$ respectively are in the image of the map $\operatorname{Spec} A \rightarrow \operatorname{Spec} R_{\bar{\rho}}^{\mathbf{v}_{\lambda}, c r}$, because they correspond to specialising the matrix $X$ to the matrices $1_{n}$ and $g$ respectively. The result follows.

Corollary 3.4.4. Let $M$ be a finite extension of $\mathbb{Q}_{l}$. Let $\bar{\rho}: G_{M} \rightarrow \mathrm{GL}_{n}(k)$ be the trivial representation, and let $\rho, \rho^{\prime}: G_{M} \rightarrow \mathrm{GL}_{n}(\mathcal{O})$ be two crystalline lifts of $\bar{\rho}$ which are both $\mathrm{GL}_{n}(\mathcal{O})$-conjugate to direct sums of unramified twists of a common set of crystalline characters. Then $\rho \sim \rho^{\prime}$.

Proof. After applying Lemma 3.4.3. we may assume that

$$
\rho=\bigoplus_{i=1}^{n} \rho_{i}
$$


and

$$
\rho^{\prime}=\bigoplus_{i=1}^{n} \rho_{i}^{\prime},
$$

where $\rho_{i}^{\prime}$ and $\rho_{i}$ are crystalline characters $G_{M} \rightarrow \mathcal{O}^{\times}$which differ by an unramified twist for each $i$ and reduce to the trivial character modulo $\mathfrak{m}_{\mathcal{O}}$. It suffices to check that the corresponding points $x$ and $x^{\prime}$ of $R_{\bar{\rho}}^{\mathbf{v}_{\lambda}, c r}$ are path-connected.

As in the proof of Lemma 3.4.2 the universal unramified $\mathcal{O}$-lifting ring of the trivial character $G_{M} \rightarrow k^{\times}$is given by $\mathcal{O}[[Y]]$ with the universal lifting $\chi^{\square}$ sending Frob $_{M}$ to $1+Y$. Taking $n$ copies of this character, we obtain a lifting

$$
\bigoplus_{i=1}^{n} \rho_{i} \otimes \chi_{i}^{\square}
$$

of $\bar{\rho}$ to $\mathcal{O}\left[\left[Y_{1}, \ldots, Y_{n}\right]\right]$, and thus obtain a continuous map $\operatorname{Spec} \mathcal{O}\left[\left[Y_{1}, \ldots, Y_{n}\right]\right] \rightarrow$ Spec $R_{\bar{\rho}}^{\mathbf{v}_{\lambda}, c r}$. Both $x$ and $x^{\prime}$ are in the image of this map, so the result follows.

The following is Lemma 3.4.3 of [Ger09] (recall that the ring $R_{\bar{\rho}}^{\triangle_{\lambda}, c r}$ is defined below Definition 3.3.4 and is universal for crystalline ordinary lifts of weight $\lambda$ ).

Lemma 3.4.5. Suppose $M$ is a finite extension of $\mathbb{Q}_{l}$ and $\bar{\rho}: G_{M} \rightarrow \mathrm{GL}_{n}(k)$ is the trivial representation. If the ring $R_{\bar{\rho}}^{\triangle_{\lambda}, c r}$ is non-zero, then it is irreducible.

Lemma 3.4.6. Let $M$ be a finite extension of $\mathbb{Q}_{p}$ for some prime $p$ and let $\bar{\rho}$ : $G_{M} \rightarrow \mathrm{GL}_{n}(k)$ be a continuous homomorphism. Let $\rho_{1}, \rho_{2}: G_{M} \rightarrow \mathrm{GL}_{n}(\mathcal{O})$ be two lifts of $\bar{\rho}$. If $p \neq l$, suppose that $\rho_{1} \rightsquigarrow \mathcal{O} \rho_{2}$ and $\rho_{2} \rightsquigarrow \mathcal{O} \rho_{1}$. (Equivalently, assume that for each minimal prime $\mathfrak{p}$ of $R^{\square}$ the quotient $R^{\square} / \mathfrak{p}$ is geometrically irreducible, and $\rho_{1}, \rho_{2}$ each correspond to closed points contained in a common irreducible component of Spec $R \square[\bar{\rho}[1 / l]$, and neither point is contained in any other irreducible component.) If $p=l$, assume that $\rho_{1} \sim \rho_{2}$. Let $\chi_{1}, \chi_{2}: G_{M} \rightarrow \mathcal{O}^{\times}$ be continuous characters with $\bar{\chi}_{1}=\bar{\chi}_{2}$ and $\left.\chi_{1}\right|_{I_{M}}=\left.\chi_{2}\right|_{I_{M}}$. Suppose in addition that if $p=l$, then $\chi_{1}$ and $\chi_{2}$ are crystalline. Then $\chi_{1} \rho_{1} \rightsquigarrow \mathcal{O} \chi_{2} \rho_{2}$ if $p \neq l$ and $\chi_{1} \rho_{1} \sim \chi_{2} \rho_{2}$ if $p=l$.

Proof. We treat the case $p \neq l$, the other case being similar. Let $\bar{\chi}=\bar{\chi}_{1}=\bar{\chi}_{2}$. Then the operation of twisting by $\chi_{1}$ defines an isomorphism of the lifting problems of $\bar{\rho}$ and $\overline{\chi \rho}$. It therefore defines an isomorphism $R_{\overline{\chi \rho}}^{\square} \stackrel{\sim}{\longrightarrow} R_{\bar{\rho}}^{\square}$. It follows that $\chi_{1} \rho_{1} \rightsquigarrow \mathcal{O} \quad \chi_{1} \rho_{2}$ and that $\chi_{1} \rho_{2}$ gives rise to a closed point of Spec $R \square \overline{\chi \rho}[1 / l]$ lying on a unique irreducible component. Since $\chi_{1}$ and $\chi_{2}$ differ by a residually trivial unramified twist, an easy argument shows that this component also contains $\chi_{2} \rho_{2}$ (cf. the proof of Corollary 3.4.4). It follows that $\chi_{1} \rho_{1} \rightsquigarrow \mathcal{O} \chi_{2} \rho_{2}$.

3.5. Global deformation rings. Let $F / F^{+}$be a totally imaginary quadratic extension of a totally real field $F^{+}$. Let $c$ denote the non-trivial element of $\operatorname{Gal}\left(F / F^{+}\right)$. Let $k$ denote a finite field of characteristic $l$ and $K$ a finite extension of $\mathbb{Q}_{l}$, inside our fixed algebraic closure $\overline{\mathbb{Q}}_{l}$, with ring of integers $\mathcal{O}$ and residue field $k$. Assume that $K$ contains the image of every embedding $F \hookrightarrow \overline{\mathbb{Q}}_{l}$ and that the prime $l$ is odd. Assume that every place in $F^{+}$dividing $l$ splits in $F$. Let $S$ denote a finite set of finite places of $F^{+}$which split in $F$, and assume that $S$ contains every place dividing $l$. Let $S_{l}$ denote the set of places of $F^{+}$lying over $l$. Let $F(S)$ denote the maximal extension of $F$ unramified away from $S$. Let $G_{F^{+}, S}=\operatorname{Gal}\left(F(S) / F^{+}\right)$ and $G_{F, S}=\operatorname{Gal}(F(S) / F)$. For each $v \in S$ choose a place $\widetilde{v}$ of $F$ lying over $v$ and 
let $\widetilde{S}$ denote the set of $\widetilde{v}$ for $v \in S$. For each place $v \mid \infty$ of $F^{+}$we let $c_{v}$ denote a choice of a complex conjugation at $v$ in $G_{F^{+}, S}$. For each place $w$ of $F$ we have a $G_{F, S}$-conjugacy class of homomorphisms $G_{F_{w}} \rightarrow G_{F, S}$. For $v \in S$ we fix a choice of homomorphism $G_{F_{\widetilde{v}}} \rightarrow G_{F, S}$.

Fix a continuous homomorphism

$$
\bar{r}: G_{F^{+}, S} \rightarrow \mathcal{G}_{n}(k)
$$

such that $G_{F, S}=\bar{r}^{-1}\left(\mathrm{GL}_{n}(k) \times \mathrm{GL}_{1}(k)\right)$ and fix a continuous character $\chi: G_{F^{+}, S} \rightarrow$ $\mathcal{O}^{\times}$such that $\nu \circ \bar{r}=\bar{\chi}$. Assume that $\left.\bar{r}\right|_{G_{F, S}}$ is absolutely irreducible. As in Definition 1.2.1 of CHT08, we define

- a lifting of $\bar{r}$ to an object $A$ of $\mathcal{C}_{\mathcal{O}}$ to be a continuous homomorphism $r: G_{F^{+}, S} \rightarrow \mathcal{G}_{n}(A)$ lifting $\bar{r}$ and with $\nu \circ r=\chi$;

- two liftings $r, r^{\prime}$ of $\bar{r}$ to $A$ to be equivalent if they are conjugate by an element of $\operatorname{ker}\left(\mathrm{GL}_{n}(A) \rightarrow \mathrm{GL}_{n}(k)\right)$;

- a deformation of $\bar{r}$ to an object $A$ of $\mathcal{C}_{\mathcal{O}}$ to be an equivalence class of liftings.

Similarly, if $T \subset S$, we define

- a $T$-framed lifting of $\bar{r}$ to $A$ to be a tuple $\left(r,\left\{\alpha_{v}\right\}_{v \in T}\right)$, where $r$ is a lifting of $\bar{r}$ and $\alpha_{v} \in \operatorname{ker}\left(\mathrm{GL}_{n}(A) \rightarrow \mathrm{GL}_{n}(k)\right)$ for $v \in T$;

- two $T$-framed liftings $\left(r,\left\{\alpha_{v}\right\}_{v \in T}\right),\left(r^{\prime},\left\{\alpha_{v}^{\prime}\right\}_{v \in T}\right)$ to be equivalent if there is an element $\beta \in \operatorname{ker}\left(\mathrm{GL}_{n}(A) \rightarrow \mathrm{GL}_{n}(k)\right)$ with $r^{\prime}=\beta r \beta^{-1}$ and $\alpha_{v}^{\prime}=\beta \alpha_{v}$ for $v \in T$;

- a $T$-framed deformation of $\bar{r}$ to be an equivalence class of $T$-framed liftings.

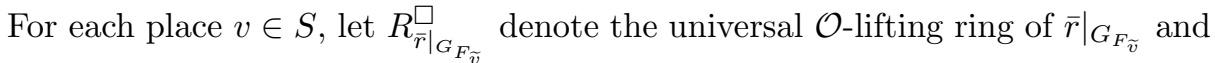
let $R_{\widetilde{v}}$ denote a quotient of $R_{\left.\bar{r}\right|_{G_{F}}}^{\square}$ which satisfies the following property:

(*) let $A$ be an object of $\mathcal{C}_{\mathcal{O}}$ and let $\zeta, \zeta^{\prime}:\left.R_{\widetilde{r}}^{\square}\right|_{G_{\widetilde{v}}} \rightarrow A$ be homomorphisms corresponding to two lifts $r$ and $r^{\prime}$ of $\left.\bar{r}\right|_{G_{F_{\tilde{r}}}}$ which are conjugate by an element of $\operatorname{ker}\left(\mathrm{GL}_{n}(A) \rightarrow \mathrm{GL}_{n}(k)\right)$. Then $\zeta$ factors through $R_{\widetilde{v}}$ if and only if $\zeta^{\prime}$ does.

We consider the deformation problem

$$
\mathcal{S}=\left(F / F^{+}, S, \widetilde{S}, \mathcal{O}, \bar{r}, \chi,\left\{R_{\widetilde{v}}\right\}_{v \in S}\right)
$$

(see Sections 2.2 and 2.3 of CHT08 for this terminology). We say that a lifting $r$ : $G_{F^{+}, S} \rightarrow \mathcal{G}_{n}(A)$ is of type $\mathcal{S}$ if for each place $v \in S$, the homomorphism $R_{\left.\bar{r}\right|_{G_{F}}} \rightarrow A$ corresponding to $\left.r\right|_{G_{F_{\widetilde{v}}}}$ factors through $R_{\widetilde{v}}$. We also define deformations of type $\mathcal{S}$ in the same way.

Let $\operatorname{Def}_{\mathcal{S}}$ be the functor $\mathcal{C}_{\mathcal{O}} \rightarrow$ Sets which sends an algebra $A$ to the set of deformations of $\bar{r}$ to $A$ of type $\mathcal{S}$. Similarly, if $T \subset S$, let $\operatorname{Def}_{\mathcal{S}}^{\square_{T}}$ be the functor $\mathcal{C}_{\mathcal{O}} \rightarrow$ Sets which sends an algebra $A$ to the set of $T$-framed liftings of $\bar{r}$ to $A$ which are of type $\mathcal{S}$. By Proposition 2.2.9 of [CHT08 these functors are represented by objects $R_{\mathcal{S}}^{\text {univ }}$ and $R_{\mathcal{S}}^{\square_{T}}$ respectively of $\mathcal{C}_{\mathcal{O}}$.

Lemma 3.5.1. Let $M$ be a finite extension of $\mathbb{Q}_{p}$ for some prime $p$. Let $\bar{\rho}: G_{M} \rightarrow$ $\mathrm{GL}_{n}(k)$ be a continuous homomorphism. If $p \neq l$, let $R$ be the maximal $l$-torsion free quotient of $R_{\bar{\rho}}^{\square}$. If $p=l$, assume that $K$ contains the image of each embedding $M \hookrightarrow \overline{\mathbb{Q}}_{l}$ and let $R=R_{\bar{\rho}}^{\mathbf{v}_{\lambda}, c r}$ for some $\lambda \in\left(\mathbb{Z}_{+}^{n}\right)^{\operatorname{Hom}(M, K)}$. Then $R$ satisfies property $(*)$ above. 
Proof. This can be proved in exactly the same way as Lemma 3.4.2.

\subsection{Automorphy lifting.}

\subsubsection{Fields.}

Theorem 3.6.1. Let $F$ be an imaginary $C M$ field with totally real subfield $F^{+}$and let $c$ be the non-trivial element of $\operatorname{Gal}\left(F / F^{+}\right)$. Let $n \in \mathbb{Z}_{\geq 1}$ and let $l>n$ be a prime. Let $K \subset \overline{\mathbb{Q}}_{l}$ denote a finite extension of $\mathbb{Q}_{l}$ with ring of integers $\mathcal{O}$ and residue field $k$. Assume that $K$ contains the image of every embedding $F \hookrightarrow \overline{\mathbb{Q}}_{l}$. Let

$$
\rho: G_{F} \rightarrow \mathrm{GL}_{n}(\mathcal{O})
$$

be a continuous representation and let $\bar{\rho}=\rho \bmod \mathfrak{m}_{\mathcal{O}}$. Suppose that $\rho$ enjoys the following properties:

(1) $\rho^{c} \cong \rho^{\vee} \epsilon^{1-n}$.

(2) The reduction $\bar{\rho}$ is absolutely irreducible and $\bar{\rho}\left(G_{F\left(\zeta_{l}\right)}\right) \subset \mathrm{GL}_{n}(k)$ is big (see Definition 4.1.1).

(3) $(\bar{F})^{\mathrm{ker} a d \bar{\rho}}$ does not contain $\zeta_{l}$.

(4) There is a continuous representation $\rho^{\prime}: G_{F} \rightarrow \mathrm{GL}_{n}(\mathcal{O})$, a RACSDC automorphic representation $\pi$ of $\mathrm{GL}_{n}\left(\mathbb{A}_{F}\right)$ which is unramified above $l$ and $\iota: \overline{\mathbb{Q}}_{l} \stackrel{\sim}{\longrightarrow} \mathbb{C}$ such that

(a) $\rho^{\prime} \otimes_{\mathcal{O}} \overline{\mathbb{Q}}_{l} \cong r_{l, L}(\pi): G_{F} \rightarrow \mathrm{GL}_{n}\left(\overline{\mathbb{Q}}_{l}\right)$.

(b) $\bar{\rho}=\bar{\rho}^{\prime}$.

(c) For all places $v \nmid l$ of $F$, either

- $\left.\rho\right|_{G_{F_{v}}}$ and $\pi_{v}$ are both unramified, or

- $\left.\left.\rho^{\prime}\right|_{G_{F_{v}}} \rightsquigarrow \mathcal{O} \rho\right|_{G_{F_{v}}}$.

(d) For all places $\left.v|l, \rho|_{G_{F_{v}}} \sim \rho^{\prime}\right|_{G_{F_{v}}}$.

Then $\rho$ is automorphic.

Proof. Choose a place $v_{1}$ of $F$ not dividing $l$ such that

- $v_{1}$ is unramified over a rational prime $p$ with $\left[F\left(\zeta_{p}\right): F\right]>n$;

- $v_{1}$ does not split completely in $F\left(\zeta_{l}\right)$;

- $\rho$ and $\pi$ are unramified at $v_{1}$;

- $\operatorname{ad} \bar{\rho}\left(\operatorname{Frob}_{v_{1}}\right)=1$.

Extending $\mathcal{O}$ if necessary, choose an imaginary $\mathrm{CM}$ field $L / F$ such that:

- $L / F$ is solvable.

- $L$ is linearly disjoint from $\bar{F}^{\mathrm{ker} \bar{r}}\left(\zeta_{l}\right)$ over $F$.

- $4 \mid\left[L^{+}: F^{+}\right]$, where $L^{+}$denotes the maximal totally real subfield of $L$.

- $L / L^{+}$is unramified at all finite places.

- Every prime of $L$ dividing $l$ is split over $L^{+}$and every prime where $\left.\rho\right|_{G_{L}}$ or $\pi_{L}$ ramifies is split over $L^{+}$(here $\pi_{L}$ denotes the base change of $\pi$ to $L$ ).

- Every place of $L$ over $v_{1}$ or $c v_{1}$ is split over $L^{+}$. Moreover, $v_{1}$ and $c v_{1}$ split completely in $L$.

- Every place $v \mid l$ of $F$ splits completely in $L$.

- If $v \nmid l$ is a place of $F$ and at least one of $\left.\rho\right|_{G_{F_{v}}}$ or $\pi_{v}$ is ramified, then $v$ splits completely in $L$.

Let $G / \mathcal{O}_{L^{+}}$be an algebraic group as in section 3.2 (with $F / F^{+}$replaced by $L / L^{+}$). By Théorème 5.4 and Corollaire 5.3 of Lab09 there exists an automorphic representation $\Pi$ of $G\left(\mathbb{A}_{L^{+}}\right)$such that $\pi_{L}$ is a strong base change of $\Pi$. Let $S_{l}$ 
denote the set of places of $L^{+}$dividing $l$ and let $R$ denote the set of places of $L^{+}$ not dividing $l$ and lying under a place of $L$ where $\rho$ or $\pi_{L}$ is ramified. Let $S_{a}$ denote the set of places of $L^{+}$lying over the restriction of $v_{1}$ to $F^{+}$. Let $T=S_{l} \sqcup R \sqcup S_{a}$. For each place $v \in T$, choose a place $\widetilde{v}$ of $L$ lying over it and let $\widetilde{T}$ denote the set of $\widetilde{v}$ for $v \in T$. Let $U=\prod_{v} U_{v} \subset G\left(\mathbb{A}_{L^{+}}^{\infty}\right)$ be a compact open subgroup such that

- $U_{v}=G\left(\mathcal{O}_{L_{v}^{+}}\right)$for $v \in S_{l}$ and for $v \notin T$ split in $L$;

- $U_{v}$ is a hyperspecial maximal compact subgroup of $G\left(L_{v}^{+}\right)$for each $v$ inert in $L$;

- $U_{v}$ is such that $\Pi_{v}^{U_{v}} \neq\{0\}$ for $v \in R$;

- $U_{v}=\operatorname{ker}\left(G\left(\mathcal{O}_{L_{v}^{+}}\right) \rightarrow G\left(k_{v}\right)\right)$ for $v \in S_{a}$.

Extend $K$ if necessary so that it contains the image of every embedding $L \hookrightarrow \overline{\mathbb{Q}}_{l}$. For each $v \in S_{l}$, let $\lambda_{\widetilde{v}}$ be the element of $\left(\mathbb{Z}_{+}^{n}\right)^{\operatorname{Hom}\left(L_{\widetilde{v}}, K\right)}$ with the property that $\left.\rho\right|_{G_{L_{\tilde{v}}}}$ and $\left.\rho^{\prime}\right|_{G_{L_{\tilde{v}}}}$ have $l$-adic Hodge type $\mathbf{v}_{\lambda_{\tilde{v}}}$. Let $\widetilde{I}_{l}$ denote the set of embeddings $L \hookrightarrow K$ giving rise to one of the places $\widetilde{v}$. Let $\lambda=\left(\lambda_{\widetilde{v}}\right)_{v \in S_{l}}$ regarded as an element of $\left(\mathbb{Z}_{+}^{n}\right)^{\widetilde{I}_{l}}$ in the evident way and let $S_{\lambda}(U, \mathcal{O})$ be the space of $l$-adic automorphic forms on $G$ of weight $\lambda$ introduced above. Let $\mathbb{T}_{\lambda}^{T}(U, \mathcal{O})$ be the $\mathcal{O}$-subalgebra of $\operatorname{End}_{\mathcal{O}}\left(S_{\lambda}(U, \mathcal{O})\right)$ generated by the Hecke operators $T_{w}^{(j)},\left(T_{w}^{(n)}\right)^{-1}$ for $w$ a place of $L$ split over $L^{+}$, not lying over $T$ and $j=1, \ldots, n$. The eigenvalues of the operators $T_{w}^{(j)}$ on the space $\left(\iota^{-1} \Pi^{\infty}\right)^{U}$ give rise to a homomorphism of $\mathcal{O}$-algebras $\mathbb{T}_{\lambda}^{T}(U, \mathcal{O}) \rightarrow \overline{\mathbb{Q}}_{l}$. Extending $K$ if necessary, we can and do assume that this homomorphism takes values in $\mathcal{O}$. Let $\mathfrak{m}$ denote the unique maximal ideal of $\mathbb{T}_{\lambda}^{T}(U, \mathcal{O})$ containing the kernel of this homomorphism.

Let $\delta_{L / L^{+}}$be the quadratic character of $G_{L^{+}}$corresponding to $L$. By Lemma 2.1.4 of CHT08 we can and do extend $\rho$ and $\rho^{\prime}$ to homomorphisms $r, r^{\prime}: G_{L^{+}} \rightarrow$ $\mathcal{G}_{n}(\mathcal{O})$ with $r \otimes k=r^{\prime} \otimes k: G_{L^{+}} \rightarrow \mathcal{G}_{n}(k),\left.r\right|_{G_{L}}=\left(\left.\rho\right|_{G_{L}}, \epsilon^{1-n}\right),\left.r^{\prime}\right|_{G_{L}}=\left(\left.\rho^{\prime}\right|_{G_{L}}, \epsilon^{1-n}\right)$ and $\nu \circ r=\nu \circ r^{\prime}=\epsilon^{1-n} \delta_{L / L^{+}}^{\mu}$ for some $\mu \in(\mathbb{Z} / 2 \mathbb{Z})$. Let $\bar{r}=r \otimes k: G_{L^{+}} \rightarrow \mathcal{G}_{n}(k)$.

For $v \in R \cup S_{a}$, let $\bar{R}_{\left.\bar{r}\right|_{G_{L}}}^{\square}$ denote the maximal $l$-torsion free quotient of $R_{\left.\bar{r}\right|_{G_{\tilde{v}}}}$. Note that $R_{\left.\bar{r}\right|_{G}}$ is formally smooth over $\mathcal{O}$ for $v \in S_{a}$ by Lemma 2.4.9 of [CHT08],

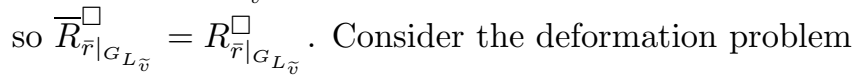

$$
\mathcal{S}:=\left(L / L^{+}, T, \widetilde{T}, \mathcal{O}, \bar{r}, \epsilon^{1-n} \delta_{L / L^{+}}^{\mu},\left\{R_{\left.\bar{r}\right|_{G_{L_{\tilde{v}}}} ^{\mathbf{v}_{\lambda_{\tilde{v}}}, c r}}^{\mu}\right\}_{v \in S_{l}} \cup\left\{\bar{R}_{\left.\bar{r}\right|_{G_{L}}}^{\square}\right\}_{v \in R \cup S_{a}}\right) .
$$

By Lemma 3.5.1, the rings $R_{\left.\bar{r}\right|_{G_{\tilde{V}}}}^{\mathbf{v}_{L_{\tilde{v}}}, c r}$ for $v \in S_{l}$ and $\bar{R}_{\left.\bar{r}\right|_{G_{\tilde{V}}}}^{\square}$ for $v \in R$ satisfy the property $(*)$ of Section 3.5 . Let $R_{\mathcal{S}}^{\text {univ }}$ be the object representing the corresponding deformation functor. Note that $\left.\bar{r}\right|_{G_{L}}$ is $\mathrm{GL}_{n}(k)$-conjugate to $\bar{r}_{\mathfrak{m}}$, where $\bar{r}_{\mathfrak{m}}$ is the representation associated to the maximal ideal $\mathfrak{m}$ of $\mathbb{T}_{\lambda}^{T}(U, \mathcal{O})$ in Section 3.2 . After conjugating we can and do assume that $\left.\bar{r}\right|_{G_{L}}=\bar{r}_{\mathfrak{m}}$. Since $\mathfrak{m}$ is non-Eisenstein, we have as above a continuous lift

$$
r_{\mathfrak{m}}: G_{L^{+}} \rightarrow \mathcal{G}_{n}\left(\mathbb{T}_{\lambda}^{T}(U, \mathcal{O})_{\mathfrak{m}}\right)
$$

of $\bar{r}$. Properties (0)-(3) of $r_{\mathfrak{m}}$ and the fact that $\mathbb{T}_{\lambda}^{T}(U, \mathcal{O})_{\mathfrak{m}}$ is $l$-torsion free and reduced imply that $r_{\mathfrak{m}}$ is of type $\mathcal{S}$. Hence $r_{\mathfrak{m}}$ gives rise to an $\mathcal{O}$-algebra homomorphism

$$
R_{\mathcal{S}}^{\text {univ }} \rightarrow \mathbb{T}_{\lambda}^{T}(U, \mathcal{O})_{\mathfrak{m}}
$$


(which is surjective by property (2) of $r_{\mathfrak{m}}$ ). To prove the theorem it suffices to show that the homomorphism $R_{\mathcal{S}}^{\text {univ }} \rightarrow \mathcal{O}$ corresponding to $r$ factors through $\mathbb{T}_{\lambda}^{T}(U, \mathcal{O})_{\mathfrak{m}}$.

We define

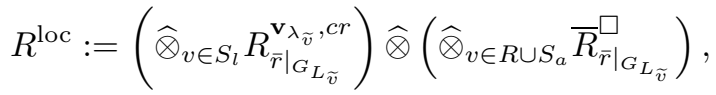

where all completed tensor products are taken over $\mathcal{O}$. Note that $R^{\text {loc }}$ is equidimensional of dimension $1+n^{2} \# T+\left[L^{+}: \mathbb{Q}\right] n(n-1) / 2$ by Lemma 3.3 of [BLGHT09].

Sublemma. There are integers $q, g \in \mathbb{Z}_{\geq 0}$ with

$$
1+q+n^{2} \# T=\operatorname{dim} R^{\text {loc }}+g
$$

and a module $M_{\infty}$ for both $R_{\infty}:=R^{\operatorname{loc}}\left[\left[x_{1}, \ldots, x_{g}\right]\right]$ and $S_{\infty}:=\mathcal{O}\left[\left[z_{1}, \ldots, z_{n^{2} \# T}\right.\right.$, $\left.\left.y_{1}, \ldots, y_{q}\right]\right]$ such that:

(1) $M_{\infty}$ is finite and free over $S_{\infty}$.

(2) $M_{\infty} /\left(z_{i}, y_{j}\right) \cong S_{\lambda}(U, \mathcal{O})_{\mathfrak{m}}$.

(3) The action of $S_{\infty}$ on $M_{\infty}$ can be factored through an $\mathcal{O}$-algebra homomorphism $S_{\infty} \rightarrow R_{\infty}$.

(4) There is a surjection $R_{\infty} \rightarrow R_{\mathcal{S}}^{\text {univ }}$ whose kernel contains all the $z_{i}$ and $y_{j}$ and which is compatible with the actions of $R_{\infty} /\left(z_{i}, y_{j}\right)$ and $R_{\mathcal{S}}^{\text {univ }}$ on $M_{\infty} /$ $\left(z_{i}, y_{j}\right) \cong S_{\lambda}(U, \mathcal{O})_{\mathfrak{m}}$. Moreover, there is a lift $r_{\mathcal{S}}^{\text {univ }}: G_{L^{+}} \rightarrow \mathcal{G}_{n}\left(R_{\mathcal{S}}^{\text {univ }}\right)$ of $\bar{r}$ representing the universal deformation so that for each $v \in T$, the composite $R_{\left.\bar{r}\right|_{G_{\tilde{v}}}}^{\square} \rightarrow R^{\text {loc }} \rightarrow R_{\infty} \rightarrow R_{\mathcal{S}}^{\text {univ }}$ arises from the lift $\left.r_{\mathcal{S}}^{\text {univ }}\right|_{G_{L_{\tilde{v}}}}$.

Assuming the sublemma for now, let us finish the proof of the theorem. Since $R_{\infty}$ is equidimensional of dimension $\operatorname{dim} R^{\text {loc }}+g$, it follows from (1) and (3) that the support of $M_{\infty}$ in $R_{\infty}$ is a union of irreducible components. (Indeed, by Lemma 2.3 of Tay08, it is enough to check that the $\mathfrak{m}_{R_{\infty}}$-depth of $M_{\infty}$ is equal to $\operatorname{dim} R_{\infty}=$ $\operatorname{dim} S_{\infty}$. By (3) it is enough to check the same statement for the $\mathfrak{m}_{S_{\infty}}$-depth, and this is immediate from (1).) The conjugacy class of $r^{\prime}$ determines a homomorphism $\zeta^{\prime}: R_{\mathcal{S}}^{\text {univ }} \rightarrow \mathcal{O}$ so that $r^{\prime}$ is $\operatorname{ker}\left(\mathrm{GL}_{n}(\mathcal{O}) \rightarrow \mathrm{GL}_{n}(k)\right)$-conjugate to $\zeta^{\prime} \circ r_{\mathcal{S}}^{\text {univ }}$. By the choice of $L$, for each $v \in R,\left.r^{\prime}\right|_{G_{L_{\tilde{v}}}}$ lies on a unique irreducible component $\mathcal{C}_{\widetilde{v}}$ of

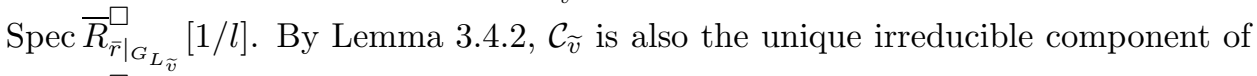

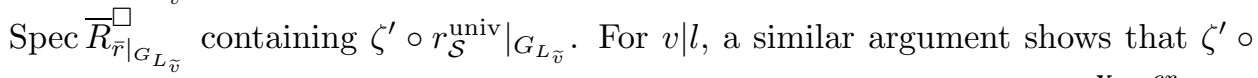

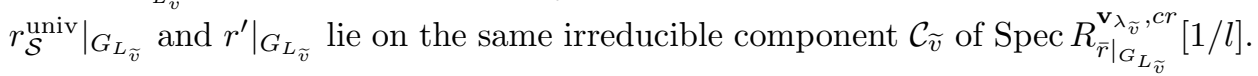

The conjugacy class of $r$ determines a homomorphism $\zeta: R_{\mathcal{S}}^{\text {univ }} \rightarrow \mathcal{O}$ so that $r$ is $\operatorname{ker}\left(\mathrm{GL}_{n}(\mathcal{O}) \rightarrow \mathrm{GL}_{n}(k)\right)$-conjugate to $\zeta \circ r_{\mathcal{S}}^{\text {univ }}$. By Lemma 3.4.2, the set of irreducible components of $\operatorname{Spec} \bar{R}_{\left.\bar{r}\right|_{G_{\tilde{v}}}}^{\square}[1 / l]$ containing $\left.\zeta \circ r_{\mathcal{S}}^{\text {univ }}\right|_{G_{L_{\tilde{v}}}}$ is equal to the set of components containing $\left.r\right|_{G_{L_{v}}}$. By the choice of $L$ it follows that $\mathcal{C}_{\widetilde{v}}$ contains $\left.\zeta \circ r_{\mathcal{S}}^{\text {univ }}\right|_{G_{L_{v}}}$. A similar argument, using part (d) of assumption (4) of the theorem, shows that $\mathcal{C}_{\widetilde{v}}$ contains $\left.\zeta \circ r_{\mathcal{S}}^{\text {univ }}\right|_{G_{L_{\tilde{v}}}}$ for $v \mid l$.

By part 5 of Lemma 3.3 of [BLGHT09], the irreducible components $\mathcal{C}_{\widetilde{v}}$ for $v \in$ $S_{l} \cup R$ determine an irreducible component $\mathcal{C}_{r^{\prime}}$ of Spec $R_{\infty}$ (as mentioned above, $R_{\left.\bar{r}\right|_{G_{L}}}$ is formally smooth over $\mathcal{O}$ for $v \in S_{a}$ ). Moreover, $\zeta^{\prime}$ composed with the surjection $R_{\infty} \rightarrow R_{\mathcal{S}}^{\text {univ }}$ of part (4) of the sublemma gives rise to a closed point of Spec $R_{\infty}[1 / l]$ which is in the support of $M_{\infty}$ and which lies in $\mathcal{C}_{r^{\prime}}$ but does not lie in any other irreducible component of $\operatorname{Spec} R_{\infty}$. We deduce that $\mathcal{C}_{r^{\prime}}$ is in the support 
of $M_{\infty}$. Since the closed point of $\operatorname{Spec} R_{\infty}[1 / l]$ corresponding to $\zeta$ lies in $\mathcal{C}_{r^{\prime}}$ it is also in the support of $M_{\infty}$ and we are done by assertion (4) of the sublemma.

Proof of sublemma. We apply the Taylor-Wiles-Kisin patching method. Let

$$
q_{0}=\left[L^{+}: \mathbb{Q}\right] n(n-1) / 2+\left[L^{+}: \mathbb{Q}\right] n\left(1-(-1)^{\mu-n}\right) / 2 .
$$

If $\left(Q, \widetilde{Q},\left\{\psi_{\widetilde{v}}\right\}_{v \in Q}\right)$ is a triple where

- $Q$ is a finite set of places of $L^{+}$disjoint from $T$ and consisting of places which split in $L$,

- $\widetilde{Q}$ consists of one place $\widetilde{v}$ of $L$ over each place $v \in Q$, and

- for each $v \in Q,\left.\bar{r}\right|_{G_{L_{\tilde{v}}}} \cong \bar{\psi}_{\widetilde{v}} \oplus \bar{s}_{\widetilde{v}}$, where $\operatorname{dim} \bar{\psi}_{\widetilde{v}}=1$ and $\bar{\psi}_{\widetilde{v}}$ is not isomorphic to any subquotient of $\bar{s} \widetilde{v}$,

then for each $v \in Q$, let $R_{\left.\bar{r}\right|_{G_{L}}}^{\bar{\psi}_{\widetilde{v}}}$ denote the quotient of $R_{\left.\bar{r}\right|_{G_{L}}}^{\square}$ corresponding to lifts $r: G_{L_{\tilde{v}}} \rightarrow \mathrm{GL}_{n}(A)$ which are $\operatorname{ker}\left(\mathrm{GL}_{n}(A) \rightarrow \mathrm{GL}_{n}(k)\right)$-conjugate to a lift of the form $\psi \oplus s$, where $\psi$ lifts $\bar{\psi}_{\widetilde{v}}$ and $s$ is an unramified lift of $\bar{s}_{\widetilde{v}}$. We then introduce the deformation problem

$$
\begin{aligned}
& \mathcal{S}_{Q}=\left(L / L^{+}, T \cup Q, \widetilde{T} \cup \widetilde{Q}, \mathcal{O}, \bar{r}, \epsilon^{1-n} \delta_{L / L^{+}}^{\mu},\right.
\end{aligned}
$$

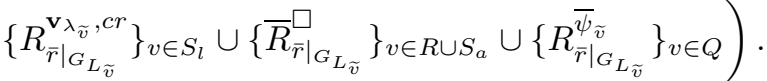

We define deformations (resp. $T$-framed deformations) of $\bar{r}$ of type $\mathcal{S}_{Q}$ in the evident manner and let $R_{\mathcal{S}_{Q}}^{\text {univ }}$ (resp. $R_{\mathcal{S}_{Q}}^{\square_{T}}$ ) denote the universal deformation ring (resp. $T$ framed deformation ring) of type $\mathcal{S}_{Q}$.

By Proposition 2.5.9 of [CHT08] we can and do choose an integer $q \geq q_{0}$ and for each $N \in \mathbb{Z}_{\geq 1}$ a tuple $\left(Q_{N}, \widetilde{Q}_{N},\left\{\psi_{\widetilde{v}}\right\}_{v \in Q_{N}}\right)$ as above with the following additional properties:

- $\# Q_{N}=q$ for all $N$;

- $\mathbf{N} v \equiv 1 \bmod l^{N}$ for $v \in Q_{N}$;

- the ring $R_{\mathcal{S}_{Q_{N}}}^{\square_{T}}$ can be topologically generated over $R^{\text {loc }}$ by

$$
q-q_{0}=q-\left[L^{+}: \mathbb{Q}\right] n(n-1) / 2-\left[L^{+}: \mathbb{Q}\right] n\left(1-(-1)^{\mu-n}\right) / 2
$$

elements.

For each $N \geq 1$, let $U_{1}\left(Q_{N}\right)=\prod_{v} U_{1}\left(Q_{N}\right)_{v}$ and $U_{0}\left(Q_{N}\right)=\prod_{v} U_{0}\left(Q_{N}\right)_{v}$ be the compact open subgroups of $G\left(\mathbb{A}_{L^{+}}^{\infty}\right)$ with $U_{i}\left(Q_{N}\right)_{v}=U_{v}$ for $v \notin Q_{N}, i=0,1$ and $U_{i}\left(Q_{N}\right)_{v}=\iota_{\widetilde{v}}^{-1} U_{i}(\widetilde{v})$ for $v \in Q_{N}, i=0,1$. Note that we have natural maps

$$
\mathbb{T}_{\lambda}^{T \cup Q_{N}}\left(U_{1}\left(Q_{N}\right), \mathcal{O}\right) \rightarrow \mathbb{T}_{\lambda}^{T \cup Q_{N}}\left(U_{0}\left(Q_{N}\right), \mathcal{O}\right) \rightarrow \mathbb{T}_{\lambda}^{T \cup Q_{N}}(U, \mathcal{O}) \hookrightarrow \mathbb{T}_{\lambda}^{T}(U, \mathcal{O}) .
$$

Thus $\mathfrak{m}$ determines maximal ideals of the first three algebras in this sequence which we denote by $\mathfrak{m}_{Q_{N}}$ for the first two and $\mathfrak{m}$ for the third. Note also that $\mathbb{T}_{\lambda}^{T \cup Q_{N}}(U, \mathcal{O})_{\mathfrak{m}}=\mathbb{T}_{\lambda}^{T}(U, \mathcal{O})_{\mathfrak{m}}$ by the proof of Corollary 3.4.5 of CHT08.

For each $v \in Q_{N}$ choose an element $\phi_{\widetilde{v}} \in G_{L_{\widetilde{v}}}$ lifting the geometric Frobenius and let $\varpi_{\widetilde{v}} \in \mathcal{O}_{L_{\widetilde{v}}}$ be the uniformiser with $\operatorname{Art}_{L_{\widetilde{v}}} \varpi_{\widetilde{v}}=\left.\phi_{\widetilde{v}}\right|_{L_{\widetilde{v}}^{\mathrm{ab}}}$. Let $P_{\widetilde{v}}(X) \in$ $\mathbb{T}_{\lambda}^{T \cup Q_{N}}\left(U_{1}\left(Q_{N}\right), \mathcal{O}\right)_{\mathfrak{m}_{Q_{N}}}[X]$ denote the characteristic polynomial of $r_{\mathfrak{m}_{Q_{N}}}\left(\phi_{\widetilde{v}}\right)$. By Hensel's lemma, we can factor $P_{\widetilde{v}}(X)=\left(X-A_{\widetilde{v}}\right) Q_{\widetilde{v}}(X)$, where $A_{\widetilde{v}}$ lifts $\bar{\psi}_{\widetilde{v}}\left(\phi_{\widetilde{v}}\right)$ 
and $Q_{\widetilde{v}}\left(A_{\widetilde{v}}\right)$ is a unit in $\mathbb{T}_{\lambda}^{T \cup Q_{N}}\left(U_{1}\left(Q_{N}\right), \mathcal{O}\right)_{\mathfrak{m}_{Q_{N}}}$. For $i=0,1$ and $\alpha \in L_{\widetilde{v}}$ of non-negative valuation, consider the Hecke operator

$$
V_{\alpha}:=\iota_{\widetilde{v}}^{-1}\left(\left[U_{i}(\widetilde{v})\left(\begin{array}{cc}
1_{n-1} & 0 \\
0 & \alpha
\end{array}\right) U_{i}(\widetilde{v})\right]\right)
$$

on $S_{\lambda}\left(U_{i}\left(Q_{N}\right), \mathcal{O}\right)_{\mathfrak{m}_{Q_{N}}}$. Let $G_{Q_{N}}=U_{0}\left(Q_{N}\right) / U_{1}\left(Q_{N}\right)$ and let $\Delta_{Q_{N}}$ denote the maximal $l$-power order quotient of $G_{Q_{N}}$. Let $\mathfrak{a}_{Q_{N}}$ denote the kernel of the augmentation $\operatorname{map} \mathcal{O}\left[\Delta_{Q_{N}}\right] \rightarrow \mathcal{O}$. For $i=0,1$, let

$$
H_{i, Q_{N}}=\prod_{v \in Q_{N}} Q_{\widetilde{v}}\left(V_{\varpi \widetilde{v}}\right) S_{\lambda}\left(U_{i}\left(Q_{N}\right), \mathcal{O}\right)_{\mathfrak{m}_{Q_{N}}}
$$

and let $\mathbb{T}_{i, Q_{N}}$ denote the image of $\mathbb{T}_{\lambda}^{T \cup Q_{N}}\left(U_{i}\left(Q_{N}\right), \mathcal{O}\right)$ in $\operatorname{End}_{\mathcal{O}}\left(H_{i, Q_{N}}\right)$. Let $H=$ $S_{\lambda}(U, \mathcal{O})_{\mathfrak{m}}$. We claim that the following hold:

(1) For each $N$, the map

$$
\prod_{v \in Q_{N}} Q_{\widetilde{v}}\left(V_{\varpi_{\tilde{v}}}\right): H \rightarrow H_{0, Q_{N}}
$$

is an isomorphism.

(2) For each $N, H_{1, Q_{N}}$ is free over $\mathcal{O}\left[\Delta_{Q_{N}}\right]$ with

$$
H_{1, Q_{N}} / \mathfrak{a}_{Q_{N}} \stackrel{\sim}{\longrightarrow} H_{0, Q_{N}} .
$$

(3) For each $N$ and each $v \in Q_{N}$, there is a character with open kernel $V_{\widetilde{v}}$ : $L_{\widetilde{v}}^{\times} \rightarrow \mathbb{T}_{1, Q_{N}}^{\times}$so that

(a) for each $\alpha \in L_{\widetilde{v}}$ of non-negative valuation, $V_{\alpha}=V_{\widetilde{v}}(\alpha)$ on $H_{1, Q_{N}}$;

(b) $\left.\left(r_{\mathfrak{m}_{Q_{N}}} \otimes \mathbb{T}_{1, Q_{N}}\right)\right|_{W_{L_{\widetilde{v}}}} \cong s \oplus\left(V_{\widetilde{v}} \circ \operatorname{Art}_{L_{\widetilde{v}}}^{-1}\right)$ with $s$ unramified, lifting $\bar{s}_{\widetilde{v}}$ and $\left(V_{\widetilde{v}} \circ \operatorname{Art}_{L_{\widetilde{v}}}^{-1}\right)$ lifting $\bar{\psi}_{\widetilde{v}}$.

To see this, note that Lemmas 3.1 .3 and 3.1.5 of [CHT08] imply that $P_{\widetilde{v}}\left(V_{\varpi_{\widetilde{v}}}\right)=0$ on $S_{\lambda}\left(U_{1}\left(Q_{N}\right), \mathcal{O}\right)_{\mathfrak{m}_{Q_{N}}}$. Property (1) now follows from Lemma 3.2.2 of CHT08 together with Lemma 3.1.5 of [CHT08] and the fact that $\mathbb{T}_{\lambda}^{T \cup Q_{N}}(U, \mathcal{O})_{\mathfrak{m}}=\mathbb{T}_{\lambda}^{T}(U, \mathcal{O})_{\mathfrak{m}}$. Property (3) follows exactly as in the proof of part 8 of Proposition 3.4 .4 of CHT08]. Note that $H_{1, Q_{N}}$ is a $\mathbb{T}_{\lambda}^{T}\left(U_{1}\left(Q_{N}\right), \mathcal{O}\right)_{\mathfrak{m}_{Q_{N}}}\left[G_{Q_{N}}\right]$-direct summand of $S_{\lambda}\left(U_{1}\left(Q_{N}\right), \mathcal{O}\right)_{\mathfrak{m}_{Q_{N}}}$. Moreover, it follows from the fact that $U$ is sufficiently small (see Lemma 3.3.1 of [CHT08]) that $S_{\lambda}\left(U_{1}\left(Q_{N}\right), \mathcal{O}\right)_{\mathfrak{m}_{Q_{N}}}$ is finite free over $\mathcal{O}\left[G_{Q_{N}}\right]$ with $G_{Q_{N}}$-coinvariants isomorphic to $S_{\lambda}\left(U_{0}\left(Q_{N}\right), \mathcal{O}\right)_{\mathfrak{m}_{Q_{N}}}$ via the trace map $\operatorname{tr}_{G_{Q_{N}}}$. It follows that $H_{1, Q_{N}}$ has $G_{Q_{N}}$-coinvariants isomorphic to $H_{0, Q_{N}}$ via $\operatorname{tr}_{G_{Q_{N}}}$. Finally, note that by $(3)$ the action of $\alpha=\left(\alpha_{\widetilde{v}}\right)_{v \in Q_{N}} \in G_{Q_{N}}$ on $H_{1, Q_{N}}$ is given by $\prod_{v \in Q_{N}} V_{\widetilde{v}}\left(\alpha_{\widetilde{v}}\right)$. Since each $\bar{\psi}_{\widetilde{v}}$ is unramified, the action of $G_{Q_{N}}$ on $H_{1, Q_{N}}$ must factor through $\Delta_{Q_{N}}$ and (2) follows.

For each $N$, the lift $r_{\mathfrak{m}_{Q_{N}}} \otimes \mathbb{T}_{1, Q_{N}}$ of $\bar{r}$ is of type $\mathcal{S}_{Q_{N}}$ and gives rise to a surjection $R_{\mathcal{S}_{Q_{N}}}^{\text {univ }} \rightarrow \mathbb{T}_{1, Q_{N}}$. Thinking of $\Delta_{Q_{N}}$ as the maximal l-power quotient of $\prod_{v \in Q_{N}} I_{L_{\tilde{v}}}$, the determinant of any choice of universal deformation $r_{\mathcal{S}_{Q_{N}}}^{\text {univ }}$ gives rise to a homomorphism $\Delta_{Q_{N}} \rightarrow\left(R_{\mathcal{S}_{Q_{N}}}^{\text {univ }}\right)^{\times}$. We thus have homomorphisms $\mathcal{O}\left[\Delta_{Q_{N}}\right] \rightarrow$

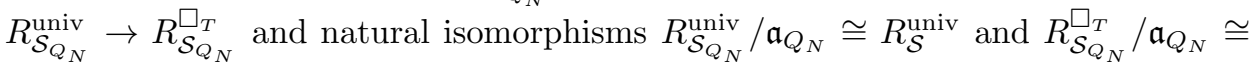
$R_{\mathcal{S}}^{\square_{T}}$.

Let

$$
\mathcal{T}=\mathcal{O}\left[\left[X_{v, i, j}: v \in T, i, j=1, \ldots, n\right]\right]
$$


Choose a lift $r_{\mathcal{S}}^{\text {univ }}: G_{L^{+}} \rightarrow \mathcal{G}_{n}\left(R_{\mathcal{S}}^{\text {univ }}\right)$ representing the universal deformation. The tuple $\left(r_{\mathcal{S}}^{\text {univ }},\left(1_{n}+X_{v, i, j}\right)_{v \in T}\right)$ gives rise to an isomorphism

$$
R_{\mathcal{S}}^{\square_{T}} \stackrel{\sim}{\longrightarrow} R_{\mathcal{S}}^{\text {univ }} \widehat{\otimes}_{\mathcal{O}} \mathcal{T}
$$

For each $N$, choose a lift $r_{\mathcal{S}_{Q_{N}}}^{\text {univ }}: G_{L^{+}} \rightarrow \mathcal{G}_{n}\left(R_{\mathcal{S}_{Q_{N}}}^{\text {univ }}\right)$ representing the universal deformation with $r_{S_{Q_{N}}}^{\text {univ }} \bmod \mathfrak{a}_{Q_{N}}=r_{\mathcal{S}}^{\text {univ }}$. This gives rise to an isomorphism $R_{\mathcal{S}_{Q_{N}}}^{\square_{T}} \stackrel{\sim}{\longrightarrow}$ $R_{\mathcal{S}_{Q_{N}}}^{\text {univ }} \widehat{\otimes}_{\mathcal{O}} \mathcal{T}$ which reduces modulo $\mathfrak{a}_{Q_{N}}$ to the isomorphism $R_{\mathcal{S}}^{\square_{T}} \stackrel{\sim}{\longrightarrow} R_{\mathcal{S}}^{\text {univ }} \widehat{\otimes}_{\mathcal{O}} \mathcal{T}$. We let

$$
\begin{aligned}
H^{\square} & =H \otimes_{R_{\mathcal{S}}^{\text {univ }}} R_{\mathcal{S}}^{\square}, \\
H_{1, Q_{N}}^{\square} & =H_{1, Q_{N}} \otimes_{R_{\mathcal{S}_{Q_{N}}}^{\text {univ }}} R_{\mathcal{S}_{Q_{N}}}^{\square}, \\
\mathbb{T}_{1, Q_{N}}^{\square_{T}} & =\mathbb{T}_{1, Q_{N}} \otimes_{R_{\mathcal{S}_{Q_{N}}}^{\text {univ }}} R_{\mathcal{S}_{Q_{N}}}^{\square_{T}} .
\end{aligned}
$$

Then $H_{1, Q_{N}}^{\square_{T}}$ is a finite free $\mathcal{T}\left[\Delta_{Q_{N}}\right]$-module with $H_{1, Q_{N}}^{\square_{T}} / \mathfrak{a}_{Q_{N}} \cong H^{\square_{T}}$, compatible with the isomorphism $R_{\mathcal{S}_{Q_{N}}}^{\square_{T}} / \mathfrak{a}_{Q_{N}} \cong R_{\mathcal{S}}^{\square}$.

Let $g=q-q_{0}$ and let

$$
\begin{aligned}
\Delta_{\infty} & =\mathbb{Z}_{l}^{q}, \\
R_{\infty} & =R^{\text {loc }}\left[\left[x_{1}, \ldots, x_{g}\right]\right], \\
S_{\infty} & =\mathcal{T}\left[\left[\Delta_{\infty}\right]\right]
\end{aligned}
$$

and let $\mathfrak{a}$ denote the kernel of the $\mathcal{O}$-algebra homomorphism $S_{\infty} \rightarrow \mathcal{O}$ which sends each $X_{v, i, j}$ to 0 and each element of $\Delta_{\infty}$ to 1 . Note that $S_{\infty}$ is formally smooth over $\mathcal{O}$ of relative dimension $q+n^{2} \# T$. For each $N$, choose a surjection $\Delta_{\infty} \rightarrow \Delta_{Q_{N}}$ and let $\mathfrak{c}_{N}$ denote the kernel of the corresponding homomorphism $S_{\infty} \rightarrow \mathcal{T}\left[\Delta_{Q_{N}}\right]$. For each $N \geq 1$, choose a surjection of $R^{\text {loc }}$-algebras

$$
R_{\infty} \rightarrow R_{\mathcal{S}_{Q_{N}}}^{\square} .
$$

We regard each $R_{\mathcal{S}_{Q_{N}}}^{\square_{T}}$ as an $S_{\infty}$-algebra via $S_{\infty} \rightarrow \mathcal{T}\left[\Delta_{Q_{N}}\right] \rightarrow R_{\mathcal{S}_{Q_{N}}}^{\square_{T}}$. In particular, $R_{\mathcal{S}_{Q_{N}}}^{\square_{T}} / \mathfrak{a} \cong R_{\mathcal{S}}^{\text {univ }}$

Choose a sequence of open ideals $\left(\mathfrak{b}_{N}\right)_{N \geq 1}$ of $S_{\infty}$ with

- $\mathfrak{b}_{N} \supset \mathfrak{c}_{N}$

- $\mathfrak{b}_{N} \supset \mathfrak{b}_{N+1}$, and

- $\bigcap_{N} \mathfrak{b}_{N}=(0)$.

Let $\mathbb{T}=\mathbb{T}_{\lambda}(U, \mathcal{O})_{\mathfrak{m}}$. Choose a sequence of open ideals $\left(\mathfrak{d}_{N}\right)_{N \geq 1}$ of $R_{\mathcal{S}}^{\text {univ }}$ with the following properties:

- $\mathfrak{b}_{N} R_{\mathcal{S}}^{\text {univ }}+\operatorname{ker}\left(R_{\mathcal{S}}^{\text {univ }} \rightarrow \mathbb{T}\right) \supset \mathfrak{d}_{N} \supset \mathfrak{b}_{N} R_{\mathcal{S}}^{\text {univ }}$

- $\mathfrak{d}_{N} \supset \mathfrak{d}_{N+1}$, and

- $\bigcap_{N} \mathfrak{d}_{N}=(0)$.

In the first bullet point, $S_{\infty}$ acts on $R_{\mathcal{S}}^{\text {univ }}$ via the quotient $S_{\infty} / \mathfrak{a}=\mathcal{O}$. In what follows, we also consider $S_{\infty}$ acting on $\mathbb{T}$ and $H$ via the quotient $S_{\infty} / \mathfrak{a}$.

For each $N \geq 1$, define a 'patching datum of level $N$ ' to consist of a tuple $(\phi, \mathcal{M}, \psi)$, where

- $\phi$ is a surjective homomorphism of $\mathcal{O}$-algebras

$$
\phi: R_{\infty} \rightarrow R_{\mathcal{S}}^{\text {univ }} / \mathfrak{d}_{N}
$$


- $\mathcal{M}$ is a module over $R_{\infty} \widehat{\otimes}_{\mathcal{O}} S_{\infty}$ which is finite free over $S_{\infty} / \mathfrak{b}_{N}$, and

- $\psi$ is an isomorphism of $\mathcal{O}$-modules

$$
\psi: \mathcal{M} / \mathfrak{a} \stackrel{\sim}{\longrightarrow} H / \mathfrak{b}_{N}
$$

compatible with the action of $R_{\infty}$ on $\mathcal{M} / \mathfrak{a}$, the action of $R_{\mathcal{S}}^{\text {univ }} / \mathfrak{d}_{N}$ on $H / \mathfrak{b}_{N}$ (via $R_{\mathcal{S}}^{\text {univ }} / \mathfrak{d}_{N} \rightarrow \mathbb{T} / \mathfrak{b}_{N}$ ) and the homomorphism $\phi$.

We consider two such patching data of level $N,(\phi, \mathcal{M}, \psi)$ and $\left(\phi^{\prime}, \mathcal{M}^{\prime}, \psi^{\prime}\right)$, to be equivalent if $\phi=\phi^{\prime}$ and there is an isomorphism $\mathcal{M} \cong \mathcal{M}^{\prime}$ of $R_{\infty} \widehat{\otimes}_{\mathcal{O}} S_{\infty}$-modules which is compatible with $\psi$ and $\psi^{\prime}$ when reduced modulo a. Note that there are only finitely many patching data of level $N$ up to equivalence. Note also that given $N^{\prime} \geq N \geq 1$ and a patching datum of level $N^{\prime}$, we can obtain a patching datum of level $N$ in an obvious fashion.

For each $M \geq N \geq 1$, let $D(M, N)$ be the patching datum of level $N$ consisting of

- the surjective homomorphism

$$
R_{\infty} \rightarrow R_{\mathcal{S}_{Q_{M}}}^{\square} \rightarrow R_{\mathcal{S}_{Q_{M}}}^{\square} / \mathfrak{a}=R_{\mathcal{S}}^{\text {univ }} \rightarrow R_{\mathcal{S}}^{\text {univ }} / \mathfrak{d}_{N}
$$

- the module $H_{1, Q_{M}}^{\square_{T}} / \mathfrak{b}_{N}$ which is finite free over $S_{\infty} / \mathfrak{b}_{N}$ and acted upon by $R_{\infty}$ via $R_{\infty} \rightarrow R_{\mathcal{S}_{Q_{M}}}^{\square_{T}} \rightarrow \mathbb{T}_{1, Q_{M}}^{\square}$, and

- the isomorphism

$$
H_{1, Q_{M}}^{\square_{T}} /\left(\mathfrak{a}+\mathfrak{b}_{N}\right) \cong H / \mathfrak{b}_{N}
$$

which is compatible with the homomorphism $R_{\infty} \rightarrow R_{\mathcal{S}_{Q_{M}}}^{\square_{T}} \rightarrow R_{\mathcal{S}}^{\text {univ }} / \mathfrak{d}_{N}$.

Since there are only finitely many patching data of each level $N$, we can and do choose a sequence of pairs of integers $\left(M_{i}, N_{i}\right)_{i \geq 1}$ with $M_{i+1}>M_{i}, N_{i+1}>N_{i}$ and $M_{i} \geq N_{i}$ for all $i$ such that $D\left(M_{i+1}, N_{i+1}\right)$ reduced to level $N_{i}$ is equivalent to $D\left(M_{i}, N_{i}\right)$. In other words,

- for each $i$, the homomorphism

$$
\phi_{i+1}: R_{\infty} \rightarrow R_{\mathcal{S}_{Q_{M_{i+1}}}}^{\square_{T}} \rightarrow R_{\mathcal{S}_{Q_{M_{i+1}}}}^{\square_{T}} / \mathfrak{a}=R_{\mathcal{S}}^{\text {univ }} \rightarrow R_{\mathcal{S}}^{\text {univ }} / \mathfrak{d}_{N_{i+1}},
$$

when reduced modulo $\mathfrak{d}_{N_{i}}$ is equal to the homomorphism

$$
\phi_{i}: R_{\infty} \rightarrow R_{\mathcal{S}_{Q_{M_{i}}}}^{\square_{T}} \rightarrow R_{\mathcal{S}_{Q_{M_{i}}}}^{\square_{T}} / \mathfrak{a}=R_{\mathcal{S}}^{\text {univ }} \rightarrow R_{\mathcal{S}}^{\text {univ }} / \mathfrak{d}_{N_{i}} ;
$$

- for each $i$, we can and do choose an isomorphism of $R_{\infty} \widehat{\otimes}_{\mathcal{O}} S_{\infty}$-modules

$$
\gamma_{i}: H_{1, Q_{M_{i+1}}}^{\square_{T}} / \mathfrak{b}_{N_{i}} \cong H_{1, Q_{M_{i}}}^{\square_{T}} / \mathfrak{b}_{N_{i}}
$$

Taking the inverse limit of the $\phi_{i}$ gives rise to a surjection

$$
R_{\infty} \rightarrow R_{\mathcal{S}}^{\text {univ }}
$$

Define

$$
H_{\infty}^{\square_{T}}:={\underset{l}{\leftarrow}}_{i} H_{1, Q_{M_{i}}}^{\square_{T}} / \mathfrak{b}_{N_{i}}
$$

where the limit is taken with respect to the $\gamma_{i}$. Then $H_{\infty}^{\square_{T}}$ is a module for $R_{\infty} \widehat{\otimes}_{\mathcal{O}} S_{\infty}$ which is finite free over $S_{\infty}$. Note that the image of $S_{\infty}$ in $\operatorname{End}_{S_{\infty}}\left(H_{\infty}^{\square_{T}}\right)$ is contained in the image of $R_{\infty}$ (indeed, the image of $R_{\infty}$ is closed and the corresponding statement is true for each $\left.H_{1, Q_{M_{i}}}^{\square_{T}} / b_{N_{i}}\right)$. Since $S_{\infty}$ is formally smooth over $\mathcal{O}$, we can and do factor the action of $S_{\infty}$ on $H_{\infty}^{\square_{T}}$ through $R_{\infty}$ (note that it suffices to 
define the factorisation on a set of topological generators of $S_{\infty}$ over $\mathcal{O}$ ). Note that we have

$$
H_{\infty}^{\square_{T}} / \mathfrak{a} \cong H
$$

compatible with the surjection $R_{\infty} \rightarrow R_{\mathcal{S}}^{\text {univ }}$. Since $R_{\infty}$ is equidimensional of dimension

$1+n^{2} \# T+\left[L^{+}: \mathbb{Q}\right] n(n-1) / 2+q-q_{0}=1+q+n^{2} \# T-\left[L^{+}: \mathbb{Q}\right] n\left(1-(-1)^{\mu-n}\right) / 2$

and $H_{\infty}^{\square_{T}}$ has $\mathfrak{m}_{R_{\infty}}$-depth at least

$$
1+q+n^{2} \# T
$$

(the dimension of $S_{\infty}$ ) we deduce from Lemma 2.3 of Tay08 that $\mu \equiv n \bmod 2$. Hence

$$
1+q+n^{2} \# T=\operatorname{dim} R^{\text {loc }}+g
$$

and taking $M_{\infty}=H_{\infty}^{\square_{T}}$, the sublemma is proved.

Since proving the sublemma was our only remaining task, the automorphy lifting theorem is proven.

\subsubsection{Totally real fields.}

Theorem 3.6.2. Let $F^{+}$be a totally real field. Let $l>n$ be a prime and let $K \subset \overline{\mathbb{Q}}_{l}$ denote a finite extension of $\mathbb{Q}_{l}$ with ring of integers $\mathcal{O}$ and residue field $k$. Assume that $K$ contains the image of every embedding $F^{+} \hookrightarrow \overline{\mathbb{Q}}_{l}$. Let

$$
\rho: G_{F^{+}} \rightarrow \mathrm{GL}_{n}(\mathcal{O})
$$

be a continuous representation and let $\bar{\rho}=\rho \bmod \mathfrak{m}_{\mathcal{O}}$. Suppose that $\rho$ enjoys the following properties:

(1) $\rho^{\vee} \cong \rho \epsilon^{n-1} \chi$ for some character $\chi: G_{F^{+}} \rightarrow \mathcal{O}^{\times}$with $\chi\left(c_{v}\right)$ independent of $v \mid \infty$ (where $c_{v}$ denotes a complex conjugation at $v$ ).

(2) The reduction $\bar{\rho}$ is absolutely irreducible and $\bar{\rho}\left(G_{F^{+}\left(\zeta_{l}\right)}\right) \subset \mathrm{GL}_{n}(k)$ is big (see Definition 4.1.1).

(3) $\left(\overline{F^{+}}\right)^{\text {ker ad } \bar{\rho}}$ does not contain $\zeta_{l}$.

(4) There is a continuous representation $\rho^{\prime}: G_{F^{+}} \rightarrow \mathrm{GL}_{n}(\mathcal{O})$, a RAESDC automorphic representation $\pi$ of $\mathrm{GL}_{n}\left(\mathbb{A}_{F^{+}}\right)$which is unramified above $l$ and $\iota: \overline{\mathbb{Q}}_{l} \stackrel{\sim}{\longrightarrow} \mathbb{C}$ such that:

(a) $\rho^{\prime} \otimes_{\mathcal{O}} \overline{\mathbb{Q}}_{l} \cong r_{l, l}(\pi): G_{F^{+}} \rightarrow \mathrm{GL}_{n}\left(\overline{\mathbb{Q}}_{l}\right)$ and $\left(\rho^{\prime}\right)^{\vee} \cong \rho^{\prime} \epsilon^{n-1} \chi^{\prime}$ for some character $\chi^{\prime}: G_{F^{+}} \rightarrow \mathcal{O}^{\times}$with $\bar{\chi}^{\prime}=\bar{\chi}$.

(b) $\bar{\rho}=\bar{\rho}^{\prime}$.

(c) For all places $v \nmid l$ of $F^{+}$, either $\left.\rho\right|_{G_{F_{v}^{+}}}$and $\pi_{v}$ are both unramified, or the following both hold:

- $\left.\left.\rho^{\prime}\right|_{G_{F_{v}^{+}}} \rightsquigarrow \mathcal{O} \rho\right|_{G_{F_{v}^{+}}}$and $\left.\left.\rho\right|_{G_{F_{v}^{+}}} \rightsquigarrow \mathcal{O} \rho^{\prime}\right|_{G_{F_{v}^{+}}}$.

- $\left.\chi\right|_{I_{F_{v}^{+}}}=\left.\chi^{\prime}\right|_{I_{F_{v}^{+}}}$.

(d) For all places $\left.v|l, \rho|_{G_{F_{v}}} \sim \rho^{\prime}\right|_{G_{F_{v}}}$.

Then $\rho$ is automorphic.

Proof. Extending $\mathcal{O}$ if necessary, choose a quadratic $\mathrm{CM}$ extension $F$ of $F^{+}$and algebraic characters $\psi, \psi^{\prime}: G_{F} \rightarrow \mathcal{O}^{\times}$such that the following hold:

(i) $F$ is linearly disjoint from ${\overline{F^{+}}}^{\operatorname{ker} \bar{r}}\left(\zeta_{l}\right)$ over $F^{+}$. 
(ii) Each place of $F^{+}$lying over $l$ and each place at which $\rho$ or $\pi$ is ramified splits completely in $F$.

(iii) $\psi \psi^{c}=\left.\chi\right|_{G_{F}}$.

(iv) $\psi$ and $\psi^{\prime}$ are crystalline above $l$.

(v) $\psi^{\prime}\left(\psi^{\prime}\right)^{c}=\left.\chi^{\prime}\right|_{G_{F}}$.

(vi) Let $S$ denote the set of places of $F$ which divide $l$ or which lie over a place of $F^{+}$where $\rho$ or $\pi$ ramifies. Then for all $w \in S$, we have $\left.\psi^{\prime}\right|_{I_{L_{w}}}=\left.\psi\right|_{I_{L_{w}}}$.

(vii) $\bar{\psi}=\bar{\psi}^{\prime}$.

(Take $F$ to be a quadratic CM extension of $F^{+}$satisfying (i) and (ii). Use Lemma 4.1.5 of CHT08 to construct a $\psi$ which satisfies (iii) and (iv). Note that $\chi$ and $\chi^{\prime}$ are crystalline characters of $G_{F^{+}}$with the same Hodge-Tate weights. In particular, for each place $v \mid l$ of $F^{+}$we have $\left.\chi\right|_{F_{F_{v}^{+}}}=\left.\chi^{\prime}\right|_{I_{F_{v}^{+}}}$. We can therefore apply Lemma 4.1.6 of [CHT08] to find a $\psi^{\prime}$ satisfying (v), (vi) and (vii).)

Let $r=\left.\psi \rho\right|_{G_{F}}$ and $r^{\prime}=\left.\psi^{\prime} \rho^{\prime}\right|_{G_{F}}$. Then $\bar{r}=\bar{r}^{\prime}, r^{c} \cong r^{\vee} \epsilon^{1-n}$ and $\left(r^{\prime}\right)^{c} \cong$ $\left(r^{\prime}\right)^{\vee} \epsilon^{1-n}$. For $w \in S$, Lemma 3.4.6 implies that $\left.\left.r\right|_{G_{F_{w}}} \sim r^{\prime}\right|_{G_{F_{w}}}$ if $v \mid l$ and both $\left.\left.r\right|_{G_{F_{w}}} \rightsquigarrow \mathcal{O} r^{\prime}\right|_{G_{F_{w}}}$ and $\left.\left.r^{\prime}\right|_{G_{F_{w}}} \rightsquigarrow \mathcal{O} r\right|_{G_{F_{w}}}$ otherwise.

The theorem now follows from Theorem 3.6.1 applied to $\left.r\right|_{G_{F}}$ and $\left.r^{\prime}\right|_{G_{F}}$, together with Lemma 1.5 of [BLGHT09].

3.6.3. Finiteness of a deformation ring. We now deduce a result on the finiteness of a universal deformation ring. This result is not needed for the main theorem, so this section may be skipped by readers interested only in the Sato-Tate conjecture. However, we believe that it is of independent interest, and it will prove useful to us in future work.

Let $F$ be an imaginary $\mathrm{CM}$ field with totally real subfield $F^{+}$and let $c$ be the non-trivial element of $\operatorname{Gal}\left(F / F^{+}\right)$. Let $n \in \mathbb{Z}_{\geq 1}$ and let $l>n$ be a prime. Let $K \subset \overline{\mathbb{Q}}_{l}$ denote a finite extension of $\mathbb{Q}_{l}$ with ring of integers $\mathcal{O}$ and residue field $k$. Assume that $K$ contains the image of every embedding $F \hookrightarrow \overline{\mathbb{Q}}_{l}$. Suppose in addition that each place of $F^{+}$dividing $l$ splits in $F$. Let

$$
\bar{\rho}: G_{F} \rightarrow \mathrm{GL}_{n}(k)
$$

be a continuous homomorphism and suppose

$$
\rho^{\prime}: G_{F} \rightarrow \mathrm{GL}_{n}(\mathcal{O})
$$

is a continuous lift of $\bar{\rho}$ which is automorphic of level prime to $l$. In particular, $\left(\rho^{\prime}\right)^{c} \cong\left(\rho^{\prime}\right)^{\vee} \epsilon^{1-n}$. By definition, there is an RACSDC automorphic representation $\pi$ of $\mathrm{GL}_{n}\left(\mathbb{A}_{F}\right)$ (which is unramified above $l$ ) and an isomorphism $\iota: \overline{\mathbb{Q}}_{l} \stackrel{\sim}{\longrightarrow} \mathbb{C}$ such that $\rho^{\prime} \otimes \overline{\mathbb{Q}}_{l} \cong r_{l, \iota}(\pi): G_{F} \rightarrow \mathrm{GL}_{n}\left(\overline{\mathbb{Q}}_{l}\right)$. Suppose that every finite place of $F$ at which $\pi$ is ramified is split over $F^{+}$. Let $S_{l}$ denote the set of places of $F^{+}$ lying above $l$. Let $R$ denote a finite set of finite places of $F^{+}$disjoint from $S_{l}$ and containing the restriction to $F^{+}$of every finite place of $F$ where $\pi$ is ramified.

Let $\delta_{F / F^{+}}$be the quadratic character of $G_{F^{+}}$corresponding to $F$. By Lemma 2.1.4 of [CHT08] we can and do extend $\bar{\rho}$ and $\rho^{\prime}$ to homomorphisms $\bar{r}: G_{F^{+}} \rightarrow$ $\mathcal{G}_{n}(k)$ and $r^{\prime}: G_{F^{+}} \rightarrow \mathcal{G}_{n}(\mathcal{O})$ with $r^{\prime} \otimes k=\bar{r},\left.\bar{r}\right|_{G_{F}}=\left(\bar{\rho}, \epsilon^{1-n}\right),\left.r^{\prime}\right|_{G_{F}}=\left(\left.\rho^{\prime}\right|_{G_{F}}, \epsilon^{1-n}\right)$ and $\nu \circ r^{\prime}=\epsilon^{1-n} \delta_{F / F^{+}}^{\mu}$ for some $\mu \in(\mathbb{Z} / 2 \mathbb{Z})$ (which is independent of the choice of $\left.r^{\prime}\right)$.

For each $v \in R \cup S_{l}$ choose once and for all a place $\widetilde{v}$ of $F$ lying above $v$. Let $\widetilde{R}$ and $\widetilde{S}_{l}$ denote the set of $\widetilde{v}$ for $v$ in $R$ and $S$ respectively. For each $v \in R$, let $R_{\left.\vec{r}\right|_{G_{F}}}$ 
denote the universal $\mathcal{O}$-lifting ring of $\left.\bar{r}\right|_{G_{F_{\tilde{v}}}}$. Suppose that for each $v \in R$ and each minimal prime ideal $\wp$ of $\left.R_{\bar{r}}^{\square}\right|_{G_{F}}$, the quotient $\left.R_{\bar{r}}^{\square}\right|_{G_{F}} / \wp$ is geometrically integral. Note that this can always be achieved by replacing $\mathcal{O}$ with the ring of integers in a finite extension of $K$. Suppose that the lift $\left.r^{\prime}\right|_{G_{F_{\tilde{v}}}}$ corresponds to a closed point of $\operatorname{Spec} R_{\left.\widetilde{r}\right|_{G_{\widetilde{v}}} ^{\square}}[1 / l]$ which lies on only one irreducible component. Let $R_{\widetilde{v}}$ denote the quotient of $\left.R_{\bar{r}}^{\square}\right|_{G_{\widetilde{v}}}$ by the minimal prime ideal corresponding to this irreducible component.

For $v \in S_{l}$, let $\lambda_{\widetilde{v}}$ be the element of $\left(\mathbb{Z}_{+}^{n}\right)^{\operatorname{Hom}\left(F_{\widetilde{v}}, K\right)}$ with the property that $\left.r^{\prime}\right|_{G_{F_{\tilde{v}}}}$ has $l$-adic Hodge type $\mathbf{v}_{\lambda_{\tilde{v}}}$. Suppose that for each minimal prime ideal $\wp$ of $R_{\left.\bar{r}\right|_{G_{F}}}^{\mathbf{v}_{F_{\tilde{v}}}, c r}$, the quotient $R_{\left.\bar{r}\right|_{G_{\tilde{v}}}}^{\mathbf{v}_{\lambda_{\widetilde{v}}}, c r} / \wp$ is geometrically integral. Let $R_{\widetilde{v}}$ be the quotient of $R_{\left.\bar{r}\right|_{G_{F}}}^{\mathbf{v}_{\lambda_{\tilde{v}}}, c r}$ by the minimal prime ideal corresponding to the (necessarily unique) irreducible component of Spec $R_{\left.\bar{r}\right|_{G_{F}} ^{\mathbf{v}_{\widetilde{v}}}, c r}[1 / l]$ containing $\left.r^{\prime}\right|_{G_{F_{\widetilde{v}}}}$.

Consider the deformation problem

$$
\mathcal{S}_{r^{\prime}}:=\left(F / F^{+}, R \cup S_{l}, \widetilde{R} \cup \widetilde{S}_{l}, \mathcal{O}, \bar{r}, \epsilon^{1-n} \delta_{F / F^{+}}^{\mu},\left\{R_{\widetilde{v}}\right\}_{v \in R \cup S_{l}}\right) .
$$

By Lemma 3.5.1, the rings $R_{\widetilde{v}}$ for $v \in S_{l} \cup R$ satisfy the property $\left(^{*}\right)$ of section 3.5. Let $R_{\mathcal{S}_{r^{\prime}}}^{\text {univ }}$ be the object of $\mathcal{C}_{\mathcal{O}}$ representing the corresponding deformation functor.

Proposition 3.6.3. Maintain the assumptions made above. Suppose in addition that

(i) $\bar{\rho}\left(G_{F\left(\zeta_{l}\right)}\right) \subset \mathrm{GL}_{n}(k)$ is big, and

(ii) $\bar{F}^{\text {ker ad } \bar{\rho}}$ does not contain $\zeta_{l}$.

Then:

(1) $R_{\mathcal{S}_{r^{\prime}}}^{\text {univ }}$ is a finite $\mathcal{O}$-algebra.

(2) Any $\overline{\mathbb{Q}}_{l}$-point of $R_{\mathcal{S}_{r^{\prime}}}^{\text {univ }}$ gives rise to a representation $G_{F} \rightarrow \mathrm{GL}_{n}\left(\overline{\mathbb{Q}}_{l}\right)$ which is automorphic of level prime to $l$.

(3) $\mu \equiv n \bmod 2$.

Proof. Choose a place $v_{1}$ of $F$ not lying over $l$ and such that

- $v_{1}$ is unramified over a rational prime $p$ with $\left[F\left(\zeta_{p}\right): F\right]>n$;

- $v_{1}$ does not split completely in $F\left(\zeta_{l}\right)$;

- $\rho$ and $\pi$ are unramified at $v_{1}$;

- $\operatorname{ad} \bar{\rho}\left(\operatorname{Frob}_{v_{1}}\right)=1$.

Choose an imaginary $\mathrm{CM}$ field $L / F$ such that:

- $L / F$ is solvable.

- $L$ is linearly disjoint from $\bar{F}^{\operatorname{ker} \bar{r}}\left(\zeta_{l}\right)$ over $F$.

- $4 \mid\left[L^{+}: F^{+}\right]$, where $L^{+}$denotes the maximal totally real subfield of $L$.

- $L / L^{+}$is unramified at all finite places.

- Every place of $L$ over $v_{1}$ or $c v_{1}$ is split over $L^{+}$. Moreover, $v_{1}$ and $c v_{1}$ split completely in $L$.

- The places $v \in R \cup S_{l}$ split completely in $L^{+}$.

Let $S_{L, l}$ denote the set of places of $L^{+}$dividing $l$ and let $R_{L}$ denote the set of places of $L^{+}$lying over $R$. Similarly, let $\widetilde{S}_{L, l}$ and $\widetilde{R}_{L}$ denote the sets of places of $L$ lying over $\widetilde{S}_{l}$ and $\widetilde{R}$ respectively. Let $S_{L, a}$ denote the set of places of $L^{+}$lying over the 
restriction of $v_{1}$ to $F^{+}$. Let $\widetilde{S}_{L, a}$ denote the set of places of $L$ lying over $v_{1}$. Let $T=S_{L, l} \sqcup R_{L} \sqcup S_{L, a}$ and let $\widetilde{T}=\widetilde{S}_{L, l} \sqcup \widetilde{R}_{L} \sqcup \widetilde{S}_{L, a}$ so that $\widetilde{T}$ consists of one place $\widetilde{v}$ for each place $v \in T$.

For $v \in R_{L} \cup S_{L, l}$, let $R_{\widetilde{v}}=R_{\left.\widetilde{v}\right|_{F}}$, regarded as a quotient of $R_{\left.\bar{r}\right|_{G_{L}}}$ (note that $F_{\left.\widetilde{v}\right|_{F}} \stackrel{\sim}{\longrightarrow} L_{\widetilde{v}}$ by the choice of $\left.L\right)$. For $v \in R_{L} \cup S_{L, a}$, let $\bar{R}_{\left.\bar{r}\right|_{G_{L}}}$ be the maximal $l$ -

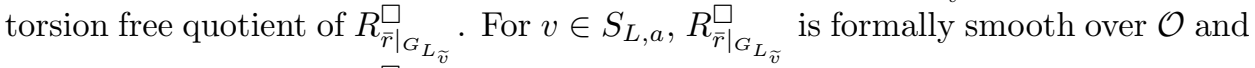

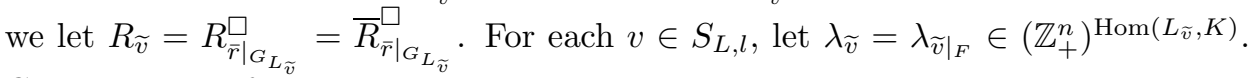
Consider the deformation problems

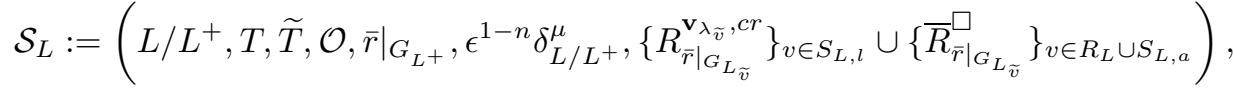

$$
\begin{aligned}
& \mathcal{S}_{L, r^{\prime}}:=\left(L / L^{+}, T, \widetilde{T}, \mathcal{O},\left.\bar{r}\right|_{G_{L^{+}}}, \epsilon^{1-n} \delta_{L / L^{+}}^{\mu},\left\{R_{\widetilde{v}}\right\}_{v \in T}\right) .
\end{aligned}
$$

By Lemma 3.5.1, the rings $R_{\left.\bar{r}\right|_{G_{L}}}^{\mathbf{v}_{\lambda_{\tilde{v}}}, c r}, R_{\widetilde{v}}$ for $v \in S_{L, l}, \bar{R}_{\left.\bar{r}\right|_{G_{L}}}^{\square}, R_{\widetilde{v}}$ for $v \in R_{L}$ and $\bar{R}_{\left.\bar{r}\right|_{G_{L}}}^{\square}$ for $v \in S_{L, a}$ satisfy the property $(*)$ of Section 3.5 . Let $R_{\mathcal{S}_{L}}^{\text {univ }}$ and $R_{\mathcal{S}_{L, r^{\prime}}}^{\text {univ }}$ be the objects of $\mathcal{C}_{\mathcal{O}}$ representing the corresponding deformation functors. Note that there is a natural surjection $R_{\mathcal{S}_{L}}^{\text {univ }} \rightarrow R_{\mathcal{S}_{L, r^{\prime}}}^{\text {univ }}$. Note also that there is a natural

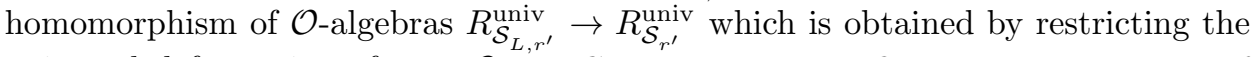
universal deformation of type $\mathcal{S}_{r^{\prime}}$ to $G_{L^{+}}$. This map is finite by an argument of Khare and Wintenberger (cf. Lemma 3.2.5 of [GG09]), and hence it suffices to show that $R_{\mathcal{S}_{L, r^{\prime}}}^{\text {univ }}$ is finite over $\mathcal{O}$.

Let $G_{/ \mathcal{O}_{L^{+}}}$be an algebraic group as in section 3.2 (with $F / F^{+}$replaced by $L / L^{+}$). By Théorème 5.4 and Corollaire 5.3 of [Lab09] there exists an automorphic representation $\Pi$ of $G\left(\mathbb{A}_{L^{+}}\right)$such that $\pi_{L}$ is a strong base change of $\Pi$. Let $U=$ $\prod_{v} U_{v} \subset G\left(\mathbb{A}_{L^{+}}^{\infty}\right)$ be a compact open subgroup such that

- $U_{v}=G\left(\mathcal{O}_{L_{v}^{+}}\right)$for $v \in S_{L, l}$ and for $v \notin T$ split in $L$;

- $U_{v}$ is a hyperspecial maximal compact subgroup of $G\left(L_{v}^{+}\right)$for each $v$ inert in $L$;

- $U_{v}$ is such that $\Pi_{v}^{U_{v}} \neq\{0\}$ for $v \in R_{L}$;

- $U_{v}=\operatorname{ker}\left(G\left(\mathcal{O}_{L_{v}^{+}}\right) \rightarrow G\left(k_{v}\right)\right)$ for $v \in S_{L, a}$.

Let $\widetilde{I}_{l}$ denote the set of embeddings $L \hookrightarrow K$ giving rise to one of the places $\widetilde{v} \in \widetilde{S}_{L, l}$. Let $\lambda=\left(\lambda_{\widetilde{v}}\right)_{v \in S_{L, l}}$ regarded as an element of $\left(\mathbb{Z}_{+}^{n}\right)^{\widetilde{I}_{l}}$ in the evident way and let $S_{\lambda}(U, \mathcal{O})$ be the space of $l$-adic automorphic forms on $G$ of weight $\lambda$ introduced above. Let $\mathbb{T}_{\lambda}^{T}(U, \mathcal{O})$ be the $\mathcal{O}$-subalgebra of $\operatorname{End}_{\mathcal{O}}\left(S_{\lambda}(U, \mathcal{O})\right)$ generated by the Hecke operators $T_{w}^{(j)},\left(T_{w}^{(n)}\right)^{-1}$ for $w$ a place of $L$ split over $L^{+}$, not lying over $T$ and $j=1, \ldots, n$. The eigenvalues of the operators $T_{w}^{(j)}$ on the space $\left(\iota^{-1} \Pi^{\infty}\right)^{U}$ give rise to a homomorphism of $\mathcal{O}$-algebras $\mathbb{T}_{\lambda}^{T}(U, \mathcal{O}) \rightarrow \overline{\mathbb{Q}}_{l}$. Extending $K$ if necessary, we can and do assume that this homomorphism takes values in $\mathcal{O}$. Let $\mathfrak{m}$ denote the unique maximal ideal of $\mathbb{T}_{\lambda}^{T}(U, \mathcal{O})$ containing the kernel of this homomorphism.

Note that $\left.\bar{r}\right|_{G_{L}}$ is $\mathrm{GL}_{n}(k)$-conjugate to $\bar{r}_{\mathfrak{m}}$, where $\bar{r}_{\mathfrak{m}}$ is the representation associated to the maximal ideal $\mathfrak{m}$ of $\mathbb{T}_{\lambda}^{T}(U, \mathcal{O})$ in Section 3.2. After conjugating we can and do assume that $\left.\bar{r}\right|_{G_{L}}=\bar{r}_{\mathfrak{m}}$. Since $\mathfrak{m}$ is non-Eisenstein we have a continuous lift

$$
r_{\mathfrak{m}}: G_{L^{+}} \rightarrow \mathcal{G}_{n}\left(\mathbb{T}_{\lambda}^{T}(U, \mathcal{O})_{\mathfrak{m}}\right)
$$


of $\bar{r}$. Properties $(0)-(3)$ of $r_{\mathfrak{m}}$ and the fact that $\mathbb{T}_{\lambda}^{T}(U, \mathcal{O})_{\mathfrak{m}}$ is $l$-torsion free and reduced imply that $r_{\mathfrak{m}}$ is of type $\mathcal{S}_{L}$. Hence $r_{\mathfrak{m}}$ gives rise to an $\mathcal{O}$-algebra homomorphism

$$
R_{\mathcal{S}_{L}}^{\text {univ }} \rightarrow \mathbb{T}_{\lambda}^{T}(U, \mathcal{O})_{\mathfrak{m}}
$$

(which is surjective by property (2) of $r_{\mathfrak{m}}$ ).

We define

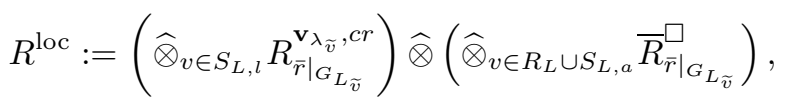

where all completed tensor products are taken over $\mathcal{O}$. Note that $R^{\text {loc }}$ is equidimensional of dimension $1+n^{2} \# T+\left[L^{+}: \mathbb{Q}\right] n(n-1) / 2$ by Lemma 3.3 of [BLGHT09].

Sublemma. There are integers $q, g \in \mathbb{Z}_{\geq 0}$ with

$$
1+q+n^{2} \# T=\operatorname{dim} R^{\text {loc }}+g
$$

and a module $M_{\infty}$ for both $R_{\infty}:=R^{\operatorname{loc}}\left[\left[x_{1}, \ldots, x_{g}\right]\right]$ and $S_{\infty}:=\mathcal{O}\left[\left[z_{1}, \ldots, z_{n^{2} \# T}\right.\right.$, $\left.\left.y_{1}, \ldots, y_{q}\right]\right]$ such that:

(1) $M_{\infty}$ is finite and free over $S_{\infty}$.

(2) $M_{\infty} /\left(z_{i}, y_{j}\right) \cong S_{\lambda}(U, \mathcal{O})_{\mathfrak{m}}$.

(3) The action of $S_{\infty}$ on $M_{\infty}$ can be factored through an $\mathcal{O}$-algebra homomorphism $S_{\infty} \rightarrow R_{\infty}$.

(4) There is a surjection $R_{\infty} \rightarrow R_{\mathcal{S}_{L}}^{\text {univ }}$ whose kernel contains all the $z_{i}$ and $y_{j}$ and which is compatible with the actions of $R_{\infty} /\left(z_{i}, y_{j}\right)$ and $R_{\mathcal{S}_{L}}^{\mathrm{univ}}$ on $M_{\infty} /$ $\left(z_{i}, y_{j}\right) \cong S_{\lambda}(U, \mathcal{O})_{\mathfrak{m}}$. Moreover, there is a lift $r_{\mathcal{S}_{L}}^{\text {univ }}: G_{L^{+}} \rightarrow \mathcal{G}_{n}\left(R_{\mathcal{S}_{L}}^{\text {univ }}\right)$ of $\bar{r}$ representing the universal deformation so that for each $v \in T$, the composite $R_{\left.\bar{r}\right|_{G_{\tilde{v}}}}^{\square} \rightarrow R^{\text {loc }} \rightarrow R_{\infty} \rightarrow R_{\mathcal{S}_{L}}^{\text {univ }}$ arises from the lift $\left.r_{\mathcal{S}_{L}}^{\text {univ }}\right|_{G_{L_{\tilde{v}}}}$.

Moreover, $\mu \equiv n \bmod 2$.

This is proved in exactly the same way as the sublemma in the proof of Theorem 3.6.1 (for the fact that $\mu \equiv n \bmod 2$, see the penultimate sentence of loc. cit.). Points (1) and (3) tell us that the support of $M_{\infty}$ in Spec $R_{\infty}$ is a union of irreducible components. Let

$$
R_{r^{\prime}}^{\text {loc }}=\widehat{\bigotimes_{v \in T}} R_{\widetilde{v}}
$$

Then $R_{r^{\prime}}^{\text {loc }}$ is a quotient of $R^{\text {loc }}$ corresponding to an irreducible component (see part 5 of Lemma 3.3 of [BLGHT09]). Let $R_{\infty, r^{\prime}}=R_{\infty} \otimes_{R^{\text {loc }}} R_{r^{\prime}}^{\text {loc }}$. Again $R_{\infty, r^{\prime}}$ is a quotient of $R_{\infty}$ corresponding to an irreducible component. The lift $\left.r^{\prime}\right|_{G_{L^{+}}}$ of $\left.\bar{r}\right|_{G_{L}+}$ gives rise to a closed point of $\operatorname{Spec} R_{\infty}[1 / l]$ which lies in the support of $M_{\infty}$ and which lies in Spec $R_{\infty, r^{\prime}}[1 / l]$ but in no other irreducible component of $\operatorname{Spec} R_{\infty}[1 / l]$. We deduce that $\operatorname{Spec} R_{\infty, r^{\prime}}$ is contained in the support of $M_{\infty}$. In other words, in the terminology of section 2 of [Tay08], $M_{\infty} \otimes_{R_{\infty}} R_{\infty, r^{\prime}}$ is a nearly faithful $R_{\infty, r^{\prime}}$-module. It follows (by Lemma 2.2 of [Tay08) that $M_{\infty} \otimes_{R_{\infty}} R_{\mathcal{S}_{L, r^{\prime}}}^{\text {univ }}$ is a nearly faithful $R_{\mathcal{S}_{L, r^{\prime}}}^{\text {univ }}$-module. Note that $M_{\infty} \otimes_{R_{\infty}} R_{\mathcal{S}_{L, r^{\prime}}}^{\text {univ }}$ is a finite $\mathcal{O}$-module, being a quotient of $S_{\lambda}(U, \mathcal{O})_{\mathfrak{m}}$. Let $I$ denote the annihilator of $M_{\infty} \otimes_{R_{\infty}} R_{\mathcal{S}_{L, r^{\prime}}}^{\text {univ }}$ in $R_{\mathcal{S}_{L, r^{\prime}}}^{\text {univ }}$. Then $R_{\mathcal{S}_{L, r^{\prime}}}^{\text {univ }} / I$ is finite over $\mathcal{O}$. The same is true of $\left(R_{\mathcal{S}_{L, r^{\prime}}}^{\text {univ }}\right)^{\text {red }}$ since $I$ is nilpotent. It follows that $R_{\mathcal{S}_{L, r^{\prime}}}^{\mathrm{univ}} / \mathfrak{m}_{\mathcal{O}}$ is Artinian (being Noetherian of dimension 0) and hence $R_{\mathcal{S}_{L, r^{\prime}}}^{\text {univ }}$ is finite over $\mathcal{O}$ by the topological version of Nakayama's lemma. 
We have established parts (1) and (3) of the proposition. For part (2), it is clear from the above that any $\overline{\mathbb{Q}}_{l}$-point of $R_{\mathcal{S}}^{\text {univ }}$ gives rise to a representation $G_{F} \rightarrow \mathrm{GL}_{n}\left(\overline{\mathbb{Q}}_{l}\right)$ which becomes automorphic upon restriction to $G_{L}$ and hence is automorphic by Lemma 1.4 of [BLGHT09] (since $L / F$ is solvable).

\section{A CHARACTER-BUILDING EXERCISE}

4.1. The main purpose of this section is to prove a lemma allowing us to construct Galois characters with certain properties. Before we do so, we must discuss the notion of bigness and prove a result which will allow us to deduce that representations are 'big' in certain circumstances.

We begin by recalling the definition of $m$-big from [BLGHT09].

Definition 4.1.1. Let $k / \mathbb{F}_{l}$ be algebraic and $m$ a positive integer. We say that a subgroup $H \subset \mathrm{GL}_{n}(k)$ of $\mathrm{GL}_{n}(k)$ is $m$-big if the following conditions are satisfied:

- $H$ has no $l$-power order quotient.

- $H^{0}\left(H, \mathfrak{s l}_{n}(k)\right)=(0)$.

- $H^{1}\left(H, \mathfrak{s l}_{n}(k)\right)=(0)$.

- For all irreducible $k[H]$-submodules $W$ of $\mathfrak{g l}_{n}(k)$ we can find $h \in H$ and $\alpha \in k$ such that:

$-\alpha$ is a simple root of the characteristic polynomial of $h$, and if $\beta$ is any other root, then $\alpha^{m} \neq \beta^{m}$.

- Let $\pi_{h, \alpha}$ (respectively $i_{h, \alpha}$ ) denote the $h$-equivariant projection from $k^{n}$ to the $\alpha$-eigenspace of $h$ (respectively the $h$-equivariant injection from the $\alpha$-eigenspace of $h$ to $\left.k^{n}\right)$. Then $\pi_{h, \alpha} \circ W \circ i_{h, \alpha} \neq 0$.

We simply write "big" for 1-big. If $\bar{r}$ is a representation of some group valued in $\mathrm{GL}_{n}(k)$, then we say that $\bar{r}$ has $m$-big image if the image of $\bar{r}$ is $m$-big. If $K$ is an algebraic extension of $\mathbb{Q}_{l}$ with residue field $k$ and $r$ is a representation of some group valued in $\mathrm{GL}_{n}(K)$, then we say that $r$ has $m$-big image if $\bar{r}$ has $m$-big image, where $\bar{r}$ is the semisimplification of the reduction $\bmod l$ of $r$.

The following lemma is essentially implicit in the proof of Theorem 7.6 of BLGHT09, but it is hard to extract by reference the material we need from the proof there, so we will give a self-contained statement and proof.

Lemma 4.1.2. Suppose that $F$ is a totally real field, $l$ is a rational prime, $m^{*} i s$ a positive even integer not divisible by $l, n$ is a positive integer with $l>2 n-2$, $r: G_{F} \rightarrow \mathrm{GL}_{n}\left(\overline{\mathbb{Z}}_{l}\right)$ is a continuous l-adic Galois representation, and $M$ is a cyclic $C M$ extension of $F$ of degree $m^{*}$ such that:

- $M$ is linearly disjoint from $\bar{F}^{\mathrm{ker}} \bar{r}\left(\zeta_{l}\right)$ over $F$, and

- every prime $v$ of $F$ above $l$ is unramified in $M$.

Suppose also that $\theta^{\prime}: G_{M} \rightarrow \overline{\mathbb{Z}}_{l}^{\times}$is a continuous character.

(1) Suppose $\left[\bar{F}^{\text {kerad } \bar{r}}\left(\zeta_{l}\right): \bar{F}^{\text {kerad } \bar{r}}\right]>m^{*}$. Then the fixed field of the kernel of the representation $\operatorname{ad}\left(\bar{r} \otimes \operatorname{Ind}_{G_{M}}^{G_{F}} \bar{\theta}^{\prime}\right)$ does not contain $\zeta_{l}$.

(2) Suppose that $\left.r\right|_{G_{F\left(\zeta_{l}\right)}}$ has $m^{*}$-big image and that $\left(\bar{\theta}^{\prime}\right)\left(\bar{\theta}^{\prime}\right)^{c}$ can be extended to $G_{F}$. Suppose further that there is a prime $\mathfrak{Q}$ of $M$ lying above a prime $\mathfrak{q}$ of $F$ lying in turn above a rational prime $q$, such that:

- $r$ is unramified at all primes above $q$,

- $q \neq l$, 
- $q$ splits completely in $M$,

- $q$ is unramified in $\bar{F}^{\text {ker }} \bar{r}\left(\zeta_{l}\right)$,

- $q-1>2 n$,

- $\left(\theta^{\prime}\right)\left(\theta^{\prime}\right)^{c}$ is unramified at primes above $q$, and

- $q \mid \# \theta^{\prime}\left(I_{\mathfrak{Q}}\right)$ (and so $q \mid \# \theta^{\prime}\left(I_{\mathfrak{Q}^{c}}\right)$ ), and $\theta^{\prime}$ is unramified at all primes above $\mathfrak{q}$ except $\mathfrak{Q}$ and $\mathfrak{Q}^{c}$.

Then $\left.\left(r \otimes \operatorname{Ind}_{G_{M}}^{G_{F}} \theta^{\prime}\right)\right|_{G_{F\left(\zeta_{l}\right)}}$ has big image.

Proof. We will begin by proving the first part; so suppose that $r$ has the property assumed there. By the given assumption, there is an element of $\operatorname{Gal}\left(\bar{F}^{\operatorname{ker} a d} \bar{r}\left(\zeta_{l}\right) /\right.$ $\bar{F}^{\text {ker ad } \bar{r}}$ ) of order larger than $m^{*}$; using the assumption that $M$ and $\bar{F}^{\text {ker } \bar{r}}\left(\zeta_{l}\right) \supset$ $\bar{F}^{\text {ker ad } \bar{r}}\left(\zeta_{l}\right)$ are linearly disjoint over $F$, we can consider this as an element of $\operatorname{Gal}\left(M \bar{F}^{\operatorname{kerad} \bar{r}}\left(\zeta_{l}\right) / \bar{F}^{\text {kerad } \bar{r}} M\right)$ with the same property, and lift this to an element $\sigma$ of $\operatorname{Gal}\left(\bar{F} / M \bar{F}^{\operatorname{ker} a d} \bar{r}\right)$. (This element will have the property that $\bar{\epsilon}_{l}(\sigma)$ has order $>m^{*}$.) Let $\tau$ be a generator of $\operatorname{Gal}(M / F)$. Then lift $\tau$ to an element $\tilde{\tau}$ of $G_{F}$; let

$$
\sigma^{\prime}=\prod_{i=0}^{m^{*}-1} \tilde{\tau}^{-i} \sigma \tilde{\tau}^{i}
$$

and notice that $\operatorname{ad}\left(\bar{r} \otimes \operatorname{Ind}_{G_{M}}^{G_{F}} \bar{\theta}^{\prime}\right)$ is trivial on $\sigma^{\prime}$, while $\bar{\epsilon}_{l}\left(\sigma^{\prime}\right)=\bar{\epsilon}_{l}(\sigma)^{m^{*}} \neq 1$. This proves (1).

We now turn to proving the second part; so let us assume that we have $k, q, \mathfrak{q}, \mathfrak{Q}$ with the properties stipulated there. We will follow the proof of Theorem 7.6 of [BLGHT09] very closely. Since $l>2 n-2$, the main result of [Ser94] shows that $\left.\operatorname{ad} \bar{r}\right|_{G_{F\left(\zeta_{l}\right)}}$ is semisimple, and we may write

$$
\left.\operatorname{ad} \bar{r}\right|_{G_{F\left(\zeta_{l}\right)}}=V_{0}^{\oplus m_{0}} \oplus V_{1}^{\oplus m_{1}} \oplus \cdots \oplus V_{s}^{\oplus m_{s}},
$$

where the $V_{i}$ are pairwise non-isomorphic, irreducible $\overline{\mathbb{F}}_{l}\left[G_{F\left(\zeta_{l}\right)}\right]$-modules and the $m_{i}$ are positive integers. Let $V_{0}=1$. By the assumption that $\left.r\right|_{G_{F\left(\zeta_{l}\right)}}$ has $m^{*}$ big image, $m_{0}=1$. Adopting $r^{\prime}$ as an abbreviated name for $\operatorname{Ind}_{G_{M}}^{G_{F}} \theta^{\prime}$, let us choose $e_{0}, \ldots, e_{m^{*}-1}$ to be a basis for $r^{\prime}$ as follows. First, choose $\tilde{\tau} \in G_{F}$ a lifting of $\tau \in \operatorname{Gal}(M / F)$. Then, choose a non-zero primitive vector $e_{0}$ in $r^{\prime}$ such that $r^{\prime}(\sigma) e_{0}=\theta^{\prime}(\sigma) e_{0}$ for all $\sigma \in G_{M}$, and set $e_{i}=r^{\prime}\left(\tilde{\tau}^{i}\right) e_{0}$ for $i=1, \ldots, m^{*}-1$. Note that this means that $G_{M}$ acts on $e_{i}$ via the character $\theta^{\prime \tilde{\tau}^{-i}}$. Moreover,

$$
r^{\prime}(\tilde{\tau})\left(e_{m^{*}-1}\right)=r^{\prime}\left(\tilde{\tau}^{m^{*}}\right) e_{0}=\theta^{\prime}\left(\tilde{\tau}^{m^{*}}\right) e_{0} .
$$

Now let $f_{0}, \ldots, f_{m^{*}-1}$ be the basis of $\operatorname{Hom}\left(r^{\prime}, \overline{\mathbb{Z}}_{l}\right)$ dual to $e_{0}, \ldots, e_{m^{*}-1}$. Let us quickly establish a sublemma:

Sublemma. Suppose there is some character $\psi: G_{M} \rightarrow \overline{\mathbb{F}}_{l}^{\times}$which is unramified at primes above $q$ such that $\bar{\theta}^{\prime} / \bar{\theta}^{\tau^{k}}=\bar{\theta}^{\prime} \tilde{\tau}^{l} / \bar{\theta}^{\prime} \tilde{\tau}^{m} \psi$ (or equivalently $\bar{\theta}^{\prime} \bar{\theta}^{\prime} \tilde{\tau}^{m}=\bar{\theta}^{\prime} \tilde{\tau}^{l} \bar{\theta}^{\prime} \tilde{\tau}^{k} \psi$ ); then either $k=m$ and $l=0, k=0$ and $l=m$, or $l=\left(m^{*} / 2\right)+k$ and $m=m^{*} / 2$. Conversely, if $k=m$ and $l=0$, then $\overline{\theta^{\prime}} / \bar{\theta}^{\tilde{r}^{k}}=\bar{\theta}^{\prime} \tilde{\tau}^{l} / \bar{\theta}^{\prime} \tilde{\tau}^{m}$, and the same is true if $k=0$ and $l=m$, or if $l=\left(m^{*} / 2\right)+k$ and $m=m^{*} / 2$.

Proof. For the first part, we consider the action of inertia above $\mathfrak{q}$ on each side of $\overline{\theta^{\prime}} \bar{\theta}^{\prime} \tilde{\tau}^{m}=\bar{\theta}^{\prime} \tilde{\tau}^{l} \bar{\theta}^{\prime} \tilde{\tau}^{k}$. 
We first set up some notation. Let $\iota_{I_{\mathfrak{Q}}}$ denote the inclusion of $I_{\mathfrak{Q}}$ into $G_{M}$, and let $\delta:=\bar{\theta}^{\prime} \circ \iota_{I_{\mathfrak{Q}}}$. (Note that $\delta$ is not trivial, by our assumption that $q \mid \# \theta^{\prime}\left(I_{\mathfrak{Q}}\right)$.) For $i$ an integer, let $c_{\tilde{\tau}^{i}}: G_{M} \rightarrow G_{M}$ denote the 'conjugation by $\tilde{\tau}^{i}$ ' map, and let $\delta_{i}:=\bar{\theta}^{\prime} \circ c_{\tilde{\tau}^{i}} \circ \iota_{I_{\mathfrak{Q}}}$. Observe that $\delta_{0}=\delta$, that $\delta_{i}$ is trivial for $i \neq 0, m^{*} / 2$ (by the assumption that $\theta^{\prime}$ is unramified at all primes above $\mathfrak{q}$ except $\mathfrak{Q}$ and $\mathfrak{Q}^{c}$ ), and that $\delta_{m^{*} / 2}=\delta^{-1}\left(\right.$ since $\left.\delta_{m^{*} / 2}=\left(\bar{\theta}^{\prime} \circ c_{\tilde{\tau}^{m^{*} / 2}} \circ \iota_{I_{\mathfrak{Q}}}\right)=\left({\overline{\theta^{\prime}}}^{\tilde{\tau}^{m^{*} / 2}} \circ \iota_{I_{\mathfrak{Q}}}\right)=\left({\overline{\theta^{\prime}}}^{c}\right) \circ \iota_{I_{\mathfrak{Q}}}\right)=$ $\left.\left(1 / \bar{\theta}^{\prime}\right) \circ \iota_{I_{\mathfrak{Q}}}\right)=1 / \delta$, where the penultimate equality is by our assumption that $\left(\theta^{\prime}\right)\left(\theta^{\prime}\right)^{c}$ is unramified at primes above $\left.q\right)$.

Now let us consider the maps $\beta_{i}:=\left(\bar{\theta}^{\prime} \bar{\theta}^{, \tilde{\tau}^{m}}\right) \circ c_{\tilde{\tau}^{i}} \circ \iota_{I_{\mathfrak{Q}}}$ for each $i$. These maps are clearly trivial unless $i=0, m^{*} / 2, m$ or $m+\left(m^{*} / 2\right)$ (considering $\left.i \bmod m^{*}\right)$. Let us split into cases:

- We have $m \neq\left(m^{*} / 2\right), 0$. In this case, it is easy to see that the values $i=0$, $m^{*} / 2, m$ or $m+\left(m^{*} / 2\right) \bmod m^{*}$ are distinct. It is also easy to see that $\beta_{i}$ for these $i$ values are (respectively) $\delta, \delta^{-1}, \delta, \delta^{-1}$, where $\delta$ is some character.

On the other hand, consider $\beta_{i}^{\prime}:=\left(\bar{\theta}^{\tau^{l}} \bar{\theta}^{\tilde{\tau}^{k}} \psi\right) \circ c_{\tilde{\tau}^{i}} \circ \iota_{I_{\mathfrak{Q}}}$. For these maps to be non-trivial for 4 distinct values of $i \bmod m^{*}$, as they must, we need $l \neq k, k+\left(m^{*} / 2\right)$; then it will be non-trivial for $i=l, l+\left(m^{*} / 2\right)$, $k, k+\left(m^{*} / 2\right)$, for which values of $i$ we get $\delta, \delta^{-1}, \delta, \delta^{-1}$ respectively; and comparing, we conclude that $l=0$ and $k=m$ or $l=m$ and $k=0$ (since $\left.\delta \neq \delta^{-1}\right)$.

- We have $m=0$. In this case, $\beta_{i}$ is non-trivial for $i=0$ or $i=m^{*} / 2(\bmod$ $m^{*}$ ), which are distinct, and the corresponding $\beta_{i}$ are (respectively) $\delta^{2}, \delta^{-2}$. Now consider $\beta_{i}^{\prime}:=\left(\bar{\theta}^{\prime} \tilde{\tau}^{l} \bar{\theta}^{\tau^{k}} \psi\right) \circ c_{\tilde{\tau}^{i}} \circ \iota_{I_{\mathfrak{Q}}}$. For these maps to be non-trivial for at most two values of $i$ we need $l=k$ or $l=k+\left(m^{*} / 2\right)$, and in the latter case we see that all $\beta_{i}^{\prime}$ are in fact trivial. Thus we must have $l=k$. Comparing the $i$ for which $\beta_{i}$ and $\beta_{i}^{\prime}$ are non-trivial, we see that $l=0$.

- We have $m=m^{*} / 2$. In this case, it is easy to see that all $\beta_{i}$ are trivial. Now, considering $\beta_{i}^{\prime}:=\left(\bar{\theta}^{, \tilde{\tau}^{l}} \bar{\theta}^{, \tilde{\tau}^{k}} \psi\right) \circ c_{\tilde{\tau}^{i}} \circ \iota_{I_{\mathfrak{Q}}}$, we see that for these maps to be non-trivial for at most two values of $i$ (as they must be) we need $l=k$ or $l=k+\left(m^{*} / 2\right)$, and we see that only in the latter case is it in fact trivial for all values of $i$.

For the converse, if $k=m$ and $l=0$, the fact that $\bar{\theta}^{\prime} / \bar{\theta}^{\prime} \tilde{\tau}^{k}=\bar{\theta}^{\prime} / \bar{\theta}^{\tau^{k}}$ is trivial. Similarly, if $k=0$ and $l=m$, we need to prove that $\bar{\theta}^{\prime} / \bar{\theta}^{\prime}=\bar{\theta}^{\prime} \tilde{\tau}^{m} / \bar{\theta}^{\prime} \tilde{\tau}^{m}$, which is also trivial. Finally, if $l=\left(m^{*} / 2\right)+k$ and $m=m^{*} / 2$, it remains to show that $\left(\bar{\theta}^{\prime}\right)\left(\bar{\theta}^{\prime}\right)^{c}=\left(\left(\bar{\theta}^{\prime}\right)\left(\bar{\theta}^{\prime}\right)^{c}\right)^{k}$ (where we write $c$ for complex conjugation), which is true since one of our hypotheses is that $\left(\bar{\theta}^{\prime}\right)\left(\bar{\theta}^{\prime}\right)^{c}$ can be extended to $G_{F}$.

We can now decompose:

$$
\left.\operatorname{ad} \bar{r}^{\prime}\right|_{G_{F\left(\zeta_{l}\right)}}=\left(\bigoplus_{\chi \in \operatorname{Hom}\left(\operatorname{Gal}\left(M\left(\zeta_{l}\right) / F\left(\zeta_{l}\right)\right), \overline{\mathbb{F}}_{l}^{\times}\right)} W_{\chi}\right) \oplus\left(\bigoplus_{i=1}^{m^{*}-1} W_{i}\right),
$$

where:

- $W_{\chi}$ is the span of $\sum_{i=0}^{m^{*}-1} \chi^{-1}\left(\tau^{i}\right) e_{i} \otimes f_{i}$, so $W_{\chi} \cong \overline{\mathbb{F}}_{l}(\chi)$.

- $W_{i}$ is the span of $\left\{e_{j} \otimes f_{i+j}\right\}_{j=0, \ldots, m^{*-1}}$, so $W_{i} \cong \operatorname{Ind}_{G_{M\left(\zeta_{l}\right)}}^{G_{F\left(\zeta_{l}\right)}}\left(\bar{\theta}^{\prime} / \bar{\theta}^{\prime} \tilde{\tau}^{-i}\right)$. 
From this we turn to study $\bar{r} \otimes \bar{r}^{\prime}$, which we will abbreviate $\bar{r}^{\prime \prime}$. We can decompose:

$$
\left.\operatorname{ad} \bar{r}^{\prime \prime}\right|_{G_{F\left(\zeta_{l}\right)}}=\left(\bigoplus_{j=0}^{s} \bigoplus_{\chi \in \operatorname{Hom}\left(\operatorname{Gal}\left(M\left(\zeta_{l}\right) / F\left(\zeta_{l}\right)\right), \overline{\mathbb{F}}_{l}^{\times}\right)} V_{j}(\chi)^{m_{j}}\right) \oplus\left(\bigoplus_{j=0}^{s} \bigoplus_{i=1}^{m^{*}-1}\left(V_{j} \otimes W_{i}\right)^{m_{j}}\right) .
$$

We then have the following straightforward lemma:

Lemma. The decomposition (4.1.3) enjoys the following properties:

(1) Each $V_{j}(\chi)$ is irreducible.

(2) We have $V_{j}(\chi) \not \equiv V_{j^{\prime}}\left(\chi^{\prime}\right)$ unless $\chi=\chi^{\prime}$ and $j=j^{\prime}$.

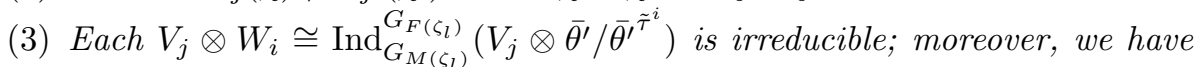
$\operatorname{Ind}_{G_{M\left(\zeta_{l}\right)}}^{G_{F\left(\zeta_{l}\right)}}\left(V_{j} \otimes \bar{\theta}^{\prime} / \bar{\theta}^{\prime} \tilde{\tau}^{i}\right) \nRightarrow \operatorname{Ind}_{G_{M\left(\zeta_{l}\right)}}^{G_{F\left(\zeta_{l}\right)}}\left(V_{j^{\prime}} \otimes \bar{\theta}^{\prime} / \bar{\theta}^{\tilde{\tau}^{i^{\prime}}}\right)$ unless $j=j^{\prime}$ and $i \in$ $\left\{i^{\prime}, m^{*}-i^{\prime}\right\}$.

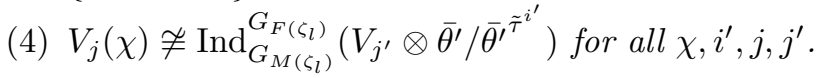

Proof. For part 1, see equation (4.1.1) and the remarks immediately following it. Part 2 is clear, because $M$ is linearly disjoint from $\bar{F}^{\text {ker }} \bar{r}\left(\zeta_{l}\right)$ over $F$. Both of the assertions in part 3 follow from (a) the fact that the only cases where $G_{M\left(\zeta_{l}\right)}$ acts via the same character on $e_{0} \otimes f_{k}$ and $e_{l} \otimes f_{m}$ are when $k=m$ and $l=0, k=0$ and $l=m$, or $l=\left(m^{*} / 2\right)+k$ and $m=m^{*} / 2$ (this fact is our sublemma) and (b) from examining the action of inertia above $q$. Finally, part 4 again follows from a consideration of the action of inertia above $q$.

It is immediate (since there are no terms in the decomposition of ad $\left.\bar{r}^{\prime \prime}\right|_{G_{F\left(\zeta_{l}\right)}}$ isomorphic to 1 other than $\left.V_{0}(1)^{m_{0}}\right)$ that

$$
H^{0}\left(\left(\operatorname{ad} \bar{r}^{\prime \prime}\right)\left(G_{F\left(\zeta_{l}\right)}\right), \operatorname{ad}^{0} \bar{r}^{\prime \prime}\right)=(0) .
$$

Let $H$ (resp. $H^{\prime}$, resp. $\left.H^{\prime \prime}\right)$ denote the image $(\operatorname{ad} \bar{r})\left(G_{F\left(\zeta_{l}\right)}\right)\left(\operatorname{resp} .\left(\operatorname{ad} \bar{r}^{\prime}\right)\left(G_{F\left(\zeta_{l}\right)}\right)\right.$, resp. $\left.\left(\operatorname{ad} \bar{r}^{\prime \prime}\right)\left(G_{F\left(\zeta_{l}\right)}\right)\right)$. We note that the only element of $H$ which is a scalar when thought of as an element of Aut ad $\bar{r}$ is the identity 1$]$ and the same is true for $H^{\prime}$, so that

$$
\begin{array}{r}
H \times H^{\prime} \hookrightarrow \operatorname{Aut}\left(\operatorname{ad} \bar{r}^{\prime \prime}\right) \text { and ker ad }\left.\bar{r}^{\prime \prime}\right|_{G_{F\left(\zeta_{l}\right)}}=\left.\left.\operatorname{ker} \operatorname{ad} \bar{r}\right|_{G_{F\left(\zeta_{l}\right)}} \cap \operatorname{ker} \operatorname{ad} \bar{r}^{\prime}\right|_{G_{F\left(\zeta_{l}\right)}} \\
\left(=K_{\cap}, \text { say }\right) .
\end{array}
$$

Thus, if we define $K_{\cup}=\left\langle\left(\left.\operatorname{ker} \operatorname{ad} \bar{r}\right|_{\left.G_{F\left(\zeta_{l}\right)}\right)},\left(\left.\operatorname{ker} \operatorname{ad} \bar{r}^{\prime}\right|_{G_{F\left(\zeta_{l}\right)}}\right)\right\rangle \triangleleft G_{F\left(\zeta_{l}\right)}\right.$, and $\bar{H}=$ $G_{F\left(\zeta_{l}\right)} / K_{\cup}$, we have maps

$\pi: H=\frac{G_{F\left(\zeta_{l}\right)}}{\left.\operatorname{ker} \operatorname{ad} \bar{r}\right|_{G_{F\left(\zeta_{l}\right)}}} \rightarrow \frac{G_{F\left(\zeta_{l}\right)}}{K_{\cup}}=\bar{H}$ and $\pi^{\prime}: H^{\prime}=\frac{G_{F\left(\zeta_{l}\right)}}{\left.\operatorname{ker} \operatorname{ad} \bar{r}^{\prime}\right|_{G_{F\left(\zeta_{l}\right)}}} \rightarrow \frac{G_{F\left(\zeta_{l}\right)}}{K_{\cup}}=\bar{H}$

\footnotetext{
${ }^{1}$ In case it will help avoid confusion, let us spell this out a little more. The representation $\bar{r}$ can equivalently be thought of as a vector space $V_{\bar{r}}$ on which the Galois group acts; similarly, we can think of the representation ad $\bar{r}$ as a vector space $V_{\mathrm{ad}} \bar{r}$ with an action of the Galois group; the underlying vector space of $V_{\mathrm{ad}} \bar{r}$ is just the vector space End VectSpc $V_{\bar{r}}$ of endomorphisms of the underlying vector space of $V_{\bar{r}}$. Each element $h$ of $H$ determines an element of Aut VectSpc $V_{\text {ad }}$; this is what we mean by $h$ 'thought of as an element of Aut ad $\bar{r}$ '.
} 
such that $G_{F\left(\zeta_{l}\right)} \rightarrow\left\{\left(h, h^{\prime}\right) \in H \times H^{\prime} \mid \pi(h)=\pi^{\prime}\left(h^{\prime}\right)\right\}$. (This is surjective; it suffices to show a) that for each $h \in H$, the image contains $(h, x)$ for some $x$, and b) that the image contains $\left(e, h^{\prime}\right)$ for each $h^{\prime}$ in the kernel of $\pi^{\prime}$, both of which are clear.)

Finally,

$$
\begin{aligned}
\operatorname{ker}\left(G_{F\left(\zeta_{l}\right)}\right. & \left.\rightarrow\left\{\left(h, h^{\prime}\right) \in H \times H^{\prime} \mid \pi(h)=\pi^{\prime}\left(h^{\prime}\right)\right\}\right)=\operatorname{ker}\left(G_{F\left(\zeta_{l}\right)} \rightarrow H \times H^{\prime}\right) \\
& =\operatorname{ker}\left(G_{F\left(\zeta_{l}\right)} \rightarrow H\right) \cap \operatorname{ker}\left(G_{F\left(\zeta_{l}\right)} \rightarrow H^{\prime}\right)=K_{\cap}=\left.\operatorname{ker} \operatorname{ad} \bar{r}^{\prime \prime}\right|_{G_{F\left(\zeta_{l}\right)}}
\end{aligned}
$$

and hence there is an isomorphism $H^{\prime \prime} \cong G_{F\left(\zeta_{l}\right)} /\left.\operatorname{ker} \operatorname{ad} \bar{r}^{\prime \prime}\right|_{G_{F\left(\zeta_{l}\right)}} \cong\left\{\left(h, h^{\prime}\right) \in H \times\right.$ $\left.H^{\prime} \mid \pi(h)=\pi^{\prime}\left(h^{\prime}\right)\right\}$.

Let $K^{\prime}$ denote the kernel of $\pi^{\prime}$. It is the case that:

- The image of the inertia group at any prime above $q$ in $H^{\prime}$ is contained in $K^{\prime}$ (as $\bar{r}$ is unramified above $q$ ).

- $K^{\prime} \hookrightarrow H^{\prime} \rightarrow \operatorname{Gal}\left(M\left(\zeta_{l}\right) / F\left(\zeta_{l}\right)\right)$ is surjective (since $M$ is linearly disjoint from $\bar{F}^{\text {ker } \bar{r}}\left(\zeta_{l}\right)$ over $\left.F\right)$.

From this we easily see that $\left(\operatorname{ad} \bar{r}^{\prime \prime}\right)^{K^{\prime}}=\operatorname{ad} \bar{r}$ (use the decomposition (4.1.3)), and hence (by inflation-restriction, using the fact that $l \nmid \# K^{\prime}$, so that the group $H^{1}\left(K^{\prime}, \operatorname{ad}^{0} \bar{r}^{\prime \prime}\right)$ vanishes, and the assumption that $\left.\bar{r}\right|_{G_{F\left(\zeta_{l}\right)}}$ has big image) that:

$$
(0)=H^{1}\left(H, \operatorname{ad}^{0} \bar{r}\right)=H^{1}\left(H^{\prime \prime} / K^{\prime},\left(\operatorname{ad}^{0} \bar{r}^{\prime \prime}\right)^{K^{\prime}}\right) \stackrel{\sim}{\rightarrow} H^{1}\left(H^{\prime \prime}, \operatorname{ad}^{0} \bar{r}^{\prime \prime}\right) .
$$

All that still remains is to show the non-group-cohomology-related part of the definition of 'big image'. To this end, let us fix a copy $V_{j} \subset$ ad $\bar{r}$. (Note that every copy of $V_{j}(\chi) \subset \operatorname{ad} \bar{r}^{\prime \prime}$ will be of the form $V_{j} \otimes W_{\chi}$ for some copy of $V_{j} \subset \operatorname{ad} \bar{r}$; this uses our analysis, above, of the conditions under which terms in the direct sum (4.1.3) are isomorphic.) Since $\left.r\right|_{G_{F\left(\zeta_{l}\right)}}$ has $m^{*}$-big image, hence big image, and $M$ is linearly disjoint from $\bar{F}^{\text {ker } \bar{r}}\left(\zeta_{l}\right)$ over $F$, we see that $\left.r\right|_{G_{M\left(\zeta_{l}\right)}}$ has big image. Thus we can find a $\sigma \in G_{M\left(\zeta_{l}\right)}$ and a simple root $\alpha$ of the characteristic polynomial $\operatorname{det}(X-\bar{r}(\sigma))$ such that $\pi_{\bar{r}(\sigma), \alpha} V_{j} i_{\bar{r}(\sigma), \alpha} \neq(0)$. Altering $\sigma$ by elements of inertia subgroups at primes above $q$ (which does not affect $\bar{r}(\sigma)$ ), we can assume 2 that for $i=1, \ldots, m^{*}-1$ the ratio $\left(\bar{\theta}^{\prime} / \bar{\theta}^{\prime} \tilde{\tau}^{i}\right)(\sigma)$ does not equal $\alpha^{\prime} / \alpha$ for any root $\alpha^{\prime}$ (including $\alpha$ ) of the characteristic polynomial $\operatorname{det}(X-\bar{r}(\sigma)$ ). (That this is possible relies on the fact that $q>2 n+1$.) Thus $\alpha \bar{\theta}^{\prime}(\sigma)$ is a simple root of the characteristic polynomial of $\bar{r}^{\prime \prime}(\sigma)$ and, for each $\chi$,

$$
\begin{aligned}
& \pi_{\bar{r}^{\prime \prime}(\sigma), \alpha \bar{\theta}^{\prime}(\sigma)} \circ V_{j}(\chi) \circ i_{\bar{r}^{\prime \prime}(\sigma), \alpha \bar{\theta}^{\prime}(\sigma)} \\
& \quad=\left(\pi_{\bar{r}(\sigma), \alpha} \circ V_{j} \circ i_{\bar{r}(\sigma), \alpha}\right)\left(\pi_{\bar{r}^{\prime}(\sigma), \bar{\theta}^{\prime}(\sigma)} \circ\left(\sum_{i=0}^{m^{*}-1} \chi^{-1}\left(\tau^{i}\right) e_{i} \otimes f_{i}\right) \circ i_{\bar{r}^{\prime}(\sigma), \bar{\theta}^{\prime}(\sigma)}\right) \\
& \quad=\pi_{\bar{r}(\sigma), \alpha} \circ V_{j} \circ i_{\bar{r}(\sigma), \alpha} \neq(0) .
\end{aligned}
$$

Next, let us fix $j \in\{0, \ldots, s\}$ and $i \in\left\{1, \ldots, m^{*} / 2\right\}$, and let $\gamma: W_{i} \stackrel{\sim}{\rightarrow} W_{m^{*}-i}$ be the isomorphism such that $\gamma\left(e_{0} \otimes f_{i}\right)=e_{\left(m^{*} / 2\right)+i} \otimes f_{\left(m^{*} / 2\right)}$. (In the special case

\footnotetext{
${ }^{2}$ In particular, we note that we can alter the quantities ${\overline{\theta^{\prime}}}^{\tilde{\tau}^{i}}(\sigma)$ for $1 \leq i \leq\left(m^{*} / 2\right)-1$ independently of each other, and of $\overline{\theta^{\prime}}(\sigma)$, since altering $\sigma$ by inertia at a prime $\mathfrak{Q}^{\tau^{i}}$ will only affect $\bar{\theta}^{\prime} \tilde{\tau}^{i}(\sigma)$ and $\bar{\theta}^{\prime} \tilde{\tau}^{i+\left(m^{*} / 2\right)}(\sigma)$. Moreover, since $\left(\bar{\theta}^{\prime} / \bar{\theta}^{\prime} \tilde{\tau}^{i}\right)(\sigma)$ and $\left(\bar{\theta}^{\prime} / \bar{\theta}^{\sigma^{i}} \tilde{\tau}^{i+\left(m^{*} / 2\right)}\right)(\sigma)$ are related via the fact that $\left(\bar{\theta}^{\prime}\right)\left(\bar{\theta}^{\prime}\right)^{c}$ is unramified at all places of $M$ above $q$, we may ensure that $\left(\bar{\theta}^{\prime} / \bar{\theta}^{\tilde{\tau}^{i+\left(m^{*} / 2\right)}}\right)(\sigma)$ avoids taking the value $\alpha^{\prime} / \alpha$ by ensuring that $\bar{\theta}^{\prime} \tilde{\tau}^{i}(\sigma)$ avoids the value $\left(\alpha^{\prime}\left(\bar{\theta}^{\prime} \bar{\theta}^{\prime c}\right)(\sigma)\right) /\left(\overline{\theta^{\prime}}(\sigma) \alpha\right)$.
} 
$2 i=m^{*}, \gamma$ will happen to be the identity; we will in fact not make use of $\gamma$ in this case.) We can write any submodule of ad $\left.\bar{r}^{\prime \prime}\right|_{G_{F\left(\zeta_{l}\right)}}$ which is isomorphic to $V_{j} \otimes W_{i}$ as:

$$
\left\{\eta_{1}(v) \otimes w+\eta_{2}(v) \otimes \gamma(w): v \in V_{j}, w \in W_{i}\right\}
$$

where $\eta_{1}, \eta_{2}$ are embeddings $V_{j} \hookrightarrow$ ad $\bar{r}$, and where we suppress the second term in the sum if $2 i=m^{*}$. (This uses our analysis, above, of the conditions under which terms in the direct sum (4.1.3) are isomorphic.) Using the fact that $\left.r\right|_{G_{F\left(\zeta_{l}\right)}}$ is $m^{*}$-big, we can find a $\sigma \in G_{F\left(\zeta_{l}\right)}$ and a root $\alpha$ of $\operatorname{det}(X-\bar{r}(\sigma))$ such that:

- $\pi_{\bar{r}(\sigma), \alpha} \circ V_{j} \circ i_{\bar{r}(\sigma), \alpha} \neq(0)$.

- No other root of $\operatorname{det}(X-\bar{r}(\sigma))$ has $m^{*}$ th power equal to $\alpha^{m^{*}}$.

Since $M$ is linearly disjoint from $\bar{F}^{\text {ker } \bar{r}}\left(\zeta_{l}\right)$ over $F$, we may additionally assume:

- $\sigma$ maps to the generator $\tau$ of $\operatorname{Gal}\left(M\left(\zeta_{l}\right) / F\left(\zeta_{l}\right)\right)$.

Define $\beta_{0}, \ldots, \beta_{m^{*}-1}$ by

$$
\bar{r}^{\prime}(\sigma) e_{i}=\beta_{i} e_{i+1}
$$

(where we take subscripts modulo $m^{*}$ ). The roots of the characteristic polynomial of $\bar{r}^{\prime}(\sigma)$ are exactly the $m^{*}$ th roots of $\beta_{0} \beta_{1} \ldots \beta_{m^{*}-1}$. If $\beta$ is such a root, so $\beta^{m^{*}}=\beta_{0} \beta_{1} \ldots \beta_{m^{*}-1}$, then a corresponding eigenvector is

$$
v_{\beta}:=e_{0}+\frac{\beta_{0}}{\beta} e_{1}+\frac{\beta_{0} \beta_{1}}{\beta^{2}} e_{2}+\cdots+\frac{\beta_{0} \ldots \beta_{m^{*}-2}}{\beta^{m^{*}-1}} e_{m^{*}-1}
$$

and the corresponding equivariant projection is

$$
\pi_{\bar{r}^{\prime}(\sigma), \beta} e_{j}=\frac{\beta^{j}}{m^{*} \beta_{0} \beta_{1} \ldots \beta_{j-1}} v_{\beta} .
$$

We see that $\alpha \beta$ is a simple root of the characteristic polynomial $\operatorname{det}\left(X-\bar{r}^{\prime \prime}(\sigma)\right)$, and that for $v \in V_{j}$,

$$
\begin{aligned}
& \pi_{\bar{r}^{\prime \prime}(\sigma), \alpha \beta} \circ\left(\eta_{1}(v) \otimes e_{0} \otimes f_{i}+\eta_{2}(v) \otimes e_{\left(m^{*} / 2\right)+i} \otimes f_{\left(m^{*} / 2\right)}\right) \circ i_{\bar{r}^{\prime \prime}(\sigma), \alpha \beta} \\
& =\left(\pi_{\bar{r}(\sigma), \alpha} \circ \eta_{1}(v) \circ i_{\bar{r}(\sigma), \alpha}\right)\left(\pi_{\bar{r}^{\prime}(\sigma), \beta} \circ\left(e_{0} \otimes f_{i}\right) \circ i_{\bar{r}^{\prime}(\sigma), \beta}\right) \\
& \quad+\left(\pi_{\bar{r}(\sigma), \alpha} \circ \eta_{2}(v) \circ i_{\bar{r}(\sigma), \alpha}\right)\left(\pi_{\bar{r}^{\prime}(\sigma), \beta} \circ\left(e_{\left(m^{*} / 2\right)+i} \otimes f_{\left(m^{*} / 2\right)}\right) \circ i_{\bar{r}^{\prime}(\sigma), \beta}\right) \\
& =\left(\pi_{\bar{r}(\sigma), \alpha} \circ \eta_{1}(v) \circ i_{\bar{r}(\sigma), \alpha}\right) \frac{1}{m^{*}} \frac{\beta_{0} \ldots \beta_{i-1}}{\beta^{i}} \\
& \quad+\left(\pi_{\bar{r}(\sigma), \alpha} \circ \eta_{2}(v) \circ i_{\bar{r}(\sigma), \alpha}\right) \frac{\beta^{m^{*} / 2+i}}{m^{*} \beta_{0} \ldots \beta_{m^{*} / 2+i-1}} \frac{\beta_{0} \ldots \beta_{m^{*} / 2-1}}{\beta^{m^{*} / 2}} \\
& =\frac{1}{m^{*}}\left(\left(\pi_{\bar{r}(\sigma), \alpha} \circ \eta_{1}(v) \circ i_{\bar{r}(\sigma), \alpha}\right) \frac{\beta_{0} \ldots \beta_{i-1}}{\beta^{i}}\right) . \\
& \left.\quad+\left(\pi_{\bar{r}(\sigma), \alpha} \circ \eta_{2}(v) \circ i_{\bar{r}(\sigma), \alpha}\right) \frac{\beta^{i}}{\beta_{m^{*} / 2} \ldots \beta_{m^{*} / 2+i-1}}\right) .
\end{aligned}
$$

This will be non-zero for some choice of $\beta$ and $v$.

Since all terms in the sum (4.1.3) are isomorphic either to a $V_{j}(\chi)$ or a $W_{i} \otimes V_{j}$, the only remaining point is to check that $\bar{r}^{\prime \prime}\left(G_{F\left(\zeta_{l}\right)}\right)$ has no quotients of $l$-power order. It suffices to prove that $H^{\prime \prime}=\left(\operatorname{ad} \bar{r}^{\prime \prime}\right)\left(G_{F\left(\zeta_{l}\right)}\right)$ has no quotients of $l$-power order (because the group of scalar matrices in $\mathrm{GL}_{n m^{*}}\left(\overline{\mathbb{F}}_{l}\right)$ has no elements of order divisible by $l$ ). Since $m^{*}$ is not divisible by $l, H^{\prime}$ has order prime to $l$, and we see that any quotient of $H^{\prime \prime}$ of $l$-power order would also be a quotient of $H$. Since $\left.r\right|_{G_{F\left(\zeta_{l}\right)}}$ has $m^{*}$-big image, $H$ has no quotients of $l$-power order, and we are done. 
4.2. We now return to the main business of section: constructing characters. For the entire remainder of this section, we will be working with the following combinatorial data in the background:

Situation 4.2.1. Suppose that $F$ is a totally real field, $l$ is a rational prime which splits completely in $F$, and that we are given the following data:

- a partition of the set of primes above $l$ into two subsets $S_{\text {ord }}$ and $S_{\text {ss }}$,

- for each prime $v$ above $l$, integers $a_{v}$ and $b_{v}$, and

- a set $T$ of places of $F$, not containing places above $l$

such that the sum $-2 a_{v}+b_{v}$ takes some fixed value, $w$ say, independent of $v$.

(It may be helpful to the reader if we remark that this combinatorial data is intended to be related to the automorphic representation $\pi$ of $\mathrm{GL}_{2}\left(\mathbb{A}_{F}\right)$ with which we will eventually be working in the following manner: the sets $S_{\text {ord }}, S_{\text {ss }}$ reflect the places above $l$ where $\pi$ is ordinary and where it is supersingular, $\pi$ is thought of as being associated to a Galois representation having Hodge-Tate numbers $\left\{-a_{v}, b_{v}-\right.$ $\left.a_{v}\right\}$ at the place $v$, and the set $T$ contains the places away from $l$ where $\pi$ is ramified.)

We define a certain integer $m^{*}$, dependent on the set of $b_{v}$ 's of Situation 4.2.1. but not on the prime $l$ itself.

Definition 4.2.2. Let $B=\left\{b_{v} \mid v\right.$ a prime of $F$ above $\left.l\right\}$, considered as a set without multiplicity. We define the integer $m^{*}$ to be the least common multiple of the integers in the set $\{2\} \cup B$.

We have seen in the previous section that our lifting theorems require us to maintain careful control of the lattices with which we work. We therefore single out certain lattices which will be important in the sequel.

Definition 4.2.3. Suppose we are in the situation of Situation 4.2.1. We make the following definitions:

(1) Suppose that $v \in S_{\mathrm{ss}}$ is a place of $F$ above $l$, and let $L$ be the quadratic unramified extension of $F_{v}$ in $\bar{F}_{v}$ (so that $L$ is isomorphic to $\mathbb{Q}_{l^{2}}$ ). Let $K$ be a finite extension of $\mathbb{Q}_{l}$ with ring of integers $\mathcal{O}$, and suppose that $\chi: G_{L} \rightarrow \mathcal{O}^{\times}$is a de Rham character. Finally suppose we have chosen $\sigma \in G_{F_{v}}$ mapping to a generator of $\operatorname{Gal}\left(L / F_{v}\right)$. Then we can consider the ordered $\mathcal{O}$-basis $\left\{f_{0}, f_{1}\right\}$ of $\operatorname{Ind}_{G_{L}}^{G_{F_{v}}} \chi$, where $f_{i}: G_{F_{v}} \rightarrow \mathcal{O}$ is the function supported on $\sigma^{i} G_{L}$ and taking the value 1 on $\sigma^{i}$. We call this the $\sigma$-standard basis for $\operatorname{Ind}_{G_{L}}^{G_{F_{v}}} \chi$.

(2) Continue the assumptions of the previous point. We get an ordered basis $\left\{g_{0}, \ldots, g_{n-1}\right\}$ of $\operatorname{Sym}^{n-1} \operatorname{Ind}_{G_{L}}^{G_{F_{v}}} \chi$ inherited (in the sense of Definition 3.3.1) from the $\sigma$-standard basis on $\operatorname{Ind}_{G_{L}}^{G_{F}} \chi$ defined in the previous part. We call this the $\sigma$-standard basis of $\operatorname{Sym}^{n-1} \operatorname{Ind}_{G_{L}}^{G_{F_{v}}} \chi$. (Concretely, considering $\operatorname{Sym}^{n-1} \operatorname{Ind}_{G_{L}}^{G_{F_{v}}} \chi$ as a quotient of $\left(\operatorname{Ind}_{G_{L}}^{G_{F_{v}}} \chi\right)^{\otimes(n-1)}, g_{i}$ is the image of $f_{0}^{\otimes(n-1-i)} \otimes f_{1}^{\otimes i}$.)

(3) Suppose $M / F$ is a cyclic degree $m^{*}$ extension with $F$ totally real and $M$ $\mathrm{CM}, K$ is a finite extension of $\mathbb{Q}_{l}$ with ring of integers $\mathcal{O}, \widetilde{\tau} \in G_{F}$ an element mapping to a generator $\tau \in \operatorname{Gal}(M / F)$, and $\theta: G_{M} \rightarrow \mathcal{O}^{\times}$is a character. We consider the ordered $\mathcal{O}$-basis $\left\{e_{0}, \ldots, e_{m^{*}-1}\right\}$ of $\operatorname{Ind}_{G_{M}}^{G_{F}} \theta$, 
where $e_{i}: G_{F} \rightarrow \mathcal{O}$ is the function supported on $\widetilde{\tau}^{i} G_{M}$ with value 1 on $\widetilde{\tau}^{i}$, and call this the $\widetilde{\tau}$-standard basis for $\operatorname{Ind}_{G_{M}}^{G_{F}} \theta$.

(4) If $v, L, K, M, \chi, \theta, \sigma, \widetilde{\tau}$ are as above, then we consider $\operatorname{Sym}^{n-1} \operatorname{Ind}_{G_{L}}^{G_{F_{v}}} \chi \otimes$ $\operatorname{Ind}_{G_{M}}^{G_{F}} \theta$, which is a representation of $G_{F_{v}}$, and has an ordered basis inherited (in the sense of Definition 3.3.2) from the $\sigma$-standard and $\widetilde{\tau}$-standard bases on $\operatorname{Sym}^{n-1} \operatorname{Ind}_{G_{L}}^{G_{F_{v}}} \chi$ and $\operatorname{Ind}_{G_{M}}^{G_{F}} \theta$ already defined. We call this the $(\sigma, \widetilde{\tau})$-standard basis of $\operatorname{Sym}^{n-1} \operatorname{Ind}_{G_{L}}^{G_{F}} \chi \otimes \operatorname{Ind}_{G_{M}}^{G_{F}} \theta$.

We are now in a position to construct the characters we will need.

Lemma 4.2.4. Suppose we are in the situation described in Situation 4.2.1, and we have fixed an integer $n$ and an extension $F^{(\mathrm{bad})}$ of $F$. Assume that $l \nmid m^{*}$ and $l>2 n-2$. Then we can find a degree $m^{*}$ cyclic $C M$ extension $M$ of $F$, linearly disjoint from $F^{\text {(bad) }}$ over $F$, and continuous characters

$$
\theta, \theta^{\prime}: G_{M} \rightarrow \overline{\mathbb{Z}}_{l}^{\times},
$$

which are de Rham at all primes above l, and which enjoy the following further properties:

(1) $\theta, \theta^{\prime}$ are congruent $(\bmod l)$.

(2) Suppose that $v \in S_{\mathrm{ss}}$ is a place of $F$ above $l$, and let $L$ be the quadratic unramified extension of $F_{v}$ in $\bar{F}_{v}$ (so that $L$ is isomorphic to $\mathbb{Q}_{l^{2}}$ ). Suppose that $\chi, \chi^{\prime}: G_{L} \rightarrow \overline{\mathbb{Q}}_{l}^{\times}$are de Rham characters with $\bar{\chi}=\bar{\chi}^{\prime}$. Suppose furthermore that the Hodge-Tate weights of $\chi$ are $-a_{v}$ and $b_{v}-a_{v}$, while those of $\chi^{\prime}$ are 0 and 1.

Let $K \subset \overline{\mathbb{Q}}_{l}$ be a finite extension of $\mathbb{Q}_{l}$ with ring of integers $\mathcal{O}$, and suppose that $K$ is large enough that $\theta, \theta^{\prime}, \chi$ and $\chi^{\prime}$ are all valued in $\mathcal{O}$. Let $\sigma \in G_{F_{v}}$ be an element mapping to a generator of $\operatorname{Gal}\left(L / F_{v}\right)$, and $\widetilde{\tau} \in G_{F}$ an element mapping to a generator $\tau \in \operatorname{Gal}(M / F)$.

Let $L^{\prime}$ be a finite extension of $L$ in $\bar{F}_{v}$ such that $\left.\chi\right|_{G_{L^{\prime}}}, \chi_{G_{L^{\prime}}}^{\prime},\left.\theta\right|_{G_{L^{\prime}}}$, and $\left.\theta^{\prime}\right|_{G_{L^{\prime}}}$ are all crystalline. Let

$$
\begin{aligned}
\rho_{\chi} & =\left.\left.\left(\operatorname{Sym}^{n-1} \operatorname{Ind}_{G_{L}}^{G_{F_{v}}} \chi\right)\right|_{G_{L^{\prime}}} \otimes\left(\operatorname{Ind}_{G_{M}}^{G_{F}} \theta\right)\right|_{G_{L^{\prime}},}, \\
\text { and } \rho_{\chi^{\prime}} & =\left.\left.\left(\operatorname{Sym}^{n-1} \operatorname{Ind}_{G_{L}}^{G_{F_{v}}} \chi^{\prime}\right)\right|_{G_{L^{\prime}}} \otimes\left(\operatorname{Ind}_{G_{M}}^{G_{F}} \theta^{\prime}\right)\right|_{G_{L^{\prime}}},
\end{aligned}
$$

regarded as representations $G_{L^{\prime}} \rightarrow \mathrm{GL}_{n m^{*}}(\mathcal{O})$ with respect to their $(\sigma, \widetilde{\tau})$ standard bases. Note that $\rho_{\chi}$ and $\rho_{\chi^{\prime}}^{\prime}$ become equal after composition with the homomorphism $\mathrm{GL}_{n m^{*}}(\mathcal{O}) \rightarrow \mathrm{GL}_{n m^{*}}(k)$. Assume in fact that $L^{\prime}$ has been chosen so that this common composite is the trivial representation.

Then $\rho_{\chi} \sim \rho_{\chi^{\prime}}$ (in the sense of Definition 3.3.5).

(3) For any $r: \operatorname{Gal}(\bar{F} / F) \rightarrow \mathrm{GL}_{n}\left(\overline{\mathbb{Z}}_{l}\right)$, a continuous Galois representation ramified only at primes in $T$ and above l, which satisfies $\bar{F}^{\mathrm{ker} \bar{r}}\left(\zeta_{l}\right) \subset F^{(\mathrm{bad})}$ :

- If $r\left(G_{F\left(\zeta_{l}\right)}\right)$ is $m^{*}$-big, then the images of $\left.\left(r \otimes \operatorname{Ind}_{G_{M}}^{G_{F}} \theta\right)\right|_{G_{F\left(\zeta_{l}\right)}}$ and $\left.\left(r \otimes \operatorname{Ind}_{G_{M}}^{G_{F}} \theta^{\prime}\right)\right|_{G_{F\left(\zeta_{l}\right)}}$ are big.

- If $\left[\bar{F}^{\operatorname{kerad} \bar{r}}\left(\zeta_{l}\right): \bar{F}^{\text {ker ad } \bar{r}}\right]>m^{*}$, then neither the fixed field of the kernel of $\operatorname{ad}\left(\bar{r} \otimes \operatorname{Ind}_{G_{M}}^{G_{F}} \bar{\theta}\right)$ nor that of $\operatorname{ad}\left(\bar{r} \otimes \operatorname{Ind}_{G_{M}}^{G_{F}} \bar{\theta}^{\prime}\right)$ will contain $\zeta_{l}$.

(4) We can put a perfect pairing on $\operatorname{Ind}_{G_{M}}^{G_{F}} \theta$ satisfying:

(a) $\left\langle v_{1}, v_{2}\right\rangle=(-1)^{n}\left\langle v_{2}, v_{1}\right\rangle$. 
(b) For $\sigma \in G_{F}$, we have

$$
\left\langle\sigma v_{1}, \sigma v_{2}\right\rangle=\epsilon_{l}(\sigma)^{-m^{*} n+1-(1-n) w} \tilde{\omega}(\sigma)^{-(w-1)(n-1)}\left\langle v_{1}, v_{2}\right\rangle,
$$

where $\tilde{\omega}$ is the Teichmüller lift of the mod l cyclotomic character.

Thus, in particular,

$$
\left(\operatorname{Ind}_{G_{M}}^{G_{F}} \theta\right)^{\vee} \cong\left(\operatorname{Ind}_{G_{M}}^{G_{F}} \theta\right) \otimes \epsilon_{l}^{m^{*} n-1+(1-n) w} \tilde{\omega}^{(w-1)(n-1)} .
$$

(Note that the character on the right hand side takes the value $(-1)^{n}$ on complex conjugations.)

(5) Similarly, we can put a perfect pairing on $\operatorname{Ind}_{G_{M}}^{G_{F}} \theta^{\prime}$ satisfying:

(a) $\left\langle v_{1}, v_{2}\right\rangle=(-1)^{n}\left\langle v_{2}, v_{1}\right\rangle$.

(b) For $\sigma \in G_{F}$, we have $\left\langle\sigma v_{1}, \sigma v_{2}\right\rangle=\epsilon_{l}(\sigma)^{-\left(m^{*}-1\right) n}\left\langle v_{1}, v_{2}\right\rangle$.

Thus, in particular, $\left(\operatorname{Ind}_{G_{M}}^{G_{F}} \theta^{\prime}\right)^{\vee} \cong\left(\operatorname{Ind}_{G_{M}}^{G_{F}} \theta^{\prime}\right) \otimes \epsilon_{l}^{\left(m^{*}-1\right) n}$.

(Again the character on the right hand side is $(-1)^{n}$ on complex conjugations.)

(6) Suppose $r: \operatorname{Gal}(\bar{F} / F) \rightarrow \mathrm{GL}_{2}\left(\overline{\mathbb{Z}}_{l}\right)$ is a continuous representation with Hodge-Tate weights $\left\{-a_{v}, b_{v}-a_{v}\right\}$ at $v$ for each place $v$ of $F$ above $l$; then $\operatorname{Sym}^{n-1} r \otimes \operatorname{Ind}_{G_{M}}^{G_{F}} \theta$ has the following Hodge-Tate weights at $v$ :

$$
\left\{0,1,2, \ldots, m^{*} n-2, m^{*} n-1\right\}
$$

(for each $v$ ). In particular, $\operatorname{Sym}^{n} r \otimes \operatorname{Ind}_{G_{M}}^{G_{F}} \theta$ is regular.

(7) Suppose $r^{\prime}: \operatorname{Gal}(\bar{F} / F) \rightarrow \mathrm{GL}_{2}\left(\overline{\mathbb{Z}}_{l}\right)$ is a continuous representation with Hodge-Tate weights $\{0,1\}$ at $v$ for each place $v$ of $F$ above l; then $\mathrm{Sym}^{n-1} r^{\prime}$ $\otimes \operatorname{Ind}_{G_{M}}^{G_{F}} \theta^{\prime}$ also has the following Hodge-Tate weights at $v$ :

$$
\left\{0,1,2, \ldots, m^{*} n-2, m^{*} n-1\right\}
$$

(for each $v$ ). In particular, $\operatorname{Sym}^{n} r^{\prime} \otimes \operatorname{Ind}_{G_{M}}^{G_{F}} \theta^{\prime}$ is regular.

Proof.

Step 1. Finding a suitable field $M$. We claim that there exists a surjective character $\chi: \operatorname{Gal}(\bar{F} / F) \rightarrow \mu_{m^{*}}\left(\right.$ where $\mu_{m^{*}}$ is the group of $m^{*}$-th roots of unity in $\left.\overline{\mathbb{Q}}^{\times}\right)$such that:

- $\chi$ is unramified at all places of $F$ above $l$.

- $\chi\left(\operatorname{Frob}_{v}\right)=1$ for all $v \in S_{\text {ord }}$.

- $\chi\left(\operatorname{Frob}_{v}\right)=-1$ for all $v \in S_{\mathrm{ss}}$.

- $\chi\left(c_{v}\right)=-1$ for each infinite place $v$ (where $c_{v}$ denotes a complex conjugation at $v)$.

- $\bar{F}^{\text {ker } \chi}$ is linearly disjoint from $F^{\text {(bad) }}$ over $F$.

We construct the character $\chi$ as follows. First, we find using weak approximation a totally negative element $\alpha \in F^{\times}$which is a $v$-adic unit for each $v \mid l$, and which is congruent to a quadratic residue mod each $v \in S_{\text {ord }}$ and a quadratic non-residue $\bmod$ each $v \in S_{\mathrm{ss}}$. Let $\chi_{0}$ be the quadratic character associated to the extension we get by adjoining the square root of this element. Then:

- $\chi_{0}$ is unramified at all places of $F$ above $l$.

- $\chi_{0}\left(\right.$ Frob $\left._{v}\right)=1$ for all $v \in S_{\text {ord }}$.

- $\chi_{0}\left(\right.$ Frob $\left._{v}\right)=-1$ for all $v \in S_{\mathrm{ss}}$. 
- $\chi_{0}\left(c_{v}\right)=-1$ for each infinite place $v$ (where $c_{v}$ denotes a complex conjugation at $v)$.

Now choose (for example by Theorem 6 of chapter 10 of [AT09]) a cyclic totally real extension $M_{1} / \mathbb{Q}$ of degree $m^{*}$ such that:

- $M_{1} / \mathbb{Q}$ is unramified at all the rational primes where $\bar{F}^{\mathrm{ker} \chi_{0}} F^{(\mathrm{bad})} / \mathbb{Q}$ is ramified.

- $l$ splits completely in $M_{1}$.

Since $\bar{F}^{\text {ker } \chi_{0}} F^{\text {(bad) }} / \mathbb{Q}$ and $M_{1} / \mathbb{Q}$ ramify at disjoint sets of primes, they are linearly disjoint, and we can find a rational prime $p$ which splits completely in $F^{(\text {bad })} \bar{F}^{\text {ker } \chi_{0}}$ but such that $\operatorname{Frob}_{p}$ generates $\operatorname{Gal}\left(M_{1} / \mathbb{Q}\right)$. Since $M_{1} / \mathbb{Q}$ is cyclic, we may pick an isomorphism between $\operatorname{Gal}\left(M_{1} / \mathbb{Q}\right)$ and $\mu_{m^{*}}$, and we can think of $M_{1}$ as determining a character $\chi_{1}: G_{\mathbb{Q}} \rightarrow \mu_{m^{*}}$ such that:

- $\chi_{1}$ is trivial on $G_{\mathbb{Q}_{l}}$.

- $\chi_{1}$ is trivial on complex conjugation.

- $\chi_{1}\left(\operatorname{Frob}_{p}\right)=\zeta_{m^{*}}$, a primitive $m^{*}$ th root of unity.

Then, set $\chi=\left(\left.\chi_{1}\right|_{G_{F}}\right) \chi_{0}$. Note that this maps onto $\mu_{m^{*}}$, even when we restrict to $G_{F^{\text {(bad) }}}$ (since $p$ splits completely in $F^{\text {(bad) }}$ and if $\wp$ is a place of $F^{(\text {bad) }}$ over $p$, we have $\chi_{0}\left(\operatorname{Frob}_{\wp}\right)=1$ while $\left.\chi_{1}\left(\operatorname{Frob}_{\wp}\right)=\zeta_{m^{*}}\right)$. The remaining properties are clear.

Having shown that $\chi$ exists, we set $M=\bar{F}^{\text {ker } \chi}$; note that this is a CM field, and a cyclic extension of $F$ of degree $m^{*}$. Write $\tau$ for a generator of $\operatorname{Gal}(M / F)$. Write $M^{+}$for the maximal totally real subfield of $M$.

Step 2. Defining certain sequences of numbers. We will now define certain sequences of numbers. The reason for introducing them is that the characters $\theta$ and $\theta^{\prime}$ will be engineered such that their Hodge-Tate numbers at the primes above $l$ in $M$ will be given according to the sequences we are about to construct, and many of the formulae we use in defining $\theta$ and $\theta^{\prime}$ will require explicit mention of these numbers. Thus it will be helpful to have introduced notation for them.

In particular, for each prime $v$ of $F$ above $l$, we define $m^{*}$-tuples of integers $\left(h_{v, 0}, \ldots, h_{v, m^{*}-1}\right)$ and $\left(h_{v, 0}^{\prime}, \ldots, h_{v, m^{*}-1}^{\prime}\right)$ by putting:

$$
\begin{gathered}
\left(h_{v, 0}, \ldots, h_{v, m^{*}-1}\right) \\
=\left(a_{v}(n-1), 1+a_{v}(n-1), 2+a_{v}(n-1), \ldots, b_{v}-1+a_{v}(n-1),\right. \\
b_{v} n+a_{v}(n-1), b_{v} n+1+a_{v}(n-1), \ldots, b_{v} n+\left(b_{v}-1\right)+a_{v}(n-1), \\
\ldots, \\
\left(m^{*}-b_{v}\right) n+a_{v}(n-1),\left(m^{*}-b_{v}\right) n+1+a_{v}(n-1), \\
\left.\ldots,\left(m^{*}-b_{v}\right) n+b_{v}-1+a_{v}(n-1)\right), \\
\left(h_{v, 0}^{\prime}, \ldots, h_{v, m^{*}-1}^{\prime}\right)=\left(0, n, 2 n, \ldots,\left(m^{*}-1\right) n\right) .
\end{gathered}
$$

We note that, so defined, $h$ and $h^{\prime}$ satisfy, for each $i$ :

$$
h_{v, i}+h_{v, m^{*}-i-1}=\left(m^{*}-b_{v}\right) n+b_{v}-1+2 a_{v}(n-1)=m^{*} n-1+(1-n) w,
$$

$$
h_{v, i}^{\prime}+h_{v, m^{*}-i-1}^{\prime}=\left(m^{*}-1\right) n .
$$

(The characters $\theta$ and $\theta^{\prime}$ will be engineered to have these Hodge-Tate numbers at the primes above $l$ in $M$.) 
Step 3. An auxiliary prime $q$. Choose a rational prime $q$ such that

- no prime of $T$ lies above $q$,

- $q \neq l$,

- $q$ splits completely in $M$,

- $q$ is unramified in $F^{(\text {bad) }}$, and

- $q-1>2 n$.

Also choose a prime $\mathfrak{q}$ of $F$ above $q$, and a prime $\mathfrak{Q}$ of $\mathrm{M}$ above $\mathfrak{q}$.

Step 4. Defining certain algebraic characters $\phi, \phi^{\prime}: \mathbb{A}_{M}^{\times} \rightarrow\left(M^{\prime}\right)^{\times}$. For each prime $v$ of $F$ above $l$, let us choose a prime $w_{v}$ of $M$ above $v$. We now have a convenient notation for all the primes above $v$; if $v \in S_{\text {ord }}$, there are $m^{*}$ of them, $\tau^{j} w_{v}$ for $j=$ $0, \ldots, m^{*}-1$; and if $v \in S_{\mathrm{ss}}$, there are $m^{*} / 2$ of them, $\tau^{j} w_{v}$ for $j=0, \ldots,\left(m^{*} / 2\right)-1$. Also, choose $\iota_{v}$ to be an embedding $M \rightarrow \overline{\mathbb{Q}}_{l}$ attached to the prime $w_{v}$ (in case $v \in S_{\text {ord }}$ there is only one choice; in case $v \in S_{\mathrm{ss}}$ there are two).

We are now forced into a slight notational ugliness. Write $\tilde{M}$ for the Galois closure of $M$ over $\mathbb{Q}$. (Thus $\operatorname{Gal}(\tilde{M} / \mathbb{Q})$ is in bijection with embeddings $\tilde{M} \rightarrow \overline{\mathbb{Q}}$.) Let us fix $\iota^{*}$, an embedding of $\tilde{M}$ into $\overline{\mathbb{Q}}_{l}$, and write $v^{*}$ for the prime of $M$ below this 3 Given any embedding $\iota^{\prime}$ of $M$ into $\overline{\mathbb{Q}}_{l}$, we can choose an element $\sigma_{\iota^{\prime}}$ in $\operatorname{Gal}(\tilde{M} / \mathbb{Q})$ such that $\iota^{\prime}=\iota^{*} \circ \sigma_{\iota^{\prime}}$.

We claim that there exists an extension $M^{\prime}$ of $M$, and a character $\phi: \mathbb{A}_{M}^{\times} \rightarrow$ $\left(M^{\prime}\right)^{\times}$with open kernel such that:

- For $\alpha \in M^{\times}$,

$$
\phi(\alpha)=\prod_{v \in S_{\mathrm{ord}} \cup S_{\mathrm{ss}}} \prod_{j=0}^{\left(m^{*} / 2\right)-1}\left(\sigma_{\iota_{v} \circ \tau^{-j}}(\alpha)\right)^{h_{v, j}}\left(\sigma_{\iota_{v} \circ \tau^{-j-\left(m^{*} / 2\right)}}(\alpha)\right)^{h_{v, m^{*}-1-j}} .
$$

- For $\alpha \in\left(\mathbb{A}_{M^{+}}\right)^{\times}$, we have

$$
\phi(\alpha)=\left(\prod_{v \nmid \infty}\left|\alpha_{v}\right| \prod_{v \mid \infty} \operatorname{sgn}_{v}\left(\alpha_{v}\right) \delta_{M / M^{+}}\left(\operatorname{Art}_{M^{+}}(\alpha)\right)\right)^{-m^{*} n+1-(1-n) w},
$$

where $\delta_{M / M^{+}}$is the quadratic character of $G_{M^{+}}$associated to $M$. (Note that, on the right hand side, we really think of $\alpha$ as an element of $\mathbb{A}_{M^{+}}$, not just as an element of $\mathbb{A}_{M}$ which happens to lie in $\mathbb{A}_{M^{+}}$; so for instance $v$ runs over places of $M^{+}$, and the local norms are appropriately normalized to reflect our thinking of them as places of $M^{+}$.)

- $\phi$ is unramified at $l$.

This is an immediate consequence of Lemma 2.2 of HSBT10; we must simply verify that the conditions in the bullet points are compatible; the only difficult part is comparing the first and second, where equation (4.2.1) gives us what we need 4

Similarly, we construct a character $\phi^{\prime}:\left(\mathbb{A}_{M}\right)^{\times} \rightarrow\left(M^{\prime}\right)^{\times}$(enlarging $M^{\prime}$ if necessary) with open kernel such that:

\footnotetext{
${ }^{3}$ The choice of this $\iota^{*}$ will affect the choice of the algebraic characters $\phi, \phi^{\prime}$ below, but will be cancelled out - at least concerning the properties we care about - when we pass to the $l$-adic characters $\theta, \theta^{\prime}$ below.

${ }^{4}$ We also use the fact that, if we fix a complex embedding $\iota_{\mathbb{C}}$ of $M, \iota \mathbb{C} \circ \sigma_{\iota v} \circ \tau^{j}$ will run through all other complex embeddings as $v$ runs through primes above $l$ and $j$ runs from 0 to $m^{*}-1$, as may be seen by taking a field isomorphism $\mathbb{C} \cong \overline{\mathbb{Q}}_{l}$.
} 
- For $\alpha \in M^{\times}$,

$$
\phi^{\prime}(\alpha)=\prod_{v \in S_{\mathrm{ord}} \cup S_{\mathrm{ss}}} \prod_{j=0}^{\left(m^{*} / 2\right)-1}\left(\sigma_{\iota_{v} \circ \tau^{-j}}(\alpha)\right)^{h_{v, j}^{\prime}}\left(\sigma_{\iota_{v} \circ \tau^{-j-\left(m^{*} / 2\right)}}(\alpha)\right)^{h_{v, m^{*}-1-j}^{\prime}}
$$

- For $\alpha \in\left(\mathbb{A}_{M^{+}}\right)^{\times}$, we have

$$
\phi^{\prime}(\alpha)=\left(\prod_{v \nmid \infty}\left|\alpha_{v}\right| \prod_{v \mid \infty} \operatorname{sgn}_{v}\left(\alpha_{v}\right) \delta_{M / M^{+}}\left(\operatorname{Art}_{M^{+}}(\alpha)\right)\right)^{-\left(m^{*}-1\right) n} .
$$

(Again, we think of $\alpha$ on the right hand side as a bona fide member of $\mathbb{A}_{M^{+}}$.)

- $\phi^{\prime}$ is unramified at $l$.

- $q \mid \# \phi^{\prime}\left(\mathcal{O}_{M, \mathfrak{Q}}^{\times}\right)$, but $\phi^{\prime}$ is unramified at primes above $\mathfrak{q}$ other than $\mathfrak{Q}$ and $\mathfrak{Q}^{c}$. Again, this follows from Lemma 2.2 of HSBT10, now using equation (4.2.2).

Step 5. Defining the characters $\theta, \theta^{\prime}: \operatorname{Gal}(\bar{M} / M) \rightarrow \overline{\mathbb{Z}}_{l}^{\times}$. Write $\tilde{M}^{\prime}$ for the Galois closure of $M^{\prime}$ over $\mathbb{Q}$, and extend $\iota^{*}: \tilde{M} \rightarrow \overline{\mathbb{Q}}_{l}$ to an embedding $\iota^{* *}: \tilde{M}^{\prime} \rightarrow \overline{\mathbb{Q}}_{l}$. Define $l$-adic characters $\theta_{0}, \theta^{\prime}: \operatorname{Gal}(\bar{M} / M) \rightarrow \overline{\mathbb{Z}}_{l}^{\times}$by

$$
\begin{aligned}
& \theta_{0}(\operatorname{Art} \alpha)=\iota^{* *}(\phi(\alpha)) \prod_{v \mid l} \prod_{j=0}^{\left(m^{*} / 2\right)-1}\left(\iota_{v} \circ \tau^{-j}\right)\left(\alpha_{\tau^{j} w_{v}}\right)^{-h_{v, j}}\left(\iota_{v} \circ \tau^{-j-\left(m^{*} / 2\right)}\right) \\
& \times\left(\alpha_{\tau^{\left(m^{*} / 2\right)+j} w_{v}}\right)^{-h_{v, m^{*}-1-j}},
\end{aligned}
$$

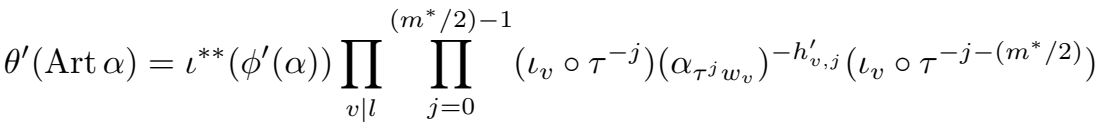

$$
\begin{aligned}
& \times\left(\alpha_{\tau^{\left(m^{*} / 2\right)+j} w_{v}}\right)^{-h_{v, m^{*}-1-j}^{\prime}},
\end{aligned}
$$

where $v$ runs over places of $F$ dividing $l$. (It is easy to check that the expressions on the right hand sides are unaffected when $\alpha$ is multiplied by an element of $M^{\times}$.) Observe then that they enjoy the following properties:

- $\theta^{\prime} \circ V_{M / M^{+}}=\left(\epsilon_{l} \delta_{M / M^{+}}\right)^{-\left(m^{*}-1\right) n}$, where $V_{M / M^{+}}$is the transfer map $G_{M^{+}}^{\mathrm{ab}} \rightarrow$ $G_{M}^{\mathrm{ab}}$. In particular, $\theta^{\prime} \theta^{\prime c}=\epsilon_{l}^{-\left(m^{*}-1\right) n}$.

- $\theta_{0} \circ V_{M / M^{+}}=\left(\epsilon_{l} \delta_{M / M^{+}}\right)^{-m^{*} n+1-(1-n) w}$ and hence, $\theta_{0} \theta_{0}^{c}=\epsilon_{l}^{-m^{*} n+1-(1-n) w}$.

- For $v \in S_{\text {ord }}$ and $0 \leq j \leq\left(m^{*} / 2\right)-1$, the Hodge-Tate weight of $\left.\theta_{0}\right|_{G_{M_{\tau} j_{w}}}$ is $h_{v, j}$, and the Hodge-Tate weight of $\left.\theta_{0}\right|_{G_{M_{\tau} j+m^{*} / 2 w_{v}}}$ is $h_{v, m^{*}-1-j}$.

- For $v \in S_{\mathrm{ss}}$ and $0 \leq j \leq\left(m^{*} / 2\right)-1$, the Hodge-Tate weights of $\left.\theta_{0}\right|_{G_{M_{\tau^{j}} w_{v}}}$ are $h_{v, j}$ and $h_{v, m^{*}-1-j}$.

- For $v \in S_{\text {ord }}$ and $0 \leq j \leq\left(m^{*} / 2\right)-1$, the Hodge-Tate weight of $\left.\theta^{\prime}\right|_{G_{M_{\tau^{j}} w_{v}}}$

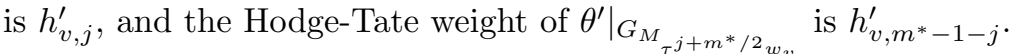

- For $v \in S_{\mathrm{ss}}$ and $0 \leq j \leq\left(m^{*} / 2\right)-1$, the Hodge-Tate weights of $\left.\theta^{\prime}\right|_{G_{M_{\tau^{j}} w_{v}}}$ are $h_{v, j}^{\prime}$ and $h_{v, m^{*}-1-j}^{\prime}$.

- $q \mid \# \theta^{\prime}\left(I_{\mathfrak{Q}}\right)$, but $\theta^{\prime}$ is unramified at all primes above $\mathfrak{q}$ except $\mathfrak{Q}, \mathfrak{Q}^{c}$.

We now define $\theta=\theta_{0}\left(\tilde{\theta^{\prime}} / \tilde{\theta_{0}}\right)$, where $\tilde{\theta_{0}}\left(\operatorname{resp} \tilde{\theta}^{\prime}\right)$ denotes the Teichmuller lift of the reduction $\bmod l$ of $\theta_{0}\left(\operatorname{resp} \theta^{\prime}\right)$, and observe that:

- $\theta(\bmod l)=\theta^{\prime}(\bmod l)$. 
- $\theta \theta^{c}=\epsilon_{l}^{-m^{*} n+1-(1-n) w} \tilde{\omega}^{-(w-1)(n-1)}$.

Step 6. Properties of $\operatorname{Ind}_{G_{M}}^{G_{F}} \theta$ and $\operatorname{Ind}_{G_{M}}^{G_{F}} \theta^{\prime}$. We begin by addressing point (4). We define a pairing on $\operatorname{Ind}_{G_{M}}^{G_{F}} \theta$ by the formula

$$
\left\langle\lambda, \lambda^{\prime}\right\rangle=\sum_{\sigma \in \operatorname{Gal}(\bar{M} / M) \backslash \operatorname{Gal}(\bar{M} / F)} \epsilon_{l}(\sigma)^{m^{*} n-1+(1-n) w} \tilde{\omega}(\sigma)^{(w-1)(n-1)} \lambda(\sigma) \lambda^{\prime}(c \sigma),
$$

where $c$ is any complex conjugation. One easily checks that this is well defined and perfect, and that the properties (a) and (b) hold.

We can address point (5) in a similar manner, defining:

$$
\left\langle\lambda, \lambda^{\prime}\right\rangle=\sum_{\sigma \in \operatorname{Gal}(\bar{M} / M) \backslash \operatorname{Gal}(\bar{M} / F)} \epsilon_{l}(\sigma)^{\left(m^{*}-1\right) n} \lambda(\sigma) \lambda^{\prime}(c \sigma)
$$

and checking the required properties.

Next, we address point (6). We will use the following notation: if $S, T$ are multisets of integers, we will write $S \oplus T$ for the 'union with multiplicity' of $S$ and $T$ (so that $\{1\} \oplus\{1\}=\{1,1\}$ ), and $S \otimes T$ for the convolution of $S$ and $T$ (i.e. $\{s+t \mid s \in S, t \in T\}$ with appropriate multiplicities). Finally, for $r: G_{F} \rightarrow \mathrm{GL}_{k}\left(\overline{\mathbb{Z}}_{l}\right)$ a de Rham Galois representation and $v$ a prime above $l$, we will write $\operatorname{HT}_{v}(r)$ for the multiset of Hodge-Tate numbers of $r$ at the place $v$. (Note that $l$ splits completely, so this is well defined.)

Now, supposing $r: \operatorname{Gal}(\bar{F} / F) \rightarrow \mathrm{GL}_{2}\left(\overline{\mathbb{Z}}_{l}\right)$ to be a continuous representation with $\mathrm{HT}_{v}(r)=\left\{-a_{v}, b_{v}-a_{v}\right\}$ for each place $v$ of $F$ above $l$, we can calculate $\mathrm{HT}_{v}\left(\mathrm{Sym}^{n-1} r \otimes \operatorname{Ind}_{G_{M}}^{G_{F}} \theta\right)$ and show it has the required value; see Figure 1

Next we address point (7), in a similar manner. Supposing $r: \operatorname{Gal}(\bar{F} / F) \rightarrow$ $\mathrm{GL}_{2}\left(\overline{\mathbb{Z}}_{l}\right)$ to be a continuous representation with $\operatorname{HT}_{v}(r)=\{0,1\}$ for each place $v$ of $F$ above $l$, we can calculate:

$$
\begin{aligned}
\operatorname{HT}_{v}\left(\operatorname{Sym}^{n-1} r \otimes \operatorname{Ind}_{G_{M}}^{G_{F}} \theta\right) & =\operatorname{HT}_{v}\left(\operatorname{Sym}^{n-1} r\right) \otimes H T_{v}\left(\operatorname{Ind}_{G_{M}}^{G_{F}} \theta\right) \\
& =\{0,1, \ldots, n-1\} \otimes\left\{h_{v, 0}^{\prime}, \ldots, h_{v, m^{*}-1}^{\prime}\right\} \\
& =\{0,1, \ldots, n-1\} \otimes\left\{0, n, 2 n, \ldots,\left(m^{*}-1\right) n\right\} \\
& =\left\{0,1, \ldots, m^{*} n-1\right\} .
\end{aligned}
$$

Next, we address point (2). Let $v \in S_{\mathrm{ss}}$. The assumption that $L^{\prime}$ contains $L$ means that the representations $\rho_{\chi}$ and $\rho_{\chi^{\prime}}$ are both $\mathrm{GL}_{n m^{*}}(\mathcal{O})$-conjugate to direct sums of characters, and the other assumptions on $L^{\prime}$ ensure that these characters are all crystalline. The Hodge-Tate weights of these characters with respect to any embedding $i: L^{\prime} \hookrightarrow \overline{\mathbb{Q}}_{l}$ are determined by the restriction of $i$ to $L$, so we may think of each character as having two Hodge-Tate weights in the obvious way. For both $\rho_{\chi}$ and $\rho_{\chi^{\prime}}$, the set of ordered pairs of Hodge-Tate weights, running over all the characters, is exactly the set of ordered pairs of non-negative integers with sum $n m^{*}-1$ (this follows from the calculations establishing points (6) and (7)). Since two crystalline characters with the same Hodge-Tate weights must differ by an unramified twist, the result follows from Corollary 3.4.4.

Step 7. Establishing the big image/avoid $\zeta_{l}$ properties. All that remains is to prove the big image and avoiding $\zeta_{l}$ properties, that is, point (3). We will just show the stated properties concerning $\operatorname{Ind}_{G_{M}}^{G_{F}} \theta^{\prime}$; the statement for $\operatorname{Ind}_{G_{M}}^{G_{F}} \theta$ then follows since $\theta$ and $\theta^{\prime}$ are congruent. 


$$
\begin{aligned}
& \mathrm{HT}_{v}\left(\operatorname{Sym}^{n-1} r \otimes \operatorname{Ind}_{G_{M}}^{G_{F}} \theta\right) \\
& =\operatorname{HT}_{v}\left(\operatorname{Sym}^{n-1} r\right) \otimes \operatorname{HT}_{v}\left(\operatorname{Ind}_{G_{M}}^{G_{F}} \theta\right) \\
& =\left\{-(n-1) a_{v},-(n-2) a_{v}+\left(b_{v}-a_{v}\right), \ldots,(n-1)\left(b_{v}-a_{v}\right)\right\} \\
& \otimes\left\{h_{v, 0}, \ldots, h_{v, m^{*}-1}\right\} \\
& =\left\{0, b_{v}, \ldots,(n-1) b_{v}\right\} \otimes\left(\left\{-(n-1) a_{v}\right\} \otimes\left\{h_{v, 0}, \ldots, h_{v, m^{*}-1}\right\}\right) \\
& =\left\{0, b_{v}, \ldots,(n-1) b_{v}\right\} \\
& \otimes\left(\left\{0,1,2, \ldots, b_{v}-1\right\} \oplus\left\{n b_{v}, n b_{v}+1, \ldots, n b_{v}+\left(b_{v}-1\right)\right\} \oplus\right. \\
& \left.\cdots \oplus\left\{\left(m^{*}-b_{v}\right) n,\left(m^{*}-b_{v}\right) n+1, \ldots,\left(m^{*}-b_{v}\right) n+b_{v}-1\right\}\right) \\
& =\left\{0, b_{v}, \ldots,(n-1) b_{v}\right\} \otimes\left\{0,1,2, \ldots, b_{v}-1\right\} \\
& \oplus\left\{0, b_{v}, \ldots,(n-1) b_{v}\right\} \otimes\left\{n b_{v}, n b_{v}+1, \ldots, n b_{v}+\left(b_{v}-1\right)\right\} \\
& \oplus \ldots \\
& \oplus\left\{0, b_{v}, \ldots,(n-1) b_{v}\right\} \\
& \otimes\left\{\left(m^{*}-b_{v}\right) n,\left(m^{*}-b_{v}\right) n+1, \ldots,\left(m^{*}-b_{v}\right) n+b_{v}-1\right\} \\
& =\left\{0,1, \ldots, n b_{v}-1\right\} \oplus\left\{n b_{v}, n b_{v}+1, \ldots, 2 n b_{v}-1\right\} \\
& \oplus \cdots \oplus\left\{n\left(m^{*}-b_{v}\right), n\left(m^{*}-b_{v}\right)+1, \ldots, n m^{*}-1\right\} \\
& =\left\{0,1, \ldots, m^{*} n-1\right\}
\end{aligned}
$$

Figure 1. Computation of $\operatorname{HT}_{v}\left(\operatorname{Sym}^{n-1} r \otimes \operatorname{Ind}_{G_{M}}^{G_{F}} \theta\right)$

Let $r$ be a continuous $l$-adic Galois representation with $m^{*}$-big image, such that the following properties hold:

- $r$ is ramified only at primes of $T$ and above $l$, and

- we have $\bar{F}^{\text {ker } \bar{r}}\left(\zeta_{l}\right) \subset F^{(\text {bad) }}$.

We may now apply Lemma 4.1.2. Applying part (2) of that lemma will give that the image of $\left.\left(r \otimes \operatorname{Ind}_{G_{M}}^{G_{F}} \theta^{\prime}\right)\right|_{G_{F\left(\zeta_{l}\right)}}$ is big (the first part of point (3) to be proved) and applying part (1) will give the fact that we avoid $\zeta_{l}$ (the second part of point (3)). All that remains is to check the hypotheses of Lemma 4.1.2

The fact that $M$ is linearly disjoint from $\bar{F}^{\text {ker }} \bar{r}\left(\zeta_{l}\right)$ (common to both parts) comes from the fact that $\bar{F}^{\operatorname{ker} \bar{r}}\left(\zeta_{l}\right) \subset F^{\text {(bad) }}$ and $M$ was chosen to be linearly disjoint from $F^{(\text {bad })}$.

We turn now to the particular hypotheses of the second part. That $r\left(G_{F\left(\zeta_{l}\right)}\right)$ is $m^{*}$-big is by assumption. The properties we require of $\mathfrak{q}$ follow directly from the bullet points established in Step 3, the properties of $r$ just above, and the first and last bullet points (concerning $\theta^{\prime} \theta^{\prime c}$ and $\# \theta^{\prime}\left(I_{\mathfrak{Q}}\right)$ respectively) in the list of properties of $\theta^{\prime}$ given immediately after $\theta^{\prime}$ is introduced in Step 5. The fact that $\left(\bar{\theta}^{\prime}\right)\left(\bar{\theta}^{\prime}\right)^{c}$ can be extended to $G_{F}$ comes from the fact that it is a power of the cyclotomic character.

Finally, we will prove that, when we have applied this lemma, it is in fact possible to strengthen point (2) a little.

Lemma 4.2.5. Suppose that we are in the situation of Lemma 4.2.4, and suppose that $v, L, L^{\prime}, \chi, \chi^{\prime}, \sigma, K, \mathcal{O}$ are as in point (2) of that lemma. Using $\sigma$-standard 
bases, we can consider $\operatorname{Ind}_{G_{L}}^{G_{F_{v}}} \chi$ as a representation $r_{\chi}: G_{F_{v}} \rightarrow \mathrm{GL}_{2}(\mathcal{O})$, and do the same for $r_{\chi^{\prime}}$.

Suppose further that $r, r^{\prime}: G_{F_{v}} \rightarrow \mathrm{GL}_{2}(\mathcal{O})$ are Galois representations and that there is a matrix $A \in \mathrm{GL}_{2}(\mathcal{O})$ such that $r=A r_{\chi} A^{-1}, r^{\prime}=A r_{\chi^{\prime}} A^{-1}$. Let

$$
\begin{aligned}
\rho_{r} & =\left.\left.\left(\operatorname{Sym}^{n-1} r\right)\right|_{G_{L^{\prime}}} \otimes\left(\operatorname{Ind}_{G_{M}}^{G_{F}} \theta\right)\right|_{G_{L^{\prime}}}, \\
\text { and } \rho_{r^{\prime}} & =\left.\left.\left(\operatorname{Sym}^{n-1} r^{\prime}\right)\right|_{G_{L^{\prime}}} \otimes\left(\operatorname{Ind}_{G_{M}}^{G_{F}} \theta^{\prime}\right)\right|_{G_{L^{\prime}}} .
\end{aligned}
$$

We have a given basis of $r$, from which we inherit a basis on $\mathrm{Sym}^{n-1} r$ using Definition 3.3.1. We also have the $\tau$-standard basis on $\operatorname{Ind}_{G_{M}}^{G_{F}} \theta$, and thus we inherit a natural basis on $\rho_{r}$ and can consider it as a representation into $\mathrm{GL}_{n m^{*}}(\mathcal{O})$. The same is true of $\rho_{r^{\prime}}$. 3.3 .5 .

Then $\rho_{r}$ and $\rho_{r^{\prime}}$ are congruent, and moreover $\rho_{r} \sim \rho_{r^{\prime}}$ in the sense of Definition

Proof. The matrix $A$ gives rise to a matrix $A_{n}:=\operatorname{Sym}^{n-1} A$, and (abbreviating Sym $^{n-1} r$ as $r_{n}, \operatorname{Sym}^{n-1} r^{\prime}$ as $r_{n}^{\prime}, \operatorname{Sym}^{n-1} r_{\chi}$ as $r_{\chi, n}$ and $\operatorname{Sym}^{n-1} r_{\chi^{\prime}}$ as $\left.r_{\chi^{\prime}, n}\right)$, $r_{n}=A_{n} r_{\chi, n} A_{n}^{-1}, r_{n}^{\prime}=A_{n} r_{\chi^{\prime}, n} A_{n}^{-1}$.

Then we can define an element $B$ of $\mathrm{GL}_{n m^{*}}(\mathcal{O})$ by putting $B:=A_{n} \otimes \mathrm{id}$, and see that $\rho_{r}=B \rho_{\chi} B^{-1}$ (where $\rho_{\chi}$ is as defined in point (2) of Lemma 4.2.4); similarly $\rho_{r^{\prime}}=B \rho_{\chi^{\prime}} B^{-1}$.

Since point (2) of Lemma 4.2 .4 tells us that $\bar{\rho}_{\chi}=\bar{\rho}_{\chi^{\prime}}$, we have

$$
\bar{\rho}_{r}=\overline{B \rho_{\chi} B^{-1}}=\overline{B \rho_{\chi^{\prime}} B^{-1}}=\bar{\rho}_{r^{\prime}} .
$$

Moreover, since $\rho_{\chi} \sim \rho_{\chi^{\prime}}$ (again from point (2)), we can deduce that

$$
\rho_{r}=B \rho_{\chi} B^{-1} \sim B \rho_{\chi^{\prime}} B^{-1}=\rho_{r^{\prime}}
$$

(since conjugation by $B$ defines a natural isomorphism between the lifting problems for $\bar{\rho}_{\chi}$ and for $\overline{B \rho_{\chi} B^{-1}}$, and hence a natural isomorphism between all the relevant universal lifting rings), as required.

\section{TWISTING AND UNTWISTING}

5.1. Twisting. In this section we establish some basic results about automorphic induction and Galois representations, which are presumably well known but for which we lack a reference. If $K$ is a number field, we say that an automorphic representation $\pi$ of $\mathrm{GL}_{n}\left(\mathbb{A}_{K}\right)$ is regular if $\pi_{v}$ is regular for all $v \mid \infty$, in the sense of Section 7 of BLGHT09. We caution the reader that while "regular algebraic" (which is also defined in Section 7 of [BLGHT09]) implies "regular", the two notions are not equivalent.

Lemma 5.1.1. Suppose that $L / K$ is a cyclic extension of number fields. Let $\kappa$ be a generator of $\operatorname{Gal}(L / K)^{\vee}$. Let $\pi$ be a cuspidal automorphic representation of $\mathrm{GL}_{n}\left(\mathbb{A}_{K}\right)$, and suppose that $\pi \supsetneqq \pi \otimes\left(\kappa^{i} \circ \operatorname{Art}_{K} \circ\right.$ det $)$ for any $1 \leq i \leq[L: K]-1$. Then there is a cuspidal automorphic representation $\Pi$ of $\mathrm{GL}_{n}\left(\mathbb{A}_{L}\right)$ such that for all places $w$ of $L$ lying over a place $v$ of $K$ we have

$$
\operatorname{rec}\left(\Pi_{w}\right)=\left.\operatorname{rec}\left(\pi_{v}\right)\right|_{W_{L_{w}}} .
$$

Proof. By induction on $[L: K]$ we may reduce to the case that $L / K$ is cyclic of prime degree. The result then follows from Theorems 3.4.2 and 3.5.1 of [AC89], together with Lemma VII.2.6 of [HT01] and the main result of Clo82. 
We will write $B C_{L / K}(\pi)$ for $\Pi$.

Lemma 5.1.2. Suppose that $L / K$ is a cyclic extension of number fields of degree $m$. Let $\pi$ be a regular cuspidal automorphic representation of $\mathrm{GL}_{n}\left(\mathbb{A}_{L}\right)$. Let $\sigma$ be a generator of $\operatorname{Gal}(L / K)$. Assume that $\pi ¥ \pi^{\sigma^{i}}$ for any $1 \leq i \leq m-1$. Suppose further that $\operatorname{Ind}_{L_{\infty}}^{K \infty} \pi_{\infty}$ (the local automorphic induction) is regular. Then there is a regular cuspidal automorphic representation $\Pi$ of $\mathrm{GL}_{m n}\left(\mathbb{A}_{K}\right)$ such that for all places $v$ of $K$ we have

$$
\operatorname{rec}\left(\Pi_{v}\right)=\bigoplus_{w \mid v} \operatorname{Ind}_{W_{L_{w}}}^{W_{K_{v}}} \operatorname{rec}\left(\pi_{w}\right)
$$

(the sum being over places $w$ of $L$ dividing $v$ ).

Proof. The case that $m$ is prime follows from Theorem 3.5.1 and Lemma 3.6.4 of AC89, together with Lemma VII.2.6 of [HT01] and the main result of Clo82, (the assumption that $\operatorname{Ind}_{L_{\infty}}^{K_{\infty}} \pi_{\infty}$ is regular is of course equivalent to the statement that $\Pi$ is regular). For the general case we use induction. Suppose that $L \supset L_{2} \supset L_{1} \supset K$ with $L_{2} / L_{1}$ cyclic of prime degree, and suppose that we have found a regular cuspidal automorphic representation $\Pi_{L_{2}}$ of $\mathrm{GL}_{\left[L: L_{2}\right] n}\left(\mathbb{A}_{L_{2}}\right)$ satisfying the analogue of (5.1.1). The result will follow for $L_{1}$ provided we know that $\Pi_{L_{2}} \neq \Pi_{L_{2}}^{\sigma^{\left[L_{1}: K\right] i}}$ for any $1 \leq i \leq\left[L_{2}: L_{1}\right]-1$, but if this fails to hold, then it is easy to see that

$$
\operatorname{Ind}_{L_{\infty}}^{K_{\infty}} \pi_{\infty}=\operatorname{Ind}_{\left(L_{2}\right)_{\infty}}^{K_{\infty}}\left(\operatorname{Ind}_{L_{\infty}}^{\left(L_{2}\right)_{\infty}} \pi_{\infty}\right)=\operatorname{Ind}_{\left(L_{2}\right)_{\infty}}^{K_{\infty}}\left(\Pi_{L_{2}}\right)_{\infty}
$$

cannot be regular, a contradiction.

We will write $\operatorname{Ind}_{L}^{K} \pi$ for $\Pi$.

Let $F$ be a totally real field and let $M$ be an imaginary CM field which is a cyclic Galois extension of $F$ of degree $m$. Fix $\iota: \overline{\mathbb{Q}}_{l} \stackrel{\sim}{\longrightarrow} \mathbb{C}$. Let $\pi$ be an RAESDC automorphic representation of $\mathrm{GL}_{n}\left(\mathbb{A}_{F}\right)$, and let $\chi$ be an algebraic character of $M^{\times} \backslash \mathbb{A}_{M}^{\times}$, chosen so that the Galois representation $\operatorname{Ind}_{G_{M}}^{G_{F}} r_{l, \iota}(\chi)$ is essentially selfdual. Then the Galois representation

$$
r_{l, \iota}(\pi) \otimes \operatorname{Ind}_{G_{M}}^{G_{F}} r_{l, \iota}(\chi): G_{F} \rightarrow \mathrm{GL}_{n m}\left(\overline{\mathbb{Q}}_{l}\right)
$$

is also essentially self-dual. We have the following result.

Proposition 5.1.3. Assume that

$$
\pi_{\infty} \otimes \operatorname{Ind}_{M_{\infty}}^{F_{\infty}} \chi_{\infty}
$$

is regular; equivalently, $r_{l, \iota}(\pi) \otimes \operatorname{Ind}_{G_{M}}^{G_{F}} r_{l, \iota}(\chi)$ is regular. Assume also that if $\kappa$ is a generator of $\operatorname{Gal}(M / F)^{\vee}$, then $\pi \neq \approx \otimes\left(\kappa^{i} \circ \operatorname{Art}_{K} \circ\right.$ det $)$ for any $1 \leq i \leq$ $[M: F]-1$. Then the representation $r_{l, \iota}(\pi) \otimes \operatorname{Ind}_{G_{M}}^{G_{F}} r_{l, \iota}(\chi)$ is automorphic. More precisely, there is an $R A E S D C$ automorphic representation $\Pi$ of $\mathrm{GL}_{n m}\left(\mathbb{A}_{F}\right)$ with $r_{l, \iota}(\Pi) \cong r_{l, \iota}(\pi) \otimes \operatorname{Ind}_{G_{M}}^{G_{F}} r_{l, \iota}(\chi)$. In fact, for every place $v$ of $F$, we have

$$
\operatorname{rec}\left(\Pi_{v}|\cdot|^{(1-m n) / 2}\right)=\operatorname{rec}\left(\pi_{v}|\cdot|^{(1-n) / 2}\right) \otimes\left(\bigoplus_{w \mid v} \operatorname{Ind}_{W_{M_{w}}}^{W_{F_{v}}} \operatorname{rec}\left(\chi_{w}\right)\right)
$$

(the sum being over places $w$ of $M$ dividing $v$ ). 
Proof. It is enough to prove that there is a regular cuspidal automorphic representation $\Pi$ of $\mathrm{GL}_{m n}\left(\mathbb{A}_{F}\right)$ satisfying the final assertion ( $\Pi$ is then algebraic by the conditions at the infinite places, and is automatically essentially self-dual by the strong multiplicity one theorem and the conditions at the finite places, and thus has a Galois representation $r_{l, \iota}(\Pi)$ associated to it, which satisfies the required condition by the Tchebotarev density theorem).

By Lemma 5.1.1 and Lemma 5.1.2 we have a regular cuspidal automorphic representation

$$
\Pi:=\left(\operatorname{Ind}_{M}^{F}\left(B C_{M / F}\left(\pi|\cdot|^{(1-n) / 2}\right) \otimes \chi\right)\right)|\cdot|^{(m n-1) / 2}
$$

(note that $B C_{M / F}\left(\pi|\cdot|^{(1-n) / 2}\right) \otimes \chi$ satisfies the hypotheses of Lemma 5.1 .2 by the assumption that $\pi_{\infty} \otimes \operatorname{Ind}_{M_{\infty}}^{F_{\infty}} \chi_{\infty}$ is regular). By definition this choice of $\Pi$ satisfies

$$
\operatorname{rec}\left(\Pi_{v}|\cdot|^{(1-m n) / 2}\right)=\bigoplus_{w \mid v} \operatorname{Ind}_{W_{M_{w}}}^{W_{F_{v}}}\left(\left.\operatorname{rec}\left(\pi_{v}|\cdot|^{(1-n) / 2}\right)\right|_{W_{M_{w}}} \otimes \operatorname{rec}\left(\chi_{w}\right)\right)
$$

for each place $v$ of $F$, and the result follows.

5.2. Untwisting. In this section we explain a kind of converse to Proposition 5.1.3. following an idea of Harris ( Har09, although our exposition is extremely similar to that found in the proof of Theorems 7.5 and 7.6 of BLGHT09]).

Suppose that $F$ is a totally real field and that $M$ is an imaginary CM field which is a cyclic extension of $F$ of degree $m$. Suppose that $\theta$ is an algebraic character of $M^{\times} \backslash \mathbb{A}_{M}^{\times}$and that $\Pi$ is a RAESDC representation of $\mathrm{GL}_{m n}\left(\mathbb{A}_{F}\right)$ for some $n$. Let $\iota: \overline{\mathbb{Q}}_{l} \stackrel{\sim}{\longrightarrow} \mathbb{C}$.

Proposition 5.2.1. Assume that there is a continuous irreducible representation $r: G_{F} \rightarrow \mathrm{GL}_{n}\left(\overline{\mathbb{Q}}_{l}\right)$ such that $\left.r\right|_{G_{M}}$ is irreducible and

$$
r_{l, \iota}(\Pi) \cong r \otimes \operatorname{Ind}_{G_{M}}^{G_{F}} r_{l, \iota}(\theta) .
$$

Then $r$ is automorphic.

Proof. Let $\sigma$ denote a generator of $\operatorname{Gal}(M / F)$, and $\kappa$ a generator of $\operatorname{Gal}(M / F)^{\vee}$. Then we have

$$
\begin{aligned}
r_{l, \iota}\left(\Pi \otimes\left(\kappa \circ \operatorname{Art}_{F} \circ \text { det }\right)\right) & =r_{l, \iota}(\Pi) \otimes r_{l, \iota}\left(\kappa \circ \operatorname{Art}_{F}\right) \\
& \cong r \otimes\left(r_{l, \iota}\left(\kappa \circ \operatorname{Art}_{F}\right) \otimes \operatorname{Ind}_{G_{M}}^{G_{F}} r_{l, \iota}(\theta)\right) \\
& \cong r \otimes \operatorname{Ind}_{G_{M}}^{G_{F}}\left(\left.r_{l, \iota}\left(\kappa \circ \operatorname{Art}_{F}\right)\right|_{G_{M}} \otimes r_{l, \iota}(\theta)\right) \\
& \cong r \otimes \operatorname{Ind}_{G_{M}}^{G_{F}} r_{l, \iota}(\theta) \\
& \cong r_{l, \iota}(\Pi),
\end{aligned}
$$

so that $\Pi \otimes\left(\kappa \circ \operatorname{Art}_{F} \circ\right.$ det $) \cong \Pi$.

We claim that for each intermediate field $M \supset N \supset F$ there is a regular cuspidal automorphic representation $\Pi_{N}$ of $\mathrm{GL}_{n[M: N]}$ such that

$$
\Pi_{N} \otimes\left(\kappa \circ \operatorname{Art}_{N} \circ \operatorname{det}\right) \cong \Pi_{N}
$$

and $B C_{N / F}(\Pi)$ is equivalent to

$$
\Pi_{N} \boxplus \Pi_{N}^{\sigma} \boxplus \cdots \boxplus \Pi_{N}^{\sigma^{[N: F]-1}}
$$

in the sense that for all places $v$ of $N$, the base change from $F_{\left.v\right|_{F}}$ to $N_{v}$ of $\Pi_{\left.v\right|_{F}}$ is

$$
\Pi_{N, v} \boxplus \Pi_{N, v}^{\sigma} \boxplus \cdots \boxplus \Pi_{N, v}^{\sigma^{[N: F]-1}} .
$$


We prove this claim by induction on $[N: F]$. Suppose that $M \supset M_{2} \supset M_{1} \supset F$ with $M_{2} / M_{1}$ cyclic of prime degree, and that we have already proved the result for $M_{1}$. Since

$$
\Pi_{M_{1}} \otimes\left(\kappa \circ \operatorname{Art}_{M_{1}} \circ \operatorname{det}\right) \cong \Pi_{M_{1}},
$$

we see from Theorems 3.4.2 and 3.5.1 of AC89 (together with Lemma VII.2.6 of [HT01] and the main result of [Clo82]) that there is a cuspidal automorphic representation $\Pi_{M_{2}}$ of $\mathrm{GL}_{n\left[M: M_{2}\right]}$ such that $B C_{M_{2} / F}(\Pi)$ is equivalent to

$$
\Pi_{M_{2}} \boxplus \Pi_{M_{2}}^{\sigma} \boxplus \cdots \boxplus \Pi_{M_{2}}^{\sigma^{\left[M_{2}: F\right]-1}} .
$$

Since $\Pi$ is regular, $\Pi_{M_{2}}$ is regular. The representation $\Pi_{M_{2}} \otimes\left(\kappa \circ \operatorname{Art}_{M_{2}} \circ\right.$ det $)$ satisfies the same properties (because $\Pi \otimes\left(\kappa \circ \operatorname{Art}_{F} \circ\right.$ det $\left.) \cong \Pi\right)$, so we see (by strong multiplicity one for isobaric representations) that we must have

$$
\Pi_{M_{2}} \otimes\left(\kappa \circ \operatorname{Art}_{M_{2}} \circ \operatorname{det}\right) \cong \Pi_{M_{2}}^{\sigma^{i}}
$$

for some $0 \leq i \leq\left[M: M_{2}\right]-1$. If $i>0$, then

$$
\Pi_{M_{2}} \boxplus \Pi_{M_{2}}^{\sigma} \boxplus \cdots \boxplus \Pi_{M_{2}}^{\sigma^{\left[M_{2}: F\right]-1}}
$$

cannot be regular (note that of course $\kappa$ is a character of finite order), a contradiction, so in fact we must have $i=0$. Thus

$$
\Pi_{M_{2}} \otimes\left(\kappa \circ \operatorname{Art}_{M_{2}} \circ \text { det }\right) \cong \Pi_{M_{2}},
$$

and the claim follows.

Let $\pi:=\Pi_{M}$. Note that the representations $\pi^{\sigma^{i}}$ for $0 \leq i \leq m-1$ are pairwise non-isomorphic (because $\Pi$ is regular). Note also that $\pi \otimes|\operatorname{det}|^{(n-n m) / 2}$ is regular algebraic (again, because $\Pi$ is regular algebraic).

Since $\Pi$ is RAESDC, there is an algebraic character $\chi$ of $F^{\times} \backslash \mathbb{A}_{F}^{\times}$such that $\Pi^{\vee} \cong \Pi \otimes$ ( $\chi \circ$ det). It follows (by strong multiplicity one for isobaric representations) that for some $0 \leq i \leq m-1$ we have

$$
\pi^{\vee} \cong \pi^{\sigma^{i}} \otimes\left(\chi \circ N_{M / F} \circ \operatorname{det}\right)
$$

Then we have

$$
\begin{aligned}
\pi & \cong\left(\pi^{\vee}\right)^{\vee} \\
& \cong\left(\pi^{\sigma^{i}} \otimes\left(\chi \circ N_{M / F} \circ \text { det }\right)\right)^{\vee} \\
& \cong\left(\pi^{\vee}\right)^{\sigma^{i}} \otimes\left(\chi^{-1} \circ N_{M / F} \circ \text { det }\right) \\
& \cong\left(\pi^{\sigma^{i}} \otimes\left(\chi \circ N_{M / F} \circ \text { det }\right)\right)^{\sigma^{i}} \otimes\left(\chi^{-1} \circ N_{M / F} \circ \operatorname{det}\right) \\
& \cong \pi^{\sigma^{2 i}}
\end{aligned}
$$

so that either $i=0$ or $i=m / 2$. We wish to rule out the former possibility. Assume for the sake of contradiction that

$$
\pi^{\vee} \cong \pi \otimes\left(\chi \circ N_{M / F} \circ \text { det }\right) .
$$

Since $F$ is totally real, there is an integer $w$ such that $\chi|\cdot|^{-w}$ has finite image. Then $\pi|\operatorname{det}|^{w / 2}$ has unitary central character, so is itself unitary. Since $\pi \otimes|\operatorname{det}|^{(n-n m) / 2}$ 
is regular algebraic, we see that for places $v \mid \infty$ of $M$ the conditions of Lemma 7.1 of [BLGHT09] are satisfied for $\pi_{v}|\operatorname{det}|_{v}^{w / 2}$, so that

$$
\begin{aligned}
\pi_{v} \boxplus \pi_{v}^{c} & \cong \pi_{v} \boxplus\left(\left(\pi_{v} \otimes|\operatorname{det}|^{w / 2}\right)^{c} \otimes\left(|\cdot|^{-w / 2} \circ \operatorname{det}\right)\right) \\
& \cong \pi_{v} \boxplus\left(\left(\pi_{v} \otimes|\operatorname{det}|^{w / 2}\right)^{\vee} \otimes\left(|\cdot|^{-w / 2} \circ \operatorname{det}\right)\right) \\
& \cong \pi_{v} \boxplus\left(\pi_{v}^{\vee} \otimes\left(|\cdot|^{-w} \circ \operatorname{det}\right)\right) \\
& \cong \pi_{v} \boxplus\left(\pi_{v} \otimes\left(\chi|\cdot|^{-w} \circ N_{M / F} \circ \operatorname{det}\right)\right),
\end{aligned}
$$

which contradicts the regularity of $\Pi_{\left.v\right|_{F}}$. Thus we have $i=m / 2$, so that

$$
\pi^{\vee} \cong \pi^{c} \otimes\left(\chi \circ N_{M / F} \circ \text { det }\right) \text {. }
$$

Thus $\pi \otimes|\operatorname{det}|^{(n-n m) / 2}$ is a RAECSDC representation, so that we have a Galois representation $r_{l, \iota}\left(\pi \otimes|\operatorname{det}|^{(n-n m) / 2}\right)$. The condition that $B C_{M / F}(\Pi)$ is equivalent to

$$
\pi \boxplus \pi^{\sigma} \boxplus \cdots \boxplus \pi^{\sigma^{m-1}}
$$

translates to the fact that

$$
\left.r_{l, L}(\Pi)\right|_{G_{M}} \cong r_{l, L}\left(\pi \otimes|\operatorname{det}|^{(n-n m) / 2}\right) \oplus \cdots \oplus r_{l, L}\left(\pi \otimes|\operatorname{det}|^{(n-n m) / 2}\right)^{\sigma^{m-1}} .
$$

By hypothesis, we also have

$$
\left.r_{l, \iota}(\Pi)\right|_{G_{M}} \cong\left(\left.r\right|_{G_{M}} \otimes r_{l, \iota}(\theta)\right) \oplus \cdots \oplus\left(\left.r\right|_{G_{M}} \otimes r_{l, \iota}(\theta)^{\sigma^{m-1}}\right) .
$$

Since $\left.r\right|_{G_{M}}$ is irreducible, there must be an $i$ such that

$$
\left.r\right|_{G_{M}} \cong r_{l, \iota}\left(\pi \otimes|\operatorname{det}|^{(n-n m) / 2}\right) \otimes r_{l, \iota}(\theta)^{\sigma^{-i}},
$$

so that $\left.r\right|_{G_{M}}$ is automorphic. The result now follows from Lemmas 1.4 and 1.5 of BLGHT09.

\section{Potential automorphy in weight 0}

6.1. In our final arguments, we will need to rely on certain potential automorphy results for symmetric powers of Galois representations with Hodge-Tate numbers $\{0,1\}$ at every place. The fact that such results are immediately available given the techniques in the literature is well known to the experts, but because we were unable to locate a reference which states the precise results we will need, we will give very brief derivations of them here. We hope that providing a written reference for these results may prove useful to other authors.

Lemma 6.1.1. Suppose that $l>2(n-1) m+1$ is a prime, that $k$ is an algebraic extension of $\mathbb{F}_{l}$, that $k^{\prime} \subseteq k$ is a finite field and that $H \subset \mathrm{GL}_{n}(k)$. Suppose that

$$
k^{\times} \operatorname{Sym}^{n-1} \mathrm{GL}_{2}\left(k^{\prime}\right) \supseteq H \supseteq \operatorname{Sym}^{n-1} \mathrm{SL}_{2}\left(k^{\prime}\right) .
$$

Then $H$ is m-big.

Proof. This may be deduced from Lemma 7.3 of [BLGHT09] as Corollary 2.5.4 of CHT08 is deduced from Lemma 2.5.2 of loc. cit.

Lemma 6.1.2. Suppose that $m$ is a positive integer, that $k$ is an algebraic extension of $\mathbb{F}_{l}$, that $k^{\prime} \subseteq k$ is a finite field and that $F$ is a totally real field. Suppose that $l$ is a prime such that $\left[F\left(\zeta_{l}\right): F\right]>2 m$, and that $\bar{r}: \operatorname{Gal}(\bar{F} / F) \rightarrow \mathrm{GL}_{2}(k)$ has

$$
k^{\times} \mathrm{GL}_{2}\left(k^{\prime}\right) \supseteq \bar{r}\left(G_{F}\right) \supseteq \mathrm{SL}_{2}\left(k^{\prime}\right) .
$$


Then, for any $n,\left[\bar{F}^{\mathrm{kerad} \operatorname{Sym}^{n-1} \bar{r}}\left(\zeta_{l}\right): \bar{F}^{\text {ker ad Sym }}{ }^{n-1} \bar{r}\right]>m$. In particular, the conclusion holds if $l$ is unramified in $F$ and $l>2 m+1$.

Proof. We have

$$
\mathrm{PSL}_{2}\left(k^{\prime}\right) \subset\left(\operatorname{ad~Sym}^{n-1} \bar{r}\right)\left(G_{F}\right) \subset \mathrm{PGL}_{2}\left(k^{\prime}\right)
$$

$\mathrm{PGL}_{2}\left(k^{\prime}\right) / \mathrm{PSL}_{2}\left(k^{\prime}\right)$ has order 2 , and $\mathrm{PSL}_{2}\left(k^{\prime}\right)$ is simple. Thus the intersection of $\bar{F}^{\text {ad Sym }^{n-1} \bar{r}}$ and $F\left(\zeta_{l}\right)$ has degree at most 2 over $F$. Since $\left[F\left(\zeta_{l}\right): F\right]>2 m$, the result follows (for the final part, note that if $l$ is unramified in $F$, then $\left[F\left(\zeta_{l}\right): F\right]=$ $l-1)$.

Proposition 6.1.3. Let $F$ be a totally real field, $F^{\text {(avoid) }}$ a finite extension of $F$, $\mathcal{L}$ a finite set of primes of $F, n$ a positive integer, and $l>4(n-1)+1$ a prime which is unramified in F. Suppose that

$$
r: G_{F} \rightarrow \mathrm{GL}_{2}\left(\mathbb{Z}_{l}\right)
$$

is a continuous representation which is unramified at all but finitely many primes and enjoys the following properties:

(1) $\operatorname{det} r=\epsilon_{l}^{-1}$.

(2) $\bar{r}\left(G_{F}\right) \supset \mathrm{SL}_{2}\left(\mathbb{F}_{l}\right)$.

(3) For each prime $v \mid l$ of $F,\left.r\right|_{G_{F_{v}}}$ is crystalline for all $\tau \in \operatorname{Hom}\left(F, \overline{\mathbb{Q}}_{l}\right)$, and we have

$$
\operatorname{dim}_{\overline{\mathbb{Q}}_{l}} \operatorname{gr}^{i}\left(r \otimes_{\tau, F_{v}} \mathrm{~B}_{\mathrm{dR}}\right)^{G_{F_{v}}}= \begin{cases}1 & i=0,1, \\ 0 & \text { (otherwise) } .\end{cases}
$$

(4) $\mathcal{L}$ does not contain primes above $l$, and $r$ is unramified at places in $\mathcal{L}$.

Then there is a Galois totally real extension $F^{\prime \prime}$ of $F$, linearly disjoint from $F^{\text {(avoid) }}$ over $F$ such that $\left.\left(\mathrm{Sym}^{n-1} r\right)\right|_{G_{F^{\prime \prime}}}$ is automorphic of weight 0 and level prime to $\mathrm{l}$, and no prime of $\mathcal{L} \cup\{v \mid v$ a prime of $F, v \mid l\}$ ramifies in $F^{\prime \prime}$.

Proof. We will deduce this result from Theorem 7.5 of [BLGHT09] in the same way that Theorem 3.2 of [HSBT10] is deduced from Theorem 3.1 of [HSBT10, following the proof of Theorem 3.2 of HSBT10 very closely. Our argument is in fact simpler, because we need no longer maintain a Steinberg place, and so when we apply the theorem of Moret-Bailly (Proposition 2.1 of [HSBT10]), we do not impose any local condition at auxiliary primes $v_{q}, v_{q^{\prime}}$, unlike in [HSBT10. Thus all arguments earlier on in the proof concerning these primes become unnecessary.

In particular, we copy the argument up to the application of Proposition 2.1 of HSBT10] almost verbatim, with only the following changes:

- The character $\epsilon_{l} \operatorname{det} r$ is trivial, so the field $F_{1}$ is simply $F$, and in particular, $F_{1}$ is linearly disjoint from $F^{(\text {avoid })}$ over $F$.

- Rather than choosing $l^{\prime}>C(n), n$, we take $l^{\prime}>4(n-1)+1, l^{\prime}>5$ and $l^{\prime} \notin \mathcal{L}$.

- When we choose $E_{1}$ in the application of Proposition 2.1 of HSBT10, we choose it to have good reduction at primes of $\mathcal{L}$.

- As mentioned above, we no longer impose any local condition at the primes $v_{q}, v_{q^{\prime}}$ since we no longer need the conclusion, after the application of Proposition 2.1 of [HSBT10], that $E$ has split multiplicative reduction at auxiliary primes $v_{q}, v_{q^{\prime}}$. 
- In the application of Proposition 2.1 of [HSBT10, we may also assume that the field $F^{\prime}$ is linearly disjoint from $F^{\text {(avoid) }}$ (this is easy, as Proposition 2.1 of [HSBT10] allows us to avoid any fixed field). We also impose a local condition to ask that $F$ not ramify at primes of $\mathcal{L}$; we must then check that we can find some elliptic curve whose mod $l l^{\prime}$ cohomology agrees with $H^{1}\left(E_{1} \times \overline{\mathbb{F}}, \mathbb{Z} / l^{\prime} \mathbb{Z}\right) \times \bar{r}$ when restricted to inertia at primes of $\mathcal{L}$. $E_{1}$ itself fills this role, all the representations involved being trivial in this case.

As in HSBT10, the result of all this is the construction of an elliptic curve $E$ over a finite Galois extension $F^{\prime}$ of $F$, which together have the following properties:

- $F^{\prime}$ is linearly disjoint from $F^{\text {(avoid) }} \bar{F}^{\operatorname{ker}\left(\bar{r} \times \bar{r}_{1}\right)}$, where $\bar{r}_{1}$ is the Galois representation $H^{1}\left(E_{1} \times \bar{F}, \mathbb{Z} / l^{\prime} \mathbb{Z}\right)$ attached to a certain elliptic curve $E_{1}$ chosen earlier in the portion of the proof we followed from [HSBT10, as mentioned above.

- In particular, since $E_{1}$ was chosen such that $G_{F} \rightarrow$ Aut $H^{1}\left(E_{1} \times \bar{F}, \mathbb{Z} / l^{\prime} \mathbb{Z}\right)$, we also have $G_{F^{\prime}} \rightarrow$ Aut $H^{1}\left(E_{1} \times \bar{F}, \mathbb{Z} / l^{\prime} \mathbb{Z}\right)$.

- $F^{\prime}$ is totally real.

- All primes above $l l^{\prime}$ and the primes of $\mathcal{L}$ are unramified in $F^{\prime}$.

- $E$ has good reduction at all places above $l l^{\prime}$ and the primes of $\mathcal{L}$.

- $\left.H^{1}(E \times \bar{F}, \mathbb{Z} / l \mathbb{Z}) \cong r\right|_{G_{F^{\prime}}}$

- $E$ has good ordinary reduction at $l^{\prime}$ (note that $l^{\prime}$ is unramified in $F$, that $E$ has good reduction at $l^{\prime}$, and that its $l^{\prime}$ torsion is isomorphic to the $l^{\prime}$ torsion of $E_{1}$, which was chosen to be ordinary at $l^{\prime}$ ).

We then apply Theorem 7.5 of BLGHT09 5 to the Galois representation Sym $^{n-1} H^{1}\left(E \times \bar{F}, \mathbb{Z}_{l^{\prime}}\right)$, which we will call $r_{n}^{\prime}$ for brevity. Let us check the conditions of this theorem in turn. We have two unnumbered conditions: first that $r_{n}^{\prime}$ is almost everywhere unramified (which is obvious, as it comes from geometry), and second that there is a perfect, Galois equivariant, pairing on $r_{n}^{\prime}$ towards $\mathbb{Z}_{l}(\mu)$, for some character $\mu$ (such a pairing on $H^{1}\left(E \times \overline{\mathbb{F}}, \mathbb{Z}_{l^{\prime}}\right)$ is furnished by Poincaré duality, going towards $\mathbb{Z}_{l}(-1)$; thus we get such a pairing on $r_{n}^{\prime}$ with $\left.\mu=\epsilon_{l}^{1-n}\right)$. Now we address the numbered conditions:

1: The sign of $\mu$ on complex conjugations agrees with the parity of the pairing. Poincaré duality on $H^{1}\left(E \times \overline{\mathbb{F}}, \mathbb{Z}_{l^{\prime}}\right)$ will be an alternating pairing, so the pairing on $r_{n}^{\prime}$ will satisfy $\langle u, v\rangle=(-1)^{1-n}\langle v, u\rangle$ and $\mu=\epsilon_{l}^{1-n}$ is $(-1)^{1-n}$ on complex conjugations.

2: We have $\left[\bar{F}^{\text {ker ad } \bar{r}_{n}^{\prime}}\left(\zeta_{l}\right): \bar{F}^{\text {ker ad } \bar{r}_{n}^{\prime}}\right]>2$. We can use the fact that the Galois representation on the $l^{\prime}$ torsion of $E$ is surjective (since it agrees with the action on the $l^{\prime}$ torsion of $E_{1}$, and using point (A1) above), and Lemma 6.1 .2 (using the fact that $l^{\prime}>5$ ).

3: We have that $\bar{r}_{n}^{\prime}\left(G_{F\left(\zeta_{l}\right)}\right)$ is 2-big. Again we use the fact that the Galois representation on the $l^{\prime}$ torsion of $E$ is surjective, this time together with the simplicity of $\mathrm{PSL}_{2}(k)$, and Lemma 6.1.1 (using $l^{\prime}>4(n-1)+1$ ).

4: $r_{n}^{\prime}$ is ordinary of regular weight. This is immediate given point (A2) above.

\footnotetext{
${ }^{5}$ In fact, we need a slight extension incorporating a set of primes $\mathcal{L}$ where we do not want our extension to ramify, and a fixed field from which we want our extension to be linearly disjoint. Adding this is a routine modification of the proof of Theorem 6.3 of [BLGHT09], in the same spirit as the modifications above of the arguments of HSBT10.
} 
We immediately deduce that there exists an extension $F^{\prime \prime} / F^{\prime}$, with $\operatorname{Sym}^{n-1} H^{1}(E \times$ $\left.\overline{\mathbb{F}}, \mathbb{Z}_{l^{\prime}}\right)\left.\right|_{G_{F^{\prime \prime}}}$ automorphic of weight 0 and level prime to $l$, and such that

- $F^{\prime \prime} / F$ is Galois.

- Primes above $l$ and $l^{\prime}$ are unramified in $F^{\prime \prime}$, as are the primes of $\mathcal{L}$.

- $F^{\prime \prime}$ is linearly disjoint from $F^{\text {(avoid) }} F^{\prime} \bar{F}^{\text {ker ad } \bar{r}} \bar{F}^{\text {ker ad } \bar{r}_{n}^{\prime}}$ over $F^{\prime}$ (and hence linearly disjoint from $F^{\text {(avoid) }} \bar{F}^{\text {ker ad } \bar{r}}$ over $F$ ).

We now apply Theorem 5.2 of Gue09 to the Galois representation $\left.\operatorname{Sym}^{n-1} r\right|_{G_{F^{\prime \prime}}}$, which we call $r_{n}$ for brevity. Let us check the conditions of this theorem in turn:

1: $\left.r_{n}\right|_{G_{F^{\prime \prime}}}$ is essentially self-dual. $r$ acquires a perfect symplectic pairing with cyclotomic multiplier from the determinant; from this, $\left.r_{n}\right|_{G_{F^{\prime \prime}}}$ inherits a perfect pairing with the desired properties.

2: $\left.r_{n}\right|_{G_{F^{\prime \prime}}}$ is almost everywhere unramified. This is trivial, since we assume $r$ has this property.

3: $\left.r_{n}\right|_{G_{F^{\prime \prime}}}$ is crystalline at each place above $l$. This too is trivial, since we assume $r$ has this property (condition (3) of the theorem being proved).

4: $\left.r_{n}\right|_{G_{F^{\prime \prime}}}$ is regular with Hodge-Tate weights lying in the Fontaine-Laffaille range. It follows from condition (3) of the theorem being proved that the Hodge-Tate weights of $\left.r_{n}\right|_{G_{F^{\prime \prime}}}$ are $\{0,1, \ldots, n-1\}$; this suffices, as $l>n$.

5: $\bar{F}^{\text {ker ad } \bar{r}_{n}}$ does not contain $\zeta_{l}$. This follows by condition (2) of the theorem being proved, the fact $l>3$, and point (A3) above, using Lemma 6.1.2.

6: $\bar{r}_{n}\left(G_{F^{\prime \prime}\left(\zeta_{l}\right)}\right)$ is big. This is true by condition (2) of the theorem being proved, the simplicity of $P S L_{2}\left(\mathbb{F}_{l}\right)$, and point (A3) above, as we can see by applying Lemma 6.1.1.

7: $\bar{r}_{n}$ is automorphic, with the right weight. We know that $r$ and $H^{1}(E \times$ $\left.\overline{\mathbb{F}}, \mathbb{Z}_{l}\right)$ are congruent, and manifestly $\operatorname{Sym}^{n-1} H^{1}\left(E \times \overline{\mathbb{F}}, \mathbb{Z}_{l}\right)$ has Hodge-Tate weights $\{0,1, \ldots, n-1\}$, which is indeed the required weight as we saw in verifying hypothesis 4 above.

We conclude that $\operatorname{Sym}^{n-1} r$ is automorphic over $F^{\prime \prime}$ of weight 0 and level prime to $l$, as required.

\section{HilberT MOdUlar FORMS}

7.1. Let $\pi$ be a regular algebraic cuspidal automorphic representation of $\mathrm{GL}_{2}\left(\mathbb{A}_{F}\right)$, where $F$ is a totally real field. Assume that $\pi$ is not of CM type. Let the weight of $\pi$ be $\lambda \in\left(\mathbb{Z}_{+}^{2}\right)^{\operatorname{Hom}(F, \mathbb{C})}$. Let $m^{*}$ be the least common multiple of 2 and the values $\lambda_{v, 1}-\lambda_{v, 2}+1$. Let $n>1$ be a positive integer. Choose a prime $l>5$, and fix an isomorphism $\iota: \overline{\mathbb{Q}}_{l} \stackrel{\sim}{\longrightarrow} \mathbb{C}$. We choose $l$ so that:

- $l$ splits completely in $F$.

- $\pi_{v}$ is unramified for all $v \mid l$.

- The residual representation $\bar{r}_{l, L}(\pi): G_{F} \rightarrow \mathrm{GL}_{2}\left(\overline{\mathbb{F}}_{l}\right)$ has a large image, in the sense that there are finite fields $\mathbb{F}_{l} \subset k \subset k^{\prime}$ with

$$
\mathrm{SL}_{2}(k) \subset \bar{r}_{l, L}(\pi)\left(G_{F}\right) \subset k^{\prime \times} \mathrm{GL}_{2}(k) .
$$

(Note that this is automatically the case for all sufficiently large $l$ by Proposition 0.1 of [Dim05].)

- $l>2(n-1) m^{*}+1$.

Note that, as a consequence of points (B1) and (B2), the simplicity of $\mathrm{PSL}_{2}\left(\mathbb{F}_{l}\right)$, and Lemmas 6.1.1 and 6.1.2, it follows that: 
- The image of $\operatorname{Sym}^{n-1} r_{l, \iota}(\pi)$ is $m^{*}$-big.

- $\left[\bar{F}^{\text {ker ad } \operatorname{Sym}^{n-1} \bar{r}_{l, \iota}(\pi)}\left(\zeta_{l}\right): \bar{F}^{\text {ker ad } \operatorname{Sym}^{n-1} \bar{r}_{l, L}(\pi)}\right]>m^{*}$.

Choose a solvable extension $F^{\prime} / F$ of totally real fields such that

- $l$ splits completely in $F^{\prime}$.

- $F^{\prime}$ is linearly disjoint from $\bar{F}^{\text {ker } \bar{r}_{l, L}(\pi)}$ over $F$.

- At each place $w$ of $F^{\prime}, \pi_{F^{\prime}, w}$ is either unramified or an unramified twist of the Steinberg representation (here we let $\pi_{F^{\prime}}$ denote the base change of $\pi$ to $\left.F^{\prime}\right)$.

- $\left[F^{\prime}: \mathbb{Q}\right]$ is even.

That such an extension exists follows exactly as in the proof of Theorem 3.5.5 of Kis09]. After a further quadratic base change if necessary, we may also assume that

- $\pi_{F^{\prime}}$ is ramified at an even number of places.

Proposition 7.1.1. There is a cuspidal algebraic automorphic representation $\pi^{\prime}$ of $\mathrm{GL}_{2}\left(\mathbb{A}_{F^{\prime}}\right)$ such that

(1) $\pi^{\prime}$ has weight 0 .

(2) $\left.\bar{r}_{l, \iota}\left(\pi^{\prime}\right) \cong \bar{r}_{l, \iota}(\pi)\right|_{G_{F^{\prime}}}$.

(3) if $w \nmid l$ is a place of $F^{\prime}$, then $\pi_{w}^{\prime}$ is ramified if and only if $\pi_{F^{\prime}, w}$ is, in which case it is also an unramified twist of the Steinberg representation.

(4) $\left.r_{l, L}\left(\pi^{\prime}\right)\right|_{G_{F^{\prime}}}$ is potentially Barsotti-Tate for all $v \mid l$, and is ordinary if and only if $\left.r_{l, \iota}(\pi)\right|_{G_{F_{v}^{\prime}}}$ is ordinary.

Proof. Choose a quaternion algebra $B$ with centre $F^{\prime}$ which is ramified at precisely the infinite places and the set $\Sigma$ of finite places at which $\pi_{F^{\prime}}$ is ramified. We will use Lemma 3.1.4 of Kis09] and the Jacquet-Langlands correspondence to find $\pi^{\prime}$. Let $v \in \Sigma$ and let $\rho$ be an irreducible representation of $B_{v}^{\times}$corresponding to the irreducible admissible representation $\mathrm{JL}(\rho)$ of $\mathrm{GL}_{2}\left(F_{v}^{\prime}\right)$ under the JacquetLanglands correspondence. We recall that $\rho^{\mathcal{O}_{B, v}^{\times}}$is non-zero if and only if $\operatorname{JL}(\rho)$ is an unramified twist of the Steinberg representation. We now introduce $l$-adic automorphic forms on $B^{\times}$. Let $K$ be a finite extension of $\mathbb{Q}_{l}$ inside $\overline{\mathbb{Q}}_{l}$ with ring of integers $\mathcal{O}$ and residue field $k$, and assume that $K$ contains the images of all embeddings $F^{\prime} \hookrightarrow \overline{\mathbb{Q}}_{l}$. Fix a maximal order $\mathcal{O}_{B}$ in $B$ and for each finite place $v \notin \Sigma$ of $F^{\prime}$ fix an isomorphism $i_{v}: \mathcal{O}_{B, v} \stackrel{\sim}{\longrightarrow} M_{2}\left(\mathcal{O}_{F_{v}^{\prime}}\right)$. Since $l$ splits completely in $F^{\prime}$, we can and do identify embeddings $F^{\prime} \hookrightarrow \overline{\mathbb{Q}}_{l}$ with primes of $F^{\prime}$ dividing $l$. For each place $v \mid l$ we let $\iota v$ denote the real place of $F^{\prime}$ corresponding to the embedding $\iota \circ v$. Similarly, if $\sigma: F^{\prime} \hookrightarrow \mathbb{R}$ is an embedding we let $\iota^{-1} \sigma$ denote the corresponding place of $F^{\prime}$ dividing $l$.

For each $\lambda^{\prime} \in\left(\mathbb{Z}_{+}^{2}\right)^{\operatorname{Hom}\left(F^{\prime}, \overline{\mathbb{Q}}_{l}\right)}$ and $v \mid l$ consider the algebraic representation

$$
W_{\lambda_{v}^{\prime}}:=\operatorname{Sym}^{\lambda_{v, 1}^{\prime}-\lambda_{v, 2}^{\prime}} \mathcal{O}^{2} \otimes_{\mathcal{O}}(\operatorname{det})^{\lambda_{v, 2}^{\prime}}
$$

of $\mathrm{GL}_{2}(\mathcal{O})$. We consider it as a representation of $\mathcal{O}_{B, v}^{\times}$via $\mathcal{O}_{B, v}^{\times} \stackrel{i_{v}}{\longrightarrow} \mathrm{GL}_{2}\left(\mathcal{O}_{F_{v}^{\prime}}\right) \stackrel{v}{\hookrightarrow}$ $\mathrm{GL}_{2}(\mathcal{O})$. Let $W_{\lambda^{\prime}}=\bigotimes_{v \mid l} W_{\lambda_{v}^{\prime}}$ considered as a representation of $\mathrm{GL}_{2}\left(\mathcal{O}_{F^{\prime}, l}\right)$. For each $v \mid l$ let $\tau_{v}$ denote a smooth representation of $\mathrm{GL}_{2}\left(\mathcal{O}_{F_{v}^{\prime}}\right)$ on a finite free $\mathcal{O}$-module $W_{\tau_{v}}$. Let $\tau$ denote the representation $\bigotimes_{v \mid l} \tau_{v}$ of $\mathrm{GL}_{2}\left(\mathcal{O}_{F^{\prime}, l}\right)$ on $W_{\tau}:=\bigotimes_{v \mid l} W_{\tau_{v}}$. We let $W_{\lambda^{\prime}, \tau}=W_{\lambda^{\prime}} \otimes_{\mathcal{O}} W_{\tau}$. Suppose that $\psi^{\prime}:\left(F^{\prime}\right)^{\times} \backslash\left(\mathbb{A}_{F^{\prime}}^{\infty}\right)^{\times} \rightarrow \mathcal{O}^{\times}$is a continuous character so that for each prime $v \mid l$, the action of the centre $\mathcal{O}_{F_{v}^{\prime}}^{\times}$of $\mathcal{O}_{B, v}^{\times}$on 
$W_{\lambda_{v}^{\prime}} \otimes_{\mathcal{O}} W_{\tau_{v}}$ is given by $\left.\left(\psi^{\prime}\right)^{-1}\right|_{\mathcal{O}_{F_{v}^{\prime}}^{\times}}$. The existence of such a character implies that there exists an integer $w^{\prime}$ such that $w^{\prime}=\lambda_{v, 1}^{\prime}+\lambda_{v, 2}^{\prime}+1$ for each $v \mid l$.

Let $U=\prod_{v} U_{v} \subset\left(B \otimes_{\mathbb{Q}} \mathbb{A}^{\infty}\right)^{\times}$be a compact open subgroup with $U_{v} \subset \mathcal{O}_{B, v}^{\times}$ for all $v$ and $U_{v}=\mathcal{O}_{B, v}^{\times}$for $v \mid l$. We let $S_{\lambda^{\prime}, \tau, \psi^{\prime}}(U, \mathcal{O})$ denote the space of functions

$$
f: B^{\times} \backslash\left(B \otimes \mathbb{Q} \mathbb{A}^{\infty}\right)^{\times} \rightarrow W_{\lambda^{\prime}, \tau}
$$

with $f(g u)=\left(\lambda^{\prime} \otimes \tau\right)\left(u_{l}\right)^{-1} f(g)$ and $f(g z)=\psi^{\prime}(z) f(g)$ for all $u \in U, z \in\left(\mathbb{A}_{F^{\prime}}^{\infty}\right)^{\times}$ and $g \in\left(B \otimes_{\mathbb{Q}} \mathbb{A}^{\infty}\right)^{\times}$. Writing $U=U^{l} \times U_{l}$, we let

$$
S_{\lambda^{\prime}, \tau, \psi^{\prime}}\left(U_{l}, \mathcal{O}\right)=\underset{U^{l}}{\lim _{\lambda^{\prime}}} S_{\lambda^{\prime}, \tau, \psi^{\prime}}\left(U^{l} \times U_{l}, \mathcal{O}\right)
$$

and we let $\left(B \otimes_{\mathbb{Q}} \mathbb{A}^{l, \infty}\right)^{\times}$act on this space by right translation.

Let $\psi_{\mathbb{C}}^{\prime}:\left(F^{\prime}\right)^{\times} \backslash \mathbb{A}_{F^{\prime}}^{\times} \rightarrow \mathbb{C}^{\times}$be the algebraic Hecke character defined by

$$
z \mapsto \mathbf{N}_{F^{\prime} / \mathbb{Q}}\left(z_{\infty}\right)^{1-w^{\prime}} \cdot \iota\left(\mathbf{N}_{F^{\prime} / \mathbb{Q}}\left(z_{l}\right)^{w^{\prime}-1} \psi^{\prime}\left(z^{\infty}\right)\right) .
$$

Let $W_{\tau, \mathbb{C}}=W_{\tau} \otimes_{\mathcal{O}, \iota} \mathbb{C}$. We have an isomorphism of $\left(B \otimes_{\mathbb{Q}} \mathbb{A}^{l, \infty}\right)^{\times}$-modules

$$
S_{\lambda^{\prime}, \tau, \psi^{\prime}}\left(U_{l}, \mathcal{O}\right) \otimes \mathcal{O}_{, \iota} \mathbb{C} \stackrel{\sim}{\longrightarrow} \bigoplus_{\Pi} \operatorname{Hom}_{\mathcal{O}_{B, l}^{\times}}\left(W_{\tau, \mathbb{C}}^{\vee}, \Pi_{l}\right) \otimes \Pi^{\infty, l},
$$

where the sum is over all automorphic representations $\Pi$ of $(B \otimes \mathbb{Q} \mathbb{A})^{\times}$of weight $\iota_{*} \lambda^{\prime}:=\left(\lambda_{\iota^{-1} \sigma}^{\prime}\right)_{\sigma} \in\left(\mathbb{Z}_{+}^{2}\right)^{\operatorname{Hom}\left(F^{\prime}, \mathbb{C}\right)}$ and central character $\psi_{\mathbb{C}}^{\prime}$ (see for instance the proof of Lemma 1.3 of Tay06).

Let $U$ be as above and let $R$ denote a finite set of places of $F^{\prime}$ containing all those places $v$ where $U_{v} \neq \mathcal{O}_{B, v}^{\times}$. Let $\mathbb{T}^{\Sigma \cup R}$ denote the polynomial algebra $\mathcal{O}\left[T_{v}, S_{v}\right]$, where $v$ runs over all places of $F^{\prime}$ away from $l, R$ and $\Sigma$. For such a $v$ we let $T_{v}$ and $S_{v}$ act on $S_{\lambda^{\prime}, \tau, \psi^{\prime}}(U, \mathcal{O})$ via the double coset operators

$$
\left[U i_{v}^{-1}\left(\begin{array}{cc}
\varpi_{v} & 0 \\
0 & 1
\end{array}\right) U\right] \text { and }\left[U i_{v}^{-1}\left(\begin{array}{cc}
\varpi_{v} & 0 \\
0 & \varpi_{v}
\end{array}\right) U\right]
$$

respectively, where $\varpi_{v}$ is a uniformizer in $\mathcal{O}_{F_{v}^{\prime}}$.

Let $\widetilde{\pi}$ denote the automorphic representation of $\left(B \otimes_{\mathbb{Q}} \mathbb{A}\right)^{\times}$of weight $\lambda_{F^{\prime}}:=$ $\left(\lambda_{\left.\sigma\right|_{F}}\right)_{\sigma} \in\left(\mathbb{Z}_{+}^{2}\right)^{\operatorname{Hom}\left(F^{\prime}, \mathbb{C}\right)}$ corresponding to $\pi_{F^{\prime}}$ under the Jacquet-Langlands correspondence. Let $\iota^{*} \lambda=\left(\left(\lambda_{F^{\prime}}\right)_{\iota v}\right)_{v} \in\left(\mathbb{Z}_{+}^{2}\right)^{\operatorname{Hom}\left(F^{\prime}, \overline{\mathbb{Q}}_{l}\right)}$. Let $U=\prod_{v} U_{v} \subset\left(B \otimes \mathbb{Q} \mathbb{A}^{\infty}\right)^{\times}$ be the compact open subgroup with $U_{v}=\mathcal{O}_{B, v}^{\times}$for all $v$. Then the space $\widetilde{\pi}^{U}$ is non-zero. Let $\chi: G_{F^{\prime}}^{\mathrm{ab}} \rightarrow \overline{\mathbb{Q}}_{l}^{\times}$denote the character $\left.\epsilon \operatorname{det} r_{l, \iota}(\pi)\right|_{G_{F^{\prime}}}$ and let $\psi=\chi \circ \operatorname{Art}_{F^{\prime}}: \mathbb{A}_{F^{\prime}}^{\times} \overline{\left(F_{\infty}^{\prime}\right)_{>0}^{\times}\left(F^{\prime}\right)^{\times}} \rightarrow \overline{\mathbb{Q}}_{l}$. Note that $\chi$ is totally even and hence we may regard $\psi$ as a character of $\left(\mathbb{A}_{F^{\prime}}^{\infty}\right)^{\times} /\left(F^{\prime}\right)^{\times} \stackrel{\sim}{\longrightarrow} \mathbb{A}_{F^{\prime}}^{\times} \overline{\left(F_{\infty}^{\prime}\right)^{\times}\left(F^{\prime}\right)^{\times}}$. Extending $K$ if necessary, we can and do assume that $\psi$ is valued in $\mathcal{O}^{\times}$. Further extending $K$ if

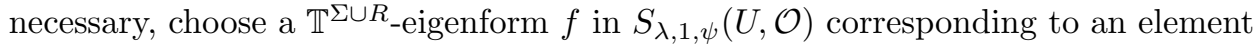
of $\left(\widetilde{\pi}^{\infty}\right)^{U}$ under the isomorphism (17.1.1). The $\mathbb{T}^{\Sigma \cup R}$-eigenvalues on $f$ give rise to an $\mathcal{O}$-algebra homomorphism $\mathbb{T}^{\Sigma \cup R} \rightarrow \mathcal{O}$ and reducing this modulo $\mathfrak{m}_{\mathcal{O}}$ gives rise to a maximal ideal $\mathfrak{m}$ of $\mathbb{T}^{\Sigma \cup R}$.

Let $\tilde{\chi}: G_{F^{\prime}}^{\mathrm{ab}} \rightarrow \mathcal{O}^{\times}$denote the Teichmüller lift of the reduction of $\chi$. Let $\psi^{\prime}=\tilde{\chi} \circ \operatorname{Art}_{F^{\prime}}$, which we can regard as a character $\left(\mathbb{A}_{F^{\prime}}^{\infty}\right)^{\times} /\left(F^{\prime}\right)^{\times} \rightarrow \mathcal{O}^{\times}$. Let $v$ be a place of $F^{\prime}$ dividing $l$. 
- If $\left.r_{l, \iota}(\pi)\right|_{G_{F_{v}^{\prime}}}$ is ordinary and $\iota^{*} \lambda_{v, 1} \neq \iota^{*} \lambda_{v, 2}$, let $\chi_{1}, \chi_{2}: \mathbb{F}_{l}^{\times} \rightarrow \mathbb{Q}_{l}^{\times}$be the characters given by $\chi_{1}(x)=\widetilde{x}^{\iota^{*} \lambda_{v, 1}}$ and $\chi_{2}(x)=\widetilde{x}^{\iota^{*} \lambda_{v, 2}}$, where $\widetilde{x}$ denotes the Teichmüller lift of $x$. Then let $\tau_{v}$ denote the representation

$$
I\left(\chi_{1}, \chi_{2}\right):=\operatorname{Ind}_{B\left(\mathbb{F}_{l}\right)}^{\mathrm{GL}_{2}\left(\mathbb{F}_{l}\right)}\left(\chi_{1} \otimes \chi_{2}\right)
$$

of $\mathrm{GL}_{2}\left(\mathbb{F}_{l}\right)$, where $B$ is the Borel subgroup of upper triangular matrices in $\mathrm{GL}_{2}$.

- If $\left.r_{l, \iota}(\pi)\right|_{G_{F_{v}^{\prime}}}$ is ordinary and $\iota^{*} \lambda_{v, 1}=\iota^{*} \lambda_{v, 2}$, let $\chi: \mathbb{F}_{l}^{\times} \rightarrow \mathbb{Q}_{l}^{\times}$be the character $\chi(x)=\widetilde{x}^{\iota^{*} \lambda_{v, 2}}$. Let $\tau_{v}$ denote the representation $\chi \circ \operatorname{det}$ of $\mathrm{GL}_{2}\left(\mathbb{F}_{l}\right)$.

- If $\left.r_{l, l}(\pi)\right|_{G_{F^{\prime}}}$ is not ordinary, let $\chi: \mathbb{F}_{l^{2}} \rightarrow \mathbb{Q}_{l^{2}}^{\times}$be the character given by $\chi(x)=\widetilde{x}^{\iota^{*} \lambda_{v, 1}-\iota^{*} \lambda_{v, 2}+2+(l+1)\left(\iota^{*} \lambda_{v, 2}-1\right)}$. Let $\tau_{v}$ be the $\overline{\mathbb{Q}}_{l}$-representation of $\mathrm{GL}_{2}\left(\mathbb{F}_{l}\right)$, denoted by $\Theta(\chi)$ in Section 3 of [CDT99] (note that $\chi^{l} \neq \chi$ since $\left.0<\iota^{*} \lambda_{v, 1}-\iota^{*} \lambda_{v, 2}+2<l+1\right)$.

Extending $K$ if necessary, we can and do fix a model for $\tau_{v}$ on a finite free $\mathcal{O}$-module $W_{\tau_{v}}$. We also view $W_{\tau_{v}}$ as a smooth $U_{v}=\mathcal{O}_{B, v^{-}}^{\times}$module via $U_{v} \stackrel{i_{v}}{\longrightarrow} \mathrm{GL}_{2}\left(\mathcal{O}_{F_{v}^{\prime}}\right) \stackrel{v}{\longrightarrow}$ $\mathrm{GL}_{2}\left(\mathbb{Z}_{l}\right) \rightarrow \mathrm{GL}_{2}\left(\mathbb{F}_{l}\right)$. By Lemma 3.1.1 of CDT99, $W_{\lambda_{v}} \otimes_{\mathcal{O}} k$ is a Jordan-Hölder constituent of $W_{\tau_{v}} \otimes_{\mathcal{O}} k$.

It follows that $W_{\lambda} \otimes_{\mathcal{O}} k$ is a Jordan-Hölder constituent of the $U_{l}$-module $W_{\tau} \otimes_{\mathcal{O}} k$. Also, since $l>3$ and $l$ is unramified in $F^{\prime}, B^{\times}$contains no elements of exact order $l$ and hence the group $U$ satisfies hypothesis 3.1.2 of [Kis09] (with $l$ replacing $p$ ). We can therefore apply Lemma 3.1.4 of [Kis09] to deduce that $\mathfrak{m}$ is in the support of $S_{0, \tau, \psi^{\prime}}(U, \mathcal{O})$. Let $\Pi^{\prime}$ denote the automorphic representation of $(B \otimes \mathbb{Q} \mathbb{A})^{\times}$

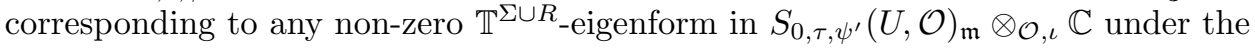
isomorphism (7.1.1). Let $\pi^{\prime}$ be the automorphic representation of $\mathrm{GL}_{2}\left(\mathbb{A}_{F^{\prime}}\right)$ corresponding to $\Pi^{\prime}$ under the Jacquet-Langlands correspondence. Then $\pi^{\prime}$ is regular algebraic and of weight 0 by construction. The choice of $\mathfrak{m}$ ensures that property (2) of the theorem holds and hence that $\pi^{\prime}$ is cuspidal. Property (3) holds by the choice of $U$.

It remains to show that $\pi^{\prime}$ satisfies property (4). Let $v$ be a place of $F^{\prime}$ dividing $l$ and suppose firstly that $\left.r_{l, l}(\pi)\right|_{G_{F_{v}^{\prime}}}$ is non-ordinary. Then, by the choice of $\tau_{v}$ and part (3) of Lemma 4.2.4 of [CDT99], we see that $\left.r_{l, \iota}\left(\pi^{\prime}\right)\right|_{G_{F_{v}^{\prime}}}$ is potentially Barsotti-Tate and moreover

$$
\left.\mathrm{WD}\left(\left.r_{l, L}\left(\pi^{\prime}\right)\right|_{G_{F_{v}^{\prime}}}\right)\right|_{I_{F_{v}^{\prime}}} \cong \widetilde{\omega}_{2}^{-\iota^{*} \lambda_{v, 1}-l \iota^{*} \lambda_{v, 2}+l-1} \oplus \widetilde{\omega}_{2}^{-l \iota^{*} \lambda_{v, 1}-\iota^{*} \lambda_{v, 2}+1-l},
$$

where $\omega_{2}$ is a fundamental character of niveau 2. We deduce that $\left.r_{l, \iota}\left(\pi^{\prime}\right)\right|_{G_{F_{v}^{\prime}}}$ only becomes Barsotti-Tate over a non-abelian extension of $G_{F_{v}^{\prime}}$ and hence is nonordinary. Now suppose that $\left.r_{l, \iota}(\pi)\right|_{G_{F^{\prime}}}$ is ordinary. Then by the choice of $\tau_{v}$ and parts (1) and (2) of Lemma 4.2.4 of [CDT99] we see that $\left.r_{l, \iota}\left(\pi^{\prime}\right)\right|_{G_{F_{v}^{\prime}}}$ is potentially Barsotti-Tate and moreover

$$
\left.\mathrm{WD}\left(\left.r_{l, \iota}\left(\pi^{\prime}\right)\right|_{G_{F_{v}^{\prime}}}\right)\right|_{I_{F_{v}^{\prime}}} \cong \widetilde{\omega}^{-\iota^{*} \lambda_{v, 1}} \oplus \widetilde{\omega}^{-l^{*} \lambda_{v, 2}},
$$

where $\omega$ is the mod $l$ cyclotomic character. Since $\left.\bar{r}_{l, L}(\pi)\right|_{G_{F_{v}^{\prime}}}$ is reducible, it follows from Theorem 6.11(3) of [Sav05] that $\left.r_{l, \iota}\left(\pi^{\prime}\right)\right|_{G_{F_{v}^{\prime}}}$ is either decomposable (in which case it is easy to see that it must be ordinary), or it corresponds to a potentially crystalline representation as in Proposition 2.17 of [Sav05, with $v_{l}\left(x_{1}\right)=1$ or $v_{l}\left(x_{2}\right)=1$ 
(because if neither of these hold, then by Theorem 6.11(3) of [Sav05] the representation $\left.\bar{r}_{l, \iota}\left(\pi^{\prime}\right)\right|_{G_{F_{v}^{\prime}}}=\left.\bar{r}_{l, \iota}(\pi)\right|_{G_{F_{v}^{\prime}}}$ would be irreducible, a contradiction). In either case the representation is ordinary (for example by Lemma 2.2 of [BLGHT09]).

We would like to thank Richard Taylor for pointing out the following lemma to us.

Lemma 7.1.2. Let $F$ be a totally real field, and let $\pi$ be a $R A E S D C$ representation of $\mathrm{GL}_{n}\left(\mathbb{A}_{F}\right)$. Let $l$ be a prime number, and fix an isomorphism $\iota: \overline{\mathbb{Q}}_{l} \rightarrow \mathbb{C}$. Suppose that for some place $v \nmid l$ of $F$, the Galois representation $\left.r_{l, L}(\pi)\right|_{G_{F v}}$ is pure. Then we have

$$
\mathrm{WD}\left(\left.r_{l, \iota}(\pi)\right|_{G_{F_{v}}}\right)^{F-s s}=\iota^{-1}\left(\operatorname{rec}\left(\pi_{v}\right) \otimes\left|\operatorname{Art}_{F_{v}}^{-1}\right|_{F_{v}}^{(1-n) / 2}\right),
$$

where $\mathrm{WD}\left(\left.r_{l, l}(\pi)\right|_{G_{F_{v}}}\right)$ denotes the Weil-Deligne representation associated to $\left.r_{l, \iota}(\pi)\right|_{G_{F_{v}}}$.

Proof. By Theorem 1.1 of [BLGHT09, the claimed equality holds on the Weil group (but we do not necessarily know that the monodromy is the same on each side). However, $\pi_{v}$ is generic (because $\pi$ is cuspidal) and we claim $\iota \mathrm{rec}^{-1}\left(\mathrm{WD}\left(\left.r_{l, \iota}(\pi)\right|_{G_{F_{v}}}\right)^{F-s s}\right)$ is also generic. To see this, first note that it is (essentially) tempered, by Lemma 1.4(3) of [TY07, and thus a subquotient of a unitary induction of a square-integrable representation of a Levi subgroup, by Theorem 2.3 of BW00].

Any such induction is irreducible (cf. page 72 of [DKV84]), and the fact that $\iota \mathrm{rec}^{-1}\left(\mathrm{WD}\left(\left.r_{l, \iota}(\pi)\right|_{G_{F_{v}}}\right)^{F-s s}\right)$ is generic then follows from Theorem 9.7 of Zel80.

The claimed equality follows (because a generic representation is determined by its supercuspidal support - this follows easily from the results of Zelevinsky recalled on page 36 of [HT01]).

Theorem 7.1.3. Continue using the setup at the beginning of this section, and let $\pi^{\prime}$ be as in Proposition 7.1.1. Let $N / F^{\prime}$ be a finite extension of number fields. There is a finite Galois extension of totally real fields $F^{\prime \prime} / F^{\prime}$ such that:

(1) $F^{\prime \prime}$ is linearly disjoint from $\bar{F}^{\mathrm{ker} \bar{r}_{l, L}\left(\pi^{\prime}\right)}\left(\zeta_{l}\right) N$ over $F^{\prime}$.

(2) There is a RAESDC automorphic representation $\pi_{n}^{\prime}$ of $\mathrm{GL}_{n}\left(\mathbb{A}_{F^{\prime \prime}}\right)$ of weight 0 and level prime to $l$ such that $\left.r_{l, L}\left(\pi_{n}^{\prime}\right) \cong \operatorname{Sym}^{n-1} r_{l, L}\left(\pi^{\prime}\right)\right|_{G_{F^{\prime \prime}}}$.

Proof. The central character $\omega_{\pi^{\prime}}$ of $\pi^{\prime}$ has finite order and is trivial at the infinite places, so we can choose a quadratic totally real extension $F_{1}$ of $F^{\prime}$ linearly disjoint from $N \bar{F}^{\operatorname{ker} \bar{r}_{l, L}\left(\pi^{\prime}\right)}\left(\zeta_{l}\right)$ (which we will henceforth call $F^{(\text {avoid) }}$ ) over $F^{\prime}$, such that if $\pi_{F_{1}}^{\prime}=B C_{F_{1} / F}\left(\pi^{\prime}\right)$, then $\omega_{\pi_{F_{1}}^{\prime}}$ has a square root (note that the obstruction to taking a square root is in the 2-torsion of the Brauer group of $F^{\prime}$ ). Say $\chi^{2}=\omega_{\pi_{F_{1}}^{\prime}}$, and write $\pi^{\prime \prime}=\pi_{F_{1}}^{\prime} \otimes\left(\chi^{-1} \circ\right.$ det$)$. Making a further solvable base change (and keeping $F_{1}$ linearly disjoint from $F^{\text {(avoid) }}$ over $F^{\prime}$ ), we may assume in addition that $\pi^{\prime \prime}$ has level prime to $l$ (that this is possible follows from local-global compatibility and Proposition 7.1.1(4)).

Then choose a rational prime $l^{\prime} \neq l$ and an isomorphism $\iota^{\prime}: \overline{\mathbb{Q}}_{l^{\prime}} \stackrel{\sim}{\longrightarrow} \mathbb{C}$ such that:

- $\pi_{v}^{\prime \prime}$ is unramified for all $v \mid l^{\prime}$.

- $l^{\prime}$ is unramified in $F_{1}$.

- $l^{\prime}>4(n-1)+1$.

- $l^{\prime}$ splits completely in the field of coefficients of $\pi^{\prime \prime}$. 
- The residual representation $\bar{r}_{l^{\prime}, \iota^{\prime}}\left(\pi^{\prime \prime}\right): G_{F_{1}} \rightarrow \mathrm{GL}_{2}\left(\overline{\mathbb{F}}_{l^{\prime}}\right)$ has a large image, in the sense that there are finite fields $\mathbb{F}_{l^{\prime}} \subset k \subset k^{\prime}$ with

$$
\mathrm{SL}_{2}(k) \subset \bar{r}_{l^{\prime}, \iota^{\prime}}\left(\pi^{\prime \prime}\right)\left(G_{F_{1}}\right) \subset k^{\prime \times} \mathrm{GL}_{2}(k) .
$$

(Note that this is automatically the case for all sufficiently large $l^{\prime}$ by Proposition 0.1 of [Dim05].) Coupled with the previous point, this in fact means that

$$
\mathrm{SL}_{2}\left(\mathbb{F}_{l^{\prime}}\right) \subset \bar{r}_{l^{\prime}, \iota^{\prime}}\left(\pi^{\prime \prime}\right)\left(G_{F_{1}}\right) \subset \mathrm{GL}_{2}\left(\mathbb{F}_{l^{\prime}}\right) .
$$

Since $\pi^{\prime \prime}$ has trivial central character, $\operatorname{det} r_{l^{\prime}, \iota^{\prime}}\left(\pi^{\prime \prime}\right)=\epsilon_{l}^{-1}$, and we can apply Proposition 6.1.3 to find a Galois extension $F_{2} / F_{1}$, linearly disjoint from $F^{\text {(avoid) }}$ over $F^{\prime}$, such that $\mathrm{Sym}^{n-1} r_{l^{\prime}, \iota^{\prime}}\left(\pi^{\prime \prime}\right)$ is automorphic over $F_{2}$ of weight 0 . That it is in fact automorphic of level prime to $l$ follows from Lemma 7.1.2 (note that $\left.\operatorname{Sym}^{n-1} r_{l^{\prime}, \iota^{\prime}}\left(\pi^{\prime \prime}\right)\right|_{G_{F_{2}}}$ is pure, because $r_{l^{\prime}, \iota^{\prime}}\left(\pi^{\prime \prime}\right)$ is pure, for example by the main result of [Bla06], and $\left.\mathrm{Sym}^{n-1} r_{l^{\prime}, \iota^{\prime}}\left(\pi^{\prime \prime}\right)\right|_{G_{F_{2}}}$ is unramified at all places dividing $l$ by the choice of $F_{2}$ ). Replacing $F_{2}$ by a further solvable base change, also disjoint from $F^{\text {(avoid) }}$ over $F^{\prime}$, if necessary, we may assume that $\chi$ is unramified at all places of $F_{2}$ lying over $l$. We are then done, taking $F^{\prime \prime}=F_{2}$ (because $\left.\operatorname{Sym}^{n-1} r_{l^{\prime}, \iota^{\prime}}\left(\pi^{\prime}\right)\right|_{G_{F_{2}}}=$ $\left.\left.\left.r_{l^{\prime}, \iota^{\prime}}(\chi)^{n-1}\right|_{G_{F_{2}}} \otimes \operatorname{Sym}^{n-1} r_{l^{\prime}, \iota^{\prime}}\left(\pi^{\prime \prime}\right)\right|_{G_{F_{2}}}\right)$.

Theorem 7.1.4. Let $F$ be a totally real field, and let $\pi$ be a non-CM regular algebraic cuspidal automorphic representation of $\mathrm{GL}_{2}\left(\mathbb{A}_{F}\right)$. Then there is a prime l, an isomorphism $\overline{\mathbb{Q}}_{l} \stackrel{\sim}{\longrightarrow} \mathbb{C}$, a finite Galois extension of totally real fields $F^{\prime \prime} / F$ and an $R A E S D C$ automorphic representation $\pi_{n}$ of $\mathrm{GL}_{n}\left(\mathbb{A}_{F^{\prime \prime}}\right)$ such that $r_{l, \iota}\left(\pi_{n}\right) \cong$ $\left.\operatorname{Sym}^{n-1} r_{l, \iota}(\pi)\right|_{G_{F^{\prime \prime}}}$.

Proof. We continue to use the notation established above, and in particular we will fix $l$ and $\iota$ as above, and make use of $F^{\prime}$ and $\pi^{\prime} . F^{\prime \prime}$ will be as in the conclusion of Theorem 7.1.3, which we will apply with a particular choice of field $N$, to be determined below. We can and do assume that $r_{l, \iota}(\pi)$ and $r_{l, \iota}\left(\pi^{\prime}\right)$ both take values in $\mathrm{GL}_{n}(\mathcal{O})$, where $\mathcal{O}$ is the ring of integers in a finite extension $K$ of $\mathbb{Q}_{l}$ inside $\overline{\mathbb{Q}}_{l}$. Let $k$ denote the residue field of $K$. We can and do further assume that $\left.r_{l, \iota}(\pi)\right|_{G_{F^{\prime}}}$ and $r_{l, \iota}\left(\pi^{\prime}\right)$ are equal (as homomorphisms) when composed with the natural map $\mathrm{GL}_{n}(\mathcal{O}) \rightarrow \mathrm{GL}_{n}(k)$. Write $\left.\bar{r}_{l, \iota}(\pi)\right|_{G_{F^{\prime}}}=\bar{r}_{l, \iota}\left(\pi^{\prime}\right)$ for this composition.

We write $r=\left.\operatorname{Sym}^{n-1} r_{l, \iota}(\pi)\right|_{G_{F^{\prime}}}$ and $r^{\prime}=\operatorname{Sym}^{n-1} r_{l, \iota}\left(\pi^{\prime}\right)$, thought of as representations valued in $\mathrm{GL}_{n}(\mathcal{O})$ via the bases specified in Definition 3.3.1. We begin by applying Lemma 4.2.4 in the following situation (where the field $F$ of Lemma 4.2 .4 is $\left.F^{\prime}\right)$ :

- $S_{\text {ord }}$ is the set of places of $F^{\prime}$ dividing $l$ lying over a place for which $\pi$ is ordinary with respect to $\iota$.

- For each $v \mid l$, thought of as an embedding $F^{\prime} \hookrightarrow \overline{\mathbb{Q}}_{l}, a_{v}=-\lambda_{\left.\iota \circ v\right|_{F}, 2}$ and $b_{v}=\lambda_{\left.\iota \circ v\right|_{F}, 1}-\lambda_{\left.\iota \circ v\right|_{F}, 2}+1$.

- $T$ is the set of places away from $l$ at which $\pi^{\prime}$ is ramified.

- $\left(F^{\prime}\right)^{(\mathrm{bad})}=\bar{F}^{\mathrm{ker} \bar{r}}\left(\zeta_{l}\right)$.

We deduce that (after possibly extending $K$ ) there is a CM extension $M$ of $F^{\prime}$ of degree $m^{*}$, and de Rham characters

$$
\theta, \theta^{\prime}: G_{M} \rightarrow \mathcal{O}^{\times},
$$


satisfying various properties that we will now describe. We can fix an element $\tilde{\tau} \in G_{F^{\prime}}$ mapping to a generator $\tau \in \operatorname{Gal}\left(M / F^{\prime}\right)$, and we regard $\operatorname{Ind}_{G_{M}}^{G_{F^{\prime}}} \theta$ and $\operatorname{Ind}_{G_{M}}^{G_{F^{\prime}}} \theta^{\prime}$ as representations valued in $\mathrm{GL}_{m^{*}}(\mathcal{O})$ via their $\tilde{\tau}$-standard bases $\beta=$ $\left\{e_{0}, \ldots, e_{m^{*}-1}\right\}$ and $\beta^{\prime}=\left\{e_{0}^{\prime}, \ldots, e_{m^{*}-1}^{\prime}\right\}$ respectively, in the sense of Definition 4.2.3. Note that these two representations become equal when composed with the homomorphism $\mathrm{GL}_{m^{*}}(\mathcal{O}) \rightarrow \mathrm{GL}_{m^{*}}(k)$. Then, by the conclusions of Lemma 4.2.4, the following hold:

- $\bar{\theta}=\bar{\theta}^{\prime}$.

- The image of $\left.\left(r \otimes \operatorname{Ind}_{G_{M}}^{G_{F^{\prime}}} \theta\right)\right|_{G_{F^{\prime}\left(\zeta_{l}\right)}}$ is big.

- $\bar{F}^{\left(\operatorname{ker} \operatorname{ad}\left(\bar{r} \otimes \operatorname{Ind}_{G_{M}}^{G_{F^{\prime}}} \bar{\theta}\right)\right)}$ does not contain $\zeta_{l}$.

- $r \otimes \operatorname{Ind}_{G_{M}}^{G_{F^{\prime}}} \theta$ and $r^{\prime} \otimes \operatorname{Ind}_{G_{M}}^{G_{F^{\prime}}} \theta^{\prime}$ are both de Rham and have the same HodgeTate weights at each place of $F^{\prime}$ dividing $l$.

- $r^{\prime \prime}:=r \otimes \operatorname{Ind}_{G_{M}}^{G_{F^{\prime}}} \theta$ is essentially self-dual; that is, there is a character $\chi: G_{F^{\prime}} \rightarrow \overline{\mathbb{Q}}_{l}^{\times}$with $\chi\left(c_{v}\right)$ independent of $v \mid \infty$ (where $c_{v}$ denotes a complex conjugation at $v$ ) such that $\left(r^{\prime \prime}\right)^{\vee} \cong r^{\prime \prime} \chi$.

- $\operatorname{Ind}_{G_{M}}^{G_{F^{\prime}}} \theta^{\prime}$ is essentially self-dual.

Applying Theorem 7.1 .3 with $N=\bar{M}^{\text {ker } \bar{\theta}}$, we find a totally real field $F^{\prime \prime} / F^{\prime}$ with $\left.r^{\prime}\right|_{G_{F^{\prime \prime}}}$ automorphic of level prime to $l$. By Proposition 5.1.3, the representation $\left.\left(r^{\prime} \otimes \operatorname{Ind}_{G_{M}}^{G_{F^{\prime}}} \theta^{\prime}\right)\right|_{G_{F^{\prime \prime}}}$ is automorphic. We now choose a solvable extension $F^{+} / F^{\prime \prime}$ of totally real fields such that:

- $F^{+}$is linearly disjoint from $\bar{F}^{\text {ker ad } \bar{r}^{\prime \prime}}\left(\zeta_{l}\right) M$ over $F^{\prime}$.

- $\left.r^{\prime \prime}\right|_{G_{F^{+}}}=\left.\left(r \otimes \operatorname{Ind}_{G_{M}}^{G_{F^{\prime}}} \theta\right)\right|_{G_{F^{+}}}$and $\left.\left(r^{\prime} \otimes \operatorname{Ind}_{G_{M}}^{G_{F^{\prime}}} \theta^{\prime}\right)\right|_{G_{F^{+}}}$are both crystalline at all places dividing $l$.

- $\left.\left(r^{\prime} \otimes \operatorname{Ind}_{G_{M}}^{G_{F^{\prime}}} \theta^{\prime}\right)\right|_{G_{F^{+}}}$is automorphic of level prime to $l$ (note that $\left.r^{\prime}\right|_{G_{F^{\prime \prime}}}$ is automorphic of level prime to $l$, so this is easily achieved by Lemma 5.1.1 and Proposition 5.1.3).

- The extension $F^{+} M / F^{+}$is unramified at all finite places and is split completely at all places of $F^{+}$lying over places in $T$.

- $\left.\theta\right|_{G_{F+} M}$ and $\left.\theta^{\prime}\right|_{G_{F^{+}} M}$ are both unramified at all places not dividing $l$.

- If $v \mid l$ is a place of $F^{+}$, then $F_{v}^{+}$contains the unramified quadratic extension of $F_{v}^{\prime}$, and $\left.\bar{r}^{\prime \prime}\right|_{G_{F_{v}^{+}}}$is trivial.

Write $\rho:=\left.r^{\prime \prime}\right|_{G_{F^{+}}}=\left.\left(r \otimes \operatorname{Ind}_{G_{M}}^{G_{F^{\prime}}} \theta\right)\right|_{G_{F^{+}}}, \rho^{\prime}:=\left.\left(r^{\prime} \otimes \operatorname{Ind}_{G_{M}}^{G_{F^{\prime}}} \theta^{\prime}\right)\right|_{G_{F^{+}}}: G_{F^{+}} \rightarrow$ $\mathrm{GL}_{n m^{*}}(\mathcal{O})$, so that $\rho^{\prime}$ is automorphic of level prime to $l$.

Sublemma. For each place $w \mid l$ of $F^{+},\left.\left.\rho\right|_{G_{F_{w}^{+}}} \sim \rho^{\prime}\right|_{G_{F_{w}^{+}}}$.

Proof. If $w$ lies over a place of $S_{\text {ord }}$, this follows from Lemma 3.4.5. Otherwise, $w$ lies over a place $v$ in $S_{\mathrm{ss}}$. Let $L$ be the unramified quadratic extension of $F_{v}^{\prime}$ in $\bar{F}_{v}^{\prime}$. Then $\left.\bar{r}_{l, L}(\pi)\right|_{G_{F_{v}^{\prime}}} \cong \operatorname{Ind}_{G_{L}}^{G_{F_{v}^{\prime}}} \bar{\chi}$ for some character $\bar{\chi}: G_{L} \rightarrow k^{\times}$(see for example Théorème 3.2.1(1) of [Ber10]). We can and do (after possibly extending $K$ ) choose a crystalline lift $\chi: G_{L} \rightarrow \mathcal{O}^{\times}$of $\bar{\chi}$ with Hodge-Tate weights $-a_{v}$ and $b_{v}-a_{v}$ (recall that $l$ splits completely in $F^{\prime}$ ). We can also (again, extending $K$ if necessary) choose a de Rham character $\chi^{\prime}: G_{L} \rightarrow \mathcal{O}^{\times}$lifting $\bar{\chi}$ with Hodge-Tate weights 0 and 1 , which becomes crystalline over $F_{w}^{+}$(we can do this by the assumption that $\left.\bar{r}^{\prime \prime}\right|_{G_{F_{w}^{+}}}$ is trivial). 
Choose an element $\sigma \in G_{F_{v}^{\prime}}$ mapping to a generator of $\operatorname{Gal}\left(L / F_{v}^{\prime}\right)$ and fix the $\sigma$-standard (in the sense of Definition 4.2.3) bases of $\operatorname{Ind}_{G_{L}}^{G_{F_{v}^{\prime}}} \chi, \operatorname{Ind}_{G_{L}}^{G_{F_{v}^{\prime}}} \chi^{\prime}$, $\operatorname{Sym}^{n-1} \operatorname{Ind}_{G_{L}}^{G_{F_{v}^{\prime}}} \chi$, and $\operatorname{Sym}^{n-1} \operatorname{Ind}_{G_{L}}^{G_{F_{v}^{\prime}}} \chi^{\prime}$.

Choose a matrix $A \in \mathrm{GL}_{n}(\mathcal{O})$ with $A\left(\operatorname{Ind}_{G_{L}}^{G_{F_{v}^{\prime}}} \bar{\chi}\right) A^{-1}=\left.\bar{r}_{l, \iota}(\pi)\right|_{G_{F_{v}^{\prime}}}$ and write $r_{\chi}=$ $A\left(\operatorname{Ind}_{G_{L}}^{G_{F_{v}^{\prime}}} \chi\right) A^{-1}$. We have $\left.r_{\chi} \sim r_{l, \iota}(\pi)\right|_{G_{F_{v}^{\prime}}}$, because both liftings are crystalline of the same weight, $F_{v}$ is unramified, and the common weight is in the FontaineLaffaille range (see e.g. Lemma 2.4.1 of [CHT08, which shows that the appropriate lifting ring is formally smooth over $\mathcal{O}$ ). Then by the remarks following Definition 3.3.5, we have $\left.\left.\left(r_{\chi}\right)\right|_{G_{F_{w}^{+}}} \sim r_{l, \iota}(\pi)\right|_{G_{F_{w}^{+}}}$, and $\left.\left.\operatorname{Sym}^{n-1}\left(r_{\chi}\right)\right|_{G_{F_{w}^{+}}} \sim \operatorname{Sym}^{n-1} r_{l, \iota}(\pi)\right|_{G_{F_{w}^{+}}}$, so that (with the inherited bases)

$$
\left.\left.\left(\left.\operatorname{Sym}^{n-1}\left(r_{\chi}\right)\right|_{G_{F_{w}^{+}}}\right) \otimes\left(\operatorname{Ind}_{G_{M}}^{G_{F^{\prime}}} \theta\right)\right|_{G_{F_{w}^{+}}} \sim\left(\left.\operatorname{Sym}^{n-1} r_{l, \iota}(\pi)\right|_{G_{F_{w}^{+}}}\right) \otimes\left(\operatorname{Ind}_{G_{M}}^{G_{F^{\prime}}} \theta\right)\right|_{G_{F_{w}^{+}}} .
$$

Similarly, write $r_{\chi^{\prime}}=A\left(\operatorname{Ind}_{G_{L}}^{G_{F_{v}^{\prime}}} \chi^{\prime}\right) A^{-1}$. Then $\left.\left.r_{\chi^{\prime}}\right|_{G_{F_{w}^{+}}} \sim r_{l, \iota}\left(\pi^{\prime}\right)\right|_{G_{F_{w}^{+}}}$, because both representations are Barsotti-Tate and non-ordinary (see Proposition 2.3 of Gee06, which shows that all non-ordinary points lie on the same component of the appropriate lifting ring). Then $\left.\left.\operatorname{Sym}^{n-1}\left(r_{\chi^{\prime}}\right)\right|_{G_{F_{w}^{+}}} \sim \operatorname{Sym}^{n-1} r_{l, \iota}\left(\pi^{\prime}\right)\right|_{G_{F_{w}^{+}}}$and

$$
\left.\left.\left(\left.\operatorname{Sym}^{n-1}\left(r_{\chi^{\prime}}\right)\right|_{G_{F_{w}^{+}}}\right) \otimes\left(\operatorname{Ind}_{G_{M}}^{G_{F^{\prime}}} \theta^{\prime}\right)\right|_{G_{F_{w}^{+}}} \sim\left(\left.\operatorname{Sym}^{n-1} r_{l, \iota}\left(\pi^{\prime}\right)\right|_{G_{F_{w}^{+}}}\right) \otimes\left(\operatorname{Ind}_{G_{M}}^{G_{F^{\prime}}} \theta^{\prime}\right)\right|_{G_{F_{w}^{+}}} .
$$

By Lemma 4.2.5, we have

$$
\left.\left.\left(\left.\operatorname{Sym}^{n-1}\left(r_{\chi^{\prime}}\right)\right|_{G_{F_{w}^{+}}}\right) \otimes\left(\operatorname{Ind}_{G_{M}}^{G_{F^{\prime}}} \theta^{\prime}\right)\right|_{G_{F_{w}^{+}}} \sim\left(\left.\operatorname{Sym}^{n-1}\left(r_{\chi}\right)\right|_{G_{F_{w}^{+}}}\right) \otimes\left(\operatorname{Ind}_{G_{M}}^{G_{F^{\prime}}} \theta\right)\right|_{G_{F_{w}^{+}}} .
$$

Since

$$
\begin{aligned}
\left.\rho\right|_{G_{F_{w}^{+}}} & =\left.\left(\left.\operatorname{Sym}^{n-1} r_{l, \iota}(\pi)\right|_{G_{F_{w}^{+}}}\right) \otimes\left(\operatorname{Ind}_{G_{M}}^{G_{F^{\prime}}} \theta\right)\right|_{G_{F_{w}^{+}}}, \\
\left.\rho^{\prime}\right|_{G_{F_{w}^{+}}} & =\left.\left(\left.\operatorname{Sym}^{n-1} r_{l, \iota}\left(\pi^{\prime}\right)\right|_{G_{F_{w}^{+}}}\right) \otimes\left(\operatorname{Ind}_{G_{M}}^{G_{F^{\prime}}} \theta^{\prime}\right)\right|_{G_{F_{w}^{+}}},
\end{aligned}
$$

the result follows from the transitivity of $\sim$.

For $v$ a place of $F^{+}$, let $R_{\left.\bar{\rho}\right|_{G_{F_{v}}} ^{\square}}$ denote the universal $\mathcal{O}$-lifting ring of $\left.\bar{\rho}\right|_{G_{F_{v}^{+}}}$. Extending $\mathcal{O}$ if necessary, we can and do assume that if $v$ is such that at least one $\rho$ or $\rho^{\prime}$ is ramified at $v$, then for each minimal prime ideal $\wp$ of $R_{\bar{\rho}_{G_{F_{v}^{+}}}^{\square}}$, the quotient $R_{\left.\bar{\rho}\right|_{G_{F_{v}}} ^{\square}}^{\square} / \wp$ is geometrically integral.

Sublemma. For all places $v \nmid l$ of $F^{+}$, either

- $\left.\rho\right|_{G_{F_{v}^{+}}}$and $\left.\rho^{\prime}\right|_{G_{F_{v}^{+}}}$are both unramified, or

- each of the following conditions holds:

- $\left.\left.\rho^{\prime}\right|_{G_{F_{v}^{+}}} \rightsquigarrow \mathcal{O} \rho\right|_{G_{F_{v}^{+}}}$(see Definition 3.3.7),

$-\left.\left.\rho\right|_{G_{F_{v}^{+}}} \rightsquigarrow \mathcal{O} \rho^{\prime}\right|_{G_{F_{v}^{+}}}$, and

- the similitude characters of $\rho$ and $\rho^{\prime}$ agree on inertia at $v$.

Proof. If $v$ does not lie over a place in $T$, then there is nothing to prove, so we may suppose that $v$ lies over a place of $T$. The condition on similitude characters is immediate since $\rho$ and $\rho^{\prime}$ are both unipotent on inertia at $v$. Let us then turn to checking the condition that $\left.\left.\rho^{\prime}\right|_{G_{F_{v}^{+}}} \rightsquigarrow \mathcal{O} \rho\right|_{G_{F_{v}^{+}}}$. By assumption, condition (1) 
in Definition 3.3.7 is satisfied, so we just need to check condition (2). Let $\rho_{v}=$ $\left.r_{l, \iota}(\pi)\right|_{G_{F_{v}^{+}}}$and $\rho_{v}^{\prime}=\left.r_{l, \iota}\left(\pi^{\prime}\right)\right|_{G_{F_{v}^{+}}}$. Then $\rho_{v}$ and $\rho_{v}^{\prime}$ are both lifts of the same reduction $\bar{\rho}_{v}: G_{F_{v}^{+}} \rightarrow \mathrm{GL}_{2}(k)$. It follows easily from Corollary 2.6.7 of Kis09] that there is a quotient $R_{\bar{\rho}_{v}}^{\mathrm{St}}$ of $R_{\bar{\rho}_{v}}^{\square}$ corresponding to lifts which are extensions of an unramified character $\gamma$ by $\gamma \epsilon$, and furthermore that the ring $R_{\bar{\rho}_{v}}^{\mathrm{St}}$ is an integral domain of dimension 5. Let $\rho_{v}^{\square}$ denote the universal lift to $R \bar{\rho}_{v}^{\mathrm{St}}$. Then $\rho_{v}$ and $\rho_{v}^{\prime}$ arise as specialisations of this lift at closed points of $R_{\bar{\rho}_{v}}^{\mathrm{St}}[1 / l]$; let us call these points $x$ and $x^{\prime}$.

Note that $\left.\left(\operatorname{Ind}_{G_{M}}^{G_{F^{\prime}}} \theta\right)\right|_{G_{F_{v}^{+}}}=\bigoplus_{i=0}^{m^{*}-1}\left(\left.\theta^{\tau^{i}}\right|_{G_{F_{v}}}\right) e_{i}$ and similarly $\left.\left(\operatorname{Ind}_{G_{M}}^{G_{F^{\prime}}} \theta^{\prime}\right)\right|_{G_{F_{v}^{+}}}=$ $\bigoplus_{i=0}^{m^{*}-1}\left(\left.\theta^{\prime \tau^{i}}\right|_{G_{F_{v}}}\right) e_{i}^{\prime}$. For $i=0, \ldots, m^{*}-1$, let $\tilde{\theta}_{i}: G_{F_{v}^{+}} \rightarrow \mathcal{O}^{\times}$denote the Teichmüller lift of $\left.\bar{\theta}^{\tau^{i}}\right|_{G_{F_{v}^{+}}}$. If $R$ is an object of $\mathcal{C}_{\mathcal{O}}$ and $r \in R^{\times}$, we let $\lambda(r)$ : $G_{F_{v}^{+}} \rightarrow R^{\times}$denote the unramified character sending Frob $F_{v}^{+}$to $r$.

Let $R=R_{\left.\bar{\rho}\right|_{G_{F_{v}}} ^{\square}}$ and let $S=R_{\bar{\rho}_{v}}^{\mathrm{St}}\left[\left[X_{0}, \ldots, X_{m^{*}-1}\right]\right]$. The lift

$$
\operatorname{Sym}^{n-1} \rho_{v}^{\square} \otimes\left(\bigoplus_{i=0}^{m^{*}-1} \widetilde{\theta}_{i} \lambda\left(1+X_{i}\right)\right) e_{i}
$$

of $\left.\bar{\rho}\right|_{G_{F_{v}^{+}}}$gives rise to a map $\operatorname{Spec} S \rightarrow \operatorname{Spec} R$. Since $S$ is a domain, the image of this map must be contained in an irreducible component of $\operatorname{Spec} R$. We deduce that $\left.\rho\right|_{G_{F^{+}}}$and $\left.\rho^{\prime}\right|_{G_{F^{+}}}$are contained in a common irreducible component of Spec $R[1 / l]$.

To prove that $x^{\prime}$ is contained in a unique irreducible component, it then suffices to prove that $x^{\prime}$ is a smooth point of Spec $R[1 / l]$. Since the completed local ring $R_{x^{\prime}}^{\wedge}$ at $x^{\prime}$ is the universal $K$-lifting ring of $\left(\left.\rho^{\prime}\right|_{G_{F_{v}^{+}}}\right) \otimes_{\mathcal{O}} K$, a standard argument shows that $R_{x^{\prime}}^{\wedge}$ is smooth if $H^{2}\left(G_{F_{v}^{+}}\right.$, ad $\left.\left.\rho^{\prime}\right|_{G_{F_{v}^{+}}}\right)=0$. By Tate local duality, it suffices to show that $H^{0}\left(G_{F_{v}^{+}}\right.$ad $\left.\left.\rho^{\prime}\right|_{G_{F_{v}^{+}}}(1)\right)=0$, i.e. that $\operatorname{Hom}_{G_{F_{v}^{+}}}\left(\left.\rho^{\prime}\right|_{G_{F_{v}^{+}}},\left.\rho^{\prime}\right|_{G_{F_{v}^{+}}}(1)\right)=0$.

Let $\mathrm{St}_{v}$ denote the $n$-dimensional representation of $G_{F_{v}^{+}}$corresponding to the Steinberg representation. Then $\left.\rho^{\prime}\right|_{G_{F_{v}^{+}}}$is $\mathrm{GL}_{n}(K)$-conjugate to an unramified twist of

$$
\left.\bigoplus_{i=0}^{m^{*}-1} \mathrm{St}_{v} \otimes \theta^{\prime \tau^{i}}\right|_{G_{F_{v}^{+}}}
$$

We may therefore assume that $\left.\rho^{\prime}\right|_{G_{F_{v}^{+}}}$is equal to this representation. It is easy to check (for example by considering the corresponding Weil-Deligne representation) that the representation $\mathrm{St}_{v}$ contains a unique $j$-dimensional subrepresentation for each $j$. Then a non-zero element of $\operatorname{Hom}_{G_{F_{v}^{+}}}\left(\left.\rho^{\prime}\right|_{G_{F_{v}^{+}}},\left.\rho^{\prime}\right|_{G_{F_{v}^{+}}}(1)\right)$ would have to give a non-zero map from the unique $j$-dimensional quotient of $\left.\mathrm{St}_{v} \otimes \theta^{\prime \tau^{i}}\right|_{G_{F_{v}^{+}}}$to the unique $j$-dimensional subrepresentation of $\left.\mathrm{St}_{v} \otimes \theta^{\prime \tau^{i^{\prime}}}(1)\right|_{G_{F_{v}^{+}}}$for some $i, i^{\prime}$ and $j$. This implies that $\left.\left(\theta^{\prime \tau^{i^{\prime}}} / \theta^{\prime \tau^{i}}\right)\right|_{G_{F_{v}^{+}}}$is a non-zero power of the cyclotomic character, which is impossible, because $\theta^{\prime \tau^{i^{\prime}}} / \theta^{\prime \tau^{i}}$ is a ratio of algebraic characters of the same weight, and is thus pure of weight 0 .

Finally, we can see that $\left.\left.\rho\right|_{G_{F_{v}^{+}}} \rightsquigarrow \mathcal{O} \rho^{\prime}\right|_{G_{F_{v}^{+}}}$using the same argument. 
By Theorem 3.6.2, $\rho$ is automorphic (the conditions on the image of $\bar{\rho}$ follow from Lemma 4.2.4 and the choice of $F^{+}$, and the remaining conditions follow by construction and the two sublemmas just proved). Since $F^{+} / F^{\prime \prime}$ is solvable and $\rho=\left.r^{\prime \prime}\right|_{G_{F^{+}}},\left.r^{\prime \prime}\right|_{G_{F^{\prime \prime}}}$ is automorphic by Lemma 1.3 of [BLGHT09]. But $\left.r^{\prime \prime}\right|_{G_{F^{\prime \prime}}}=$ $\left.\left.\left(\operatorname{Sym}^{n-1} r_{l, \iota}(\pi)\right)\right|_{G_{F^{\prime \prime}}} \otimes\left(\operatorname{Ind}_{G_{M}}^{G_{F}} \theta\right)\right|_{G_{F^{\prime \prime}}}=\left.\left(\operatorname{Sym}^{n-1} r_{l, \iota}(\pi)\right)\right|_{G_{F^{\prime \prime}}} \otimes\left(\operatorname{Ind}_{G_{M F^{\prime \prime}}}^{G_{F^{\prime \prime}}}\left(\left.\theta\right|_{G_{M F^{\prime \prime}}}\right)\right)$, so by Proposition 5.2.1. ( $\left.\operatorname{Sym}^{n-1} r_{l, \iota}(\pi)\right)\left.\right|_{G_{F^{\prime \prime}}}$ is automorphic, as required.

Corollary 7.1.5. Suppose that $F$ is a totally real field and that $\pi$ is a non-CM regular algebraic cuspidal automorphic representation of $\mathrm{GL}_{2}\left(\mathbb{A}_{F}\right)$ of weight $\lambda$, and let $w_{\pi}$ be the common value of the numbers $\lambda_{v, 1}+\lambda_{v, 2}, v \mid \infty$. Suppose that $n$ is a positive integer and that $\psi: F^{\times} \backslash \mathbb{A}_{F}^{\times} \rightarrow \mathbb{C}^{\times}$is a finite order character. Then there is a meromorphic function $L\left(\mathrm{Sym}^{n-1} \pi \times \psi, s\right)$ on the whole complex plane such that:

- For any prime $l$ and any isomorphism $\iota: \overline{\mathbb{Q}}_{l} \stackrel{\sim}{\longrightarrow} \mathbb{C}$ we have $L\left(\operatorname{Sym}^{n-1} \pi \times\right.$ $\psi, s)=L\left(\iota\left(\operatorname{Sym}^{n-1} r_{l, \iota}(\pi) \otimes r_{l, \iota}(\psi)\right), s\right)$.

- The expected functional equation holds between $L\left(\operatorname{Sym}^{n-1} \pi \times \psi, s\right)$ and $L\left(\operatorname{Sym}^{n-1}\left(\pi^{\vee}|\operatorname{det}|^{-w_{\pi}}\right) \times \psi, 1+(n-1) w_{\pi}-s\right)$.

- If $n>1$ or $\psi \neq 1$, then $L\left(\operatorname{Sym}^{n-1} \pi \times \psi, s\right)$ is holomorphic and non-zero in $\Re s \geq 1+(n-1) w_{\pi} / 2$.

Proof. This follows from Theorem 7.1.4, as in the proof of Theorem 4.2 of [HSBT10]. We give the details. The $L$-function $L\left(\iota\left(\operatorname{Sym}^{n-1} r_{l, \iota}(\pi) \otimes r_{l, \iota}(\psi)\right), s\right)$ is independent of the choice of $l, \iota$ by definition, so it is enough to prove the result for the $l, \iota$ fixed throughout this section. Let $\pi_{n}, F^{\prime \prime}$ be as in the conclusion of Theorem 7.1.4.

We claim that $\operatorname{rec}\left(\pi_{n, v}\right)=\left.\left(\operatorname{Sym}^{n-1} \operatorname{rec}\left(\pi_{v}\right)\right)\right|_{W_{F_{v}^{\prime \prime}}}$ for all places $v$ of $F^{\prime \prime}$. If $v \nmid l$, this follows from Lemma 7.1 .2 and the purity of $r_{l, \iota}(\pi)$ (which follows from the main result of [Bla06]). If $v \mid l$, then we choose a prime $p \neq l$ and an isomorphism $\iota^{\prime}: \overline{\mathbb{Q}}_{p} \stackrel{\sim}{\longrightarrow} \mathbb{C}$. By the Tchebotarev density theorem we have $r_{p, \iota^{\prime}}\left(\pi_{n}\right)=\left.\left(\operatorname{Sym}^{n-1} r_{p, \iota^{\prime}}(\pi)\right)\right|_{G_{F^{\prime \prime}}}$, and we may argue as before.

By Lemma 1.3 of [BLGHT09], for any intermediate field $F \subset F_{j} \subset F^{\prime \prime}$ with $F^{\prime \prime} / F_{j}$ soluble, there is an automorphic representation $\pi^{j}$ of $\mathrm{GL}_{n}\left(\mathbb{A}_{F_{j}}\right)$ with $r_{l, \iota}\left(\pi^{j}\right)$ $=\left.\left(\operatorname{Sym}^{n-1} r_{l, \iota}(\pi)\right)\right|_{G_{F_{j}}}$. By Brauer's theorem, we can write

$$
1=\sum_{j} a_{j} \operatorname{Ind}_{G_{F}}^{G_{F}} \chi_{j}
$$

with $a_{j} \in \mathbb{Z}$ and $\chi_{j}: G_{F_{j}} \rightarrow \mathbb{C}^{\times}$a homomorphism. Then by the above discussion (applied to the representations $\pi^{j}$ ), we have

$$
L\left(\iota\left(\operatorname{Sym}^{n-1} r_{l, \iota}(\pi) \otimes r_{l, \iota}(\psi)\right), s\right)=\prod_{j} L\left(\pi^{j} \otimes\left(\chi_{j} \circ \operatorname{Art}_{F_{j}}\right) \otimes \psi, s\right)^{a_{j}} .
$$

The result follows.

We now deduce the Sato-Tate conjecture for $\pi$, following the formulation of Gee09] (see also section 8 of [BLGHT09]). Recall that $w_{\pi}$ is the common value of the $\lambda_{v, 1}+\lambda_{v, 2}, v \mid \infty$. Let $\psi$ be the product of the central character of $\pi$ with $|\cdot|^{w_{\pi}}$, so that $\psi$ has finite order. Let $a$ denote the order of $\psi$, and let $U(2)_{a}$ denote the subgroup of $U(2)$ consisting of those matrices $g \in U(2)$ with $\operatorname{det}(g)^{a}=1$. Let $U(2)_{a} / \sim$ denote the set of conjugacy classes of $U(2)_{a}$. By "the Haar measure on 
$U(2)_{a} / \sim$ " we mean the push-forward of the Haar measure on $U(2)_{a}$ with total measure 1.

The Ramanujan conjecture is known to hold at all finite places of $\pi$ (see Bla06]), so for all $v$ for which $\pi_{v}$ is unramified, the matrix $(\mathbf{N} v)^{-w_{\pi} / 2} \operatorname{rec}\left(\pi_{v}\right)\left(\right.$ Frob $\left._{v}\right)$ lies in $U(2)_{a}$. Let $\left[\pi_{v}\right]$ denote its conjugacy class in $U(2)_{a} / \sim$.

Theorem 7.1.6. Suppose that $F$ is a totally real field and that $\pi$ is a non-CM regular algebraic cuspidal automorphic representation of $\mathrm{GL}_{2}\left(\mathbb{A}_{F}\right)$. Then the classes $\left[\pi_{v}\right]$ are equidistributed with respect to the Haar measure on $U(2)_{a} / \sim$.

Proof. This follows from Corollary 7.1.5, together with the corollary to Theorem I.A.2 of Ser68] (note that the irreducible representations of $U(2)_{a}$ are the representations $\operatorname{det}^{c} \otimes \operatorname{Sym}^{d} \mathbb{C}^{2}$ for $\left.0 \leq c<a, d \geq 0\right)$.

This may be reformulated in a somewhat more explicit fashion as follows. Note that the space $U(2)_{a} / \sim$ is disconnected if $a \neq 1$, so that to make an explicit statement we choose a connected component, which amounts to choosing $\zeta$ as in the statement below (of course, one may replace $\zeta$ by $-\zeta$, and it is only the choice of $\zeta^{2}$ which determines a component).

Corollary 7.1.7. Suppose that $F$ is a totally real field, and that $\pi$ is a non-CM regular algebraic cuspidal automorphic representation of $\mathrm{GL}_{2}\left(\mathbb{A}_{F}\right)$. Let $\psi$ be the product of the central character of $\pi$ with $|\cdot|^{w_{\pi}}$, so that $\psi$ is a finite order character. Let $\zeta$ be a root of unity with $\zeta^{2}$ in the image of $\psi$. For any place $v$ of $F$ such that $\pi_{v}$ is unramified, let $t_{v}$ denote the eigenvalue of the Hecke operator

$$
\left[\mathrm{GL}_{2}\left(\mathcal{O}_{F_{v}}\right)\left(\begin{array}{cc}
\varpi_{v} & 0 \\
0 & 1
\end{array}\right) \mathrm{GL}_{2}\left(\mathcal{O}_{F_{v}}\right)\right]
$$

(where $\varpi_{v}$ is a uniformiser of $\left.\mathcal{O}_{F_{v}}\right)$ on $\pi_{v}^{\mathrm{GL}_{2}\left(\mathcal{O}_{F_{v}}\right)}$. Note that if $\psi_{v}\left(\varpi_{v}\right)=\zeta^{2}$, then $t_{v} /\left(2(\mathbf{N} v)^{\left(1+w_{\pi}\right) / 2} \zeta\right) \in[-1,1] \subset \mathbb{R}$.

Then as $v$ ranges over the places of $F$ with $\pi_{v}$ unramified and $\psi_{v}\left(\varpi_{v}\right)=\zeta^{2}$, the values $t_{v} /\left(2(\mathbf{N} v)^{\left(1+w_{\pi}\right) / 2} \zeta\right)$ are equidistributed in $[-1,1]$ with respect to the measure $(2 / \pi) \sqrt{1-t^{2}} d t$

\section{ACKNOWLEDGMENTS}

The authors would like to thank Richard Taylor for some helpful discussions related to the content of this paper. They would also like to thank Florian Herzig and Sug Woo Shin for their helpful comments on an earlier draft.

\section{REFERENCES}

[AC89] James Arthur and Laurent Clozel, Simple algebras, base change, and the advanced theory of the trace formula, Annals of Mathematics Studies, vol. 120, Princeton University Press, Princeton, NJ, 1989. MR.1007299 (90m:22041)

[AT09] Emil Artin and John Tate, Class field theory, AMS Chelsea Publishing, Providence, RI, 2009, Reprinted with corrections from the 1967 original. MR2467155 (2009k:11001)

[Ber10] Laurent Berger, Représentations modulaires de $\mathrm{GL}_{2}\left(\mathbf{Q}_{p}\right)$ et représentations galoisiennes de dimension 2, Astérisque (2010), no. 330, 263-279. MR2642408

[Bla06] Don Blasius, Hilbert modular forms and the Ramanujan conjecture, Noncommutative geometry and number theory, Aspects Math., E37, Vieweg, Wiesbaden, 2006, pp. 3556. MR2327298 (2008g:11077) 
[BLGGT10] Tom Barnet-Lamb, Toby Gee, David Geraghty, and Richard Taylor, Potential automorphy and change of weight, preprint available at http://www. math.northwestern.edu/ gee/, 2010.

[BLGHT09] Tom Barnet-Lamb, David Geraghty, Michael Harris, and Richard Taylor, A family of Calabi-Yau varieties and potential automorphy II, Preprint, 2009.

[BW00] A. Borel and N. Wallach, Continuous cohomology, discrete subgroups, and representations of reductive groups, second ed., Mathematical Surveys and Monographs, vol. 67, American Mathematical Society, Providence, RI, 2000. MR1721403 (2000j:22015)

[CDT99] Brian Conrad, Fred Diamond, and Richard Taylor, Modularity of certain potentially Barsotti-Tate Galois representations, J. Amer. Math. Soc. 12 (1999), no. 2, 521-567. MR.1639612 (99i:11037)

[CHT08] Laurent Clozel, Michael Harris, and Richard Taylor, Automorphy for some l-adic lifts of automorphic mod $l$ Galois representations, Publ. Math. IHES 108 (2008), 1-181. MR 2470687 (2010j:11082)

[Clo82] Laurent Clozel, Changement de base pour les représentations tempérées des groupes réductifs réels, Ann. Sci. École Norm. Sup. (4) 15 (1982), no. 1, 45-115. MR672475 $(84 \mathrm{j}: 22015)$

[Dim05] Mladen Dimitrov, Galois representations modulo $p$ and cohomology of Hilbert modular varieties, Ann. Sci. École Norm. Sup. (4) 38 (2005), no. 4, 505-551. MR2172950 (2006k:11100)

[DKV84] P. Deligne, D. Kazhdan, and M.-F. Vignéras, Représentations des algèbres centrales simples p-adiques, Representations of reductive groups over a local field, Travaux en Cours, Hermann, Paris, 1984, pp. 33-117. MR771672 (86h:11044)

[Gee06] Toby Gee, A modularity lifting theorem for weight two Hilbert modular forms, Math. Res. Lett. 13 (2006), no. 5-6, 805-811. MR.2280776 (2007m:11065)

[Gee09] _ The Sato-Tate conjecture for modular forms of weight 3, Doc. Math. 14 (2009), 771-800. MR2578803

[Gee10] _ Automorphic lifts of prescribed types, to appear Math. Annalen, available at http://www.math.northwestern.edu/ gee/, 2010.

[Ger09] David Geraghty, Modularity lifting theorems for ordinary Galois representations, preprint, 2009.

[GG09] Toby Gee and David Geraghty, Companion forms for unitary and symplectic groups, Preprint, 2009

[Gue09] L. Guerberoff, Modularity lifting theorems for Galois representations of unitary type, Arxiv preprint arXiv:0906.4189 (2009).

[Har09] M. Harris, Potential automorphy of odd-dimensional symmetric powers of elliptic curves, and applications, Algebra, Arithmetic and Geometry-Manin Festschrift, Progress in Mathematics, vol. 270, Birkhäuser, Boston, MA, 2009. MR.2641185

[HSBT10] Michael Harris, Nick Shepherd-Barron, and Richard Taylor, A family of Calabi-Yau varieties and potential automorphy, Annals of Math. (2) 171 (2010), no. 2, 779-813. MR 2630056

[HT01] Michael Harris and Richard Taylor, The geometry and cohomology of some simple Shimura varieties, Annals of Mathematics Studies, vol. 151, Princeton University Press, Princeton, NJ, 2001, With an appendix by Vladimir G. Berkovich. MR.1876802 (2002m:11050)

[Kis07] Mark Kisin, Modularity of 2-dimensional Galois representations, Current developments in mathematics, 2005, Int. Press, Somerville, MA, 2007, pp. 191-230. MR 2459302

[Kis08] _ Potentially semi-stable deformation rings, J. Amer. Math. Soc. 21 (2008), no. 2, 513-546. MR2373358 (2009c:11194)

[Kis09] Moduli of finite flat group schemes, and modularity, Annals of Math. (2) 170 (2009), no. 3, 1085-1180.

[Lab09] Jean-Pierre Labesse, Changement de base CM et séries discrètes, preprint, 2009

[Lan89] R. P. Langlands, On the classification of irreducible representations of real algebraic groups, Representation theory and harmonic analysis on semisimple Lie groups, Math. Surveys Monogr., vol. 31, Amer. Math. Soc., Providence, RI, 1989, pp. 101170. MR:1011897 (91e:22017) 
[Sav05] David Savitt, On a conjecture of Conrad, Diamond, and Taylor, Duke Math. J. 128 (2005), no. 1, 141-197. MR.2137952 (2006c:11060)

[Ser68] Jean-Pierre Serre, Abelian l-adic representations and elliptic curves, McGill University lecture notes written with the collaboration of Willem Kuyk and John Labute, W. A. Benjamin, Inc., New York-Amsterdam, 1968. MR 0263823(41:8422)

[Ser94] Sur la semi-simplicité des produits tensoriels de représentations de groupes, Invent. Math. 116 (1994), no. 1-3, 513-530. MR1253203 (94m:20091)

[Tay06] Richard Taylor, On the meromorphic continuation of degree two L-functions, Doc. Math. (2006), no. Extra Vol., 729-779 (electronic). MR.2290604(2008c:11154)

[Tay08] - Automorphy for some l-adic lifts of automorphic mod l Galois representations. II, Publ. Math. IHES 108 (2008), 183-239. MR2470688(2010j:11085)

[TY07] Richard Taylor and Teruyoshi Yoshida, Compatibility of local and global Langlands correspondences, J. Amer. Math. Soc. 20 (2007), no. 2, 467-493 (electronic). MR 2276777 (2007k:11193)

[Zel80] A. V. Zelevinsky, Induced representations of reductive $\mathfrak{p}$-adic groups. II. On irreducible representations of GL(n), Ann. Sci. École Norm. Sup. (4) 13 (1980), no. 2, 165-210. MR.584084 (83g:22012)

Department of Mathematics, Brandeis University, 415 South Street MS 050, Waltham, MASSACHUSETTS 02138

E-mail address: tbl@brandeis.edu

Department of Mathematics, Harvard University, Cambridge, Massachusetts 02138

E-mail address: tgee@math.harvard.edu

Current address: Department of Mathematics, Northwestern University, 2033 Sheridan Road Evanston, Ilinois 60208-2730

E-mail address: gee@math.northwestern.edu

Department of Mathematics, Harvard University, Cambridge, Massachusetts 02138

E-mail address: geraghty@math.harvard.edu

Current address: Princeton University and Institute for Advanced Study, Princeton, New Jersey 08540

E-mail address: geraghty@math.ias.edu 